Portland State University

PDXScholar

$1-1-2010$

\title{
Numerical Modeling of Electromagnetic Scattering in Explosive Granular Media
}

Garth Sundberg

Portland State University

Follow this and additional works at: https://pdxscholar.library.pdx.edu/open_access_etds Let us know how access to this document benefits you.

\section{Recommended Citation}

Sundberg, Garth, "Numerical Modeling of Electromagnetic Scattering in Explosive Granular Media" (2010). Dissertations and Theses. Paper 865.

https://doi.org/10.15760/etd.865

This Dissertation is brought to you for free and open access. It has been accepted for inclusion in Dissertations and Theses by an authorized administrator of PDXScholar. Please contact us if we can make this document more accessible: pdxscholar@pdx.edu. 
Numerical Modeling of Electromagnetic Scattering in Explosive Granular Media

by

Garth Sundberg

A dissertation submitted in partial fulfillment of the requirements for the degree of

Doctor of Philosophy

in

Electrical and Computer Engineering

Dissertation Committee:

Lisa M. Zurk, Chair

Richard Campbell

Edward Godshalk

Melinda Holtzman

Branimir Pejcinovic

Erik Sanchez

Portland State University

(C) 2010 


\section{ABSTRACT}

Terahertz $(\mathrm{THz})$ reflection and transmission spectroscopy is a promising new field with applications in imaging and illicit material detection. One particularly useful application is for the detection of improvised explosive devices (IEDs) which is a favorite weapon of global terrorists. Explosive materials have been shown to have a unique spectral signature in the $\mathrm{THz}$ band which can be used to identify the explosives. However, the initial measurements performed on the explosive samples do not account for the modulation of the spectral features by random scattering that will be prevalent with actual samples encountered in applications.

The intent of this work is to characterize and quantify the effects of random scattering that may alter the spectral features. Specifically, the effect that a randomly rough surface and granular scattering has on the scattered $\mathrm{THz}$ wave (T-Rays) will be investigated and characterized using the Finite-Difference TimeDomain (FDTD) simulation method. The FDTD method is a natural choice for this work as it can handle complicated geometries (i.e., multiple scatterers, arbitrarily rough interfaces, etc.) arbitrary materials (i.e., dispersive media, etc.) and provides broadband frequency data with one simulation pass. 
First, the effect that the randomly rough surface of the sample explosive has on the extracted spectral signature will be studied using a Monte-Carlo analysis. Then the effect of the complex structure inside the explosive material (the granular scatterers) will be considered. Next, when the physics of the rough surface and granular scattering are understood, a robust method to extract the spectral signature from the reflected T-rays will be developed. 


\section{Acknowledgments}

I would like to thank my advising Professor, Dr. Lisa M. Zurk, for providing me the opportunity to work with her on such an interesting topic. In addition, her guidance and thoughtful comments as I completed this journey were invaluable.

Thank you to Dr. John Schneider of Washington State University. Dr. Schneider's discussion on FDTD simulations, NTFF transformations, AFP formulations and simulating dispersive media was invaluable.

I would like to thank my friend Dr. Edward Godshalk for his support and understanding while I pursued this degree. I would also like to thank Ed for his enthusiastic suggestion to start this degree.

Thanks are due to Portland State University Professor Dr. Branimir Pejcinovic for getting me started in the Ph.D. program at Portland State University.

I would like to thank Dr. Richard Campbell for his advice on how to survive the Ph.D. graduate experience.

Thanks to Dr. Richard Lutz for his discussion of electromagnetic theory, and on the finer points of using the software needed to complete this degree.

Thank you to Mr. Vijay Ullal and the management at Maxim Integrated 
Products for paying for the tuition required to complete this degree.

Thank you to Drs. Lisa M. Zurk, Richard Campbell, Branimir Pejcinovic, and Melinda Holtzman of the Electrical Engineering Department at Portland State University for serving on my Committee. Thank you to Dr. Edward Godshalk of Maxim Integrated Products for serving on my Committee. Thank you to Dr. Erik Sanchez of the Physics Department at Portland State University for serving on my Committee as the Graduate Representative.

Finally, a special thanks to my wife, Jennifer, and daughters, Valerie and Vivian, for their support. Without Jennifer's insistence and support, I would never have started, or completed, this degree. Jennifer, I owe this all to you. 


\section{Contents}

Acknowledgments

List of Tables $\quad$ x

List of Figures xiii

1 Introduction 1

1.1 Focus of Dissertation . . . . . . . . . . . . . . 5

1.2 Summary of Dissertation . . . . . . . . . . . . . . 8

2 Electromagnetic Scattering Theory 11

2.1 Components of Explosives . . . . . . . . . . . . . . 11

2.2 Terahertz Measurement System . . . . . . . . . . . . . . 13

2.3 Fresnel Reflection and Transmission Coefficients . . . . . . . . . . 16

2.3.1 Normal Incidence . . . . . . . . . . . . . . . . 16

2.3.2 Oblique Incidence . . . . . . . . . . . . . . . . . 18

2.4 Rough Surface Scattering . . . . . . . . . . . . . . . . . . 20

2.4.1 One-dimensional Gaussian Random Rough Surface . . . . 21 
2.4.2 Rough Surface Scattering Fundamentals . . . . . . . . . . 22

2.4.3 Calculating Rough Surface Scattering . . . . . . . . . 25

2.5 Granular Scattering . . . . . . . . . . . . . . . . 30

2.5.1 Quasi-Crystalline Approximation ........... 31

2.5.2 Born Approximation . . . . . . . . . . . . . . 32

2.5.3 Rytov Approximation .............. 32

2.5.4 Numerical Methods . . . . . . . . . . . . . . . . 32

2.6 Selection of Modeling Technique . . . . . . . . . . . . . 33

2.7 Conclusion . . . . . . . . . . . . . . . . . . . . . . . . 34

3 The Finite-Difference Time-Domain Method 36

3.1 Yee Algorithm . . . . . . . . . . . . . . . . . . 37

3.1.1 Maxwell's Equations . . . . . . . . . . . . . . 38

3.1.2 Finite-Difference Notation . . . . . . . . . . . . . . 42

3.1.3 Maxwell's Equations in Finite-Difference Notation . . . . . 43

3.2 FDTD Sources: The Total-Field/Scattered-Field Method . . . . . 47

3.2.1 Analytic Field Propagation Method . . . . . . . . . . . 54

3.3 Grid Truncation: The Perfectly Matched Layer . . . . . . . . . . 59

3.3.1 Implementation of UPML in FDTD . . . . . . . . . . . 62

3.4 Near-to-Far-Field Transformation . . . . . . . . . . . . . . . . 70

3.5 Dispersive Media . . . . . . . . . . . . . . . . . . . . 79 
3.5.1 Lorentz Media . . . . . . . . . . . . . . . . . . . . . 80

3.5.2 Modify the FDTD update equations for the Lorentz Model. 82

3.6 Validation of FDTD Calculation . . . . . . . . . . . 86

3.6.1 The Basic FDTD Simulation Routine . . . . . . . . . . 87

3.6.2 AFP Implementation of the TFSF Method . . . . . . . 88

3.6.3 Implementation of UPML $\ldots \ldots \ldots \ldots$. . . . . . . . 92

3.6.4 Implementation of the NTFF transform $\ldots \ldots \ldots$. . . 93

3.6.5 Implementation of the Lorentz model for dispersive media 96

3.7 Conclusion . . . . . . . . . . . . . . . . . . . . . 98

4 Terahertz Measurements 100

4.1 Terahertz Transmission Measurement and Model Results . . . . . 100

4.2 Terahertz Reflection Measurements and Model Results . . . . . . 104

4.3 Conclusion . . . . . . . . . . . . . . . . . . . . . . 108

5 Effect of Random Media Interface on Composition-4 Spectral

$\begin{array}{ll}\text { Signature } & 110\end{array}$

5.1 Monitoring Spectral Peaks . . . . . . . . . . . . . . . . 111

5.2 Impact of Rough Surface on Composition-4 Spectral Signature . . 113

5.3 Impact of Surface Samples on Composition-4 Spectral Signature . 119

5.4 Impact of Multiple Surface Samples on Composition-4 Spectral

Signature . . . . . . . . . . . . . . . . 122 
5.4.1 Rougher Surface . . . . . . . . . . . . . . . . 123

5.4 .2 Smoother Surface . . . . . . . . . . . . . . 127

5.5 Impact of System Noise . . . . . . . . . . . . . . . . . 130

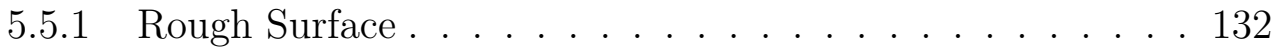

5.5.2 Smoother Surface . . . . . . . . . . . . . . . . 133

5.6 Changing the Angle of Incidence . . . . . . . . . . . . . . . 135

5.6.1 Rough Surface Variation With Angle of Incidence . . . . . 136

5.6.2 Smoother Surface Variation With Angle of Incidence . . . 137

5.7 Conclusion . . . . . . . . . . . . . . . . . . . . . . 139

6 Effect of Granular Scattering $\quad 140$

6.1 Modeling the Granular Nature of Composition-4 . . . . . . . . . . 140

6.2 Impact of the Granular Nature of Composition-4 Spectral Signature: No System Noise . . . . . . . . . . . . . . . . . 143

6.2.1 Small Grain Size . . . . . . . . . . . . . . . . 143

6.2.2 Larger Grain Size . . . . . . . . . . . . . . . . . . . . . . 144

6.3 Impact of the Granular Nature of Composition-4 Spectral Signature: With System Noise . . . . . . . . . . . . . . . . . 146

6.3.1 Small Grain Size . . . . . . . . . . . . . 146

6.3.2 Larger Grain Size . . . . . . . . . . . . . . . . . . . 148

6.4 Other Angles of Incidence . . . . . . . . . . . . . . . . . . 149 
Conclusion ..................... . . 149

7 Combined Effect of Random Media Interface and Granular Scattering

7.1 Modeling the Rough Surface and Granular Nature of Composition-4151

7.2 Impact of the Rough Surface and the Granular Nature of Composition-4 Spectral Signature: No System Noise . . . . . . . . 153

7.3 Impact of the Rough Surface and the Granular Nature of Composition-4 Spectral Signature: With System Noise . . . . . . 156

7.4 Other Angles of Incidence . . . . . . . . . . . . . . . . . . . 158

7.4.1 Rough Surface with Small Grain Size . . . . . . . . . 158

7.4.2 Rough Surface with Large Grain Size . . . . . . . . . . . . 159

7.4.3 Smoother Surface with Small Grain Size . . . . . . . . . . 160

7.4.4 Smoother Surface with Larger Grain Size . . . . . . . . . . 162

7.5 Conclusion . . . . . . . . . . . . . . . 163

8 Conclusion and Future Work 164

8.1 Conclusion . . . . . . . . . . . . . . . . . . . 164

8.2 Future Work . . . . . . . . . . . . . . . . . 166

$\begin{array}{ll}\text { References } & 168\end{array}$

$\begin{array}{ll}\text { Appendix A Glossary } & 180\end{array}$ 


\section{List of Tables}

2.1 Dielectric Constants of Explosive Grains. . . . . . . . . . . . . . . 13

2.2 Common mixtures of explosives in percentages from Meyer [22]. . 14

2.3 Location of Absorption Peaks for Explosive Crystals. . . . . . . . 16

3.1 Lorentz model parameters for C-4 [10]. . . . . . . . . . . . . . . 81

3.2 Rough Surfaces simulated for NTFF validation. Note, $\lambda$ is for free-space. . . . . . . . . . . . . . . . . . 96

4.1 Values of RMS height $\left(h_{r m s}\right)$ and correlation length $\left(\mathrm{L}_{C}\right)$ used in the scattering calculations for the sandpaper samples [60]. . . . 105

5.1 Values of RMS height $\left(h_{r m s}\right)$ and correlation length $\left(\mathrm{L}_{C}\right)$ used in the scattering calculations for the sandpaper samples [60]. . . . 114

5.2 Lorentz poles with a $\pm 5 \%$ range for C-4 [10]. . . . . . . . . . . 117 
5.3 Location (in $\mathrm{THz}$ ) of extracted Lorentz poles for data sampled in the backscatter direction from a homogenous C-4 sample with the surface roughness of 36 grit sandpaper. Data reported for peaks within $5 \%$ of the expected value. . . . . . . . . . . . 137

5.4 Location (in $\mathrm{THz}$ ) of extracted Lorentz poles for data sampled in the backscatter direction from a homogenous C-4 sample with the surface roughness of 80 grit sandpaper. Data reported for peaks within $5 \%$ of the expected value. Red values are within $10 \%$. . . 138

6.1 Simulation cases run to study granular scattering. . . . . . . . . 141

7.1 Simulation cases run to study granular scattering. . . . . . . . . 152

7.2 Location (in $\mathrm{THz}$ ) of extracted Lorentz poles for data sampled at $-10^{\circ},-30^{\circ}$, and $-50^{\circ}$ from a background of C-4 with the surface roughness of 36 grit sandpaper and a $100 \mu \mathrm{m}$ grain size. Data reported for peaks within $5 \%$ of the expected value. Red values are within $10 \% \ldots \ldots \ldots \ldots \ldots \ldots$

7.3 Location (in $\mathrm{THz}$ ) of extracted Lorentz poles for data sampled at $-10^{\circ},-30^{\circ}$, and $-50^{\circ}$ from a background of C-4 with the surface roughness of 36 grit sandpaper and a $300 \mu \mathrm{m}$ grain size. Data reported for peaks within $5 \%$ of the expected value. . . . . . 160 
7.4 Location (in $\mathrm{THz}$ ) of extracted Lorentz poles for data sampled at $-10^{\circ},-30^{\circ}$, and $-50^{\circ}$ from a background of C-4 with the surface roughness of 80 grit sandpaper and a $100 \mu \mathrm{m}$ grain size. Data reported for peaks within $5 \%$ of the expected value. Red values are within $10 \% \ldots \ldots \ldots \ldots \ldots \ldots \ldots$

7.5 Location (in THz) of extracted Lorentz poles for data sampled at $-10^{\circ},-30^{\circ}$, and $-50^{\circ}$ from a background of $\mathrm{C}-4$ with the surface roughness of 80 grit sandpaper and a $100 \mu \mathrm{m}$ grain size. Data reported for peaks within $5 \%$ of the expected value. Red values

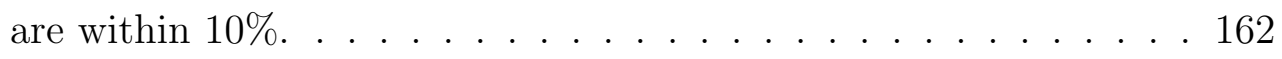




\section{List of Figures}

1.1 Sample absorption peaks from Burnett et. al.[9]. . . . . . . . . . 3

1.2 Sample of granular structure in the common explosive HMX from Reaugh $[19] \ldots \ldots \ldots \ldots \ldots$

2.1 Photograph of T-Ray 4000 with transmit and receive heads. . . .

2.2 A THz pulse propagating in air measured with a Picometrix TRay 4000. Pulse duration is approximately 5 ps. Inset is the power spectral density (PSD) of the pulse. The usable portion of the spectrum spans from $0.1 \mathrm{THz}$ to $2 \mathrm{THz} . \ldots \ldots \ldots$

2.3 Normal incidence for either parallel or perpendicular polarization. 17

2.4 Oblique incidence for either parallel (E-field polarization parallel to the plane) or perpendicular polarization (E-field polarization perpendicular to the plane). Perpendicular polarization is illustrated in this figure. . . . . . . . . . . . . . . . . . 19

2.5 One-dimensional random rough surface $\ldots \ldots \ldots$

2.6 A sample of an infinitely wide smooth surface metal interface. . . 23 
2.7 Smooth surface scattering for an incident wave at $30^{\circ}$ from the normal. All of the energy is reflected at $30^{\circ} \ldots \ldots \ldots$

2.8 A rough surface metal interface with $\mathrm{h}_{r m s}=0.05 \lambda$ and $\mathrm{L}_{C}=0.2 \lambda$

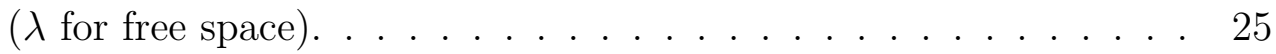

2.9 Rough surface scattering $\left(\mathrm{h}_{r m s}=0.05 \lambda\right.$ and $\mathrm{L}_{C}=0.2 \lambda, \lambda$ for free space) for an incident wave at $30^{\circ}$ from the normal. Most of the energy is reflected at $30^{\circ} \ldots \ldots \ldots \ldots \ldots \ldots \ldots$

2.10 A rough surface metal interfacewith $\mathrm{h}_{r m s}=0.2 \lambda$ and $\mathrm{L}_{C}=1.5 \lambda$ $(\lambda$ for free space) $\ldots \ldots \ldots \ldots \ldots \ldots \ldots$

2.11 Rough surface scattering $\left(\mathrm{h}_{r m s}=0.2 \lambda\right.$ and $\mathrm{L}_{C}=1.5 \lambda, \lambda$ for free space) for an incident wave at $30^{\circ}$ from the normal. More energy is spread over a larger observation angle. . . . . . . . . . . . 27

2.12 Sample of background material with granular scatterers. . . . . . 33

3.1 Position of the electric and magnetic field vector components about a cubic unit cell. . . . . . . . . . . . . . . . . . 37

3.2 A one dimensional FDTD grid illustrating the leapfrog arrangement. 42

3.3 Showing a FDTD grid with a total field region and scattered field region $[34] \ldots \ldots \ldots \ldots \ldots$

3.4 The FDTD grid showing each field point and the TFSF boundary $[39] . \ldots \ldots \ldots \ldots \ldots \ldots$ 
3.5 The FDTD grid showing where the absorbing PML is located. . . 60

3.6 Showing a FDTD grid with a total field region and scattered field region. .......................... 72

3.7 Far-Field observation point relative to the near-field integration boundary. ......................... 74

3.8 Integration contour, $C_{i}$ showing the $H$ field offset from the contour $C_{i} \ldots \ldots \ldots \ldots \ldots 78$

3.9 Integration contour for a one-dimensional rough surface. . . . . . 78

3.10 The complex frequency dependent dielectric constant of C-4. The left axis is the real part and the right axis is the imaginary part. . 82

3.11 Time-domain representation of a ricker wavelet centered at $3 \mathrm{THz}$. Inset is the frequency content of the ricker wavelet. . . . . . . . 88

3.12 Ricker wavelet being introduced into, and propagating through, the FDTD grid using a hard source. . . . . . . . . . . . . . . 89

3.13 The plane-wave as it moves through the FDTD grid. The top material is plasitc $\epsilon_{r}=4$ and the bottom material is air $\epsilon_{r}=1$. . 91

3.14 Amount of leaked fields at "X" in Figure 3.13 . . . . . . . . . . . 92

3.15 Ricker wavelet propagating through the FDTD grid with a UPML. The wave leaves the grid with minimal reflection. . . . . . . . . . 94

3.16 E-field over all time of the FDTD simulation sampled at "X" in Figure 3.15 on the PML interface. . . . . . . . . . . . . 95 
3.17 E-field over all time of the FDTD simulation sampled at "X" in

Figure 3.15 with no PML interface. . . . . . . . . . . . . . . . 95

3.18 Bistatic scattering for a rough metal surface with $\mathrm{rms}$ height $=0.05 \lambda$ and a correlation length $=0.2 \lambda . \lambda$ is for free space. . . . . . . . 96

3.19 Bistatic scattering for a rough metal surface with $\mathrm{rms}$ height $=0.05 \lambda$ and a correlation length $=0.35 \lambda . \quad \lambda$ is for free space. . . . . . . . 97

3.20 Bistatic scattering for a rough metal surface with $\mathrm{rms}$ height $=0.2 \lambda$ and a correlation length $=1.5 \lambda . \quad \lambda$ is for free space. . . . . . . . 97

3.21 The extracted real part of the permittivity vs. the modeled real part of the permittivity. . . . . . . . . . . . . . . . 98

3.22 Percent difference in the extracted vs. modeled real part of C-4 permittivity. . . . . . . . . . . . . . . . . . . 99

4.1 Picture of small grain polyethylene (SGPE) on the left and large grain polyethylene (LGPE) on the right from the UMBC $\mathrm{THz}$ transmission experiment. . . . . . . . . . . . . 101

4.2 Experimental set-up for $\mathrm{THz}$ spectroscopy at UMBC. . . . . . . . 102

4.3 FDTD model space for the UMBC scattering simulations. Transmitted field shows coherent (solid line) and incoherent (dashed line) fields. . . . . . . . . . . . . . . . . . . 103 
4.4 Measured (dashed line) vs. modeled (dashed line with circles) response of transmission spectroscopy through PE sample. LGPE data truncated when the measured data reaches the noise floor. The reference curve is shown in blue and the samples are shown in red. . . . . . . . . . . . . . . . . . . . . . 104

4.5 Optical picture of gold-coated sandpaper. P80 is the 80 grit sandpaper and P36 is the 36 grit sandpaper. . . . . . . . . 105

4.6 Schematic illustration of measurement setup. . . . . . . . . 106

4.7 Coherent reflected power, relative to a perfectly reflecting surface, for the 36 and 80 grit sandpaper. The measure data is shown without symbols. Monte Carlo FDTD and KA results are lines with symbols. . . . . . . . . . . . . . . . . . . 107

4.8 Power relative to a perfectly reflecting surface for 36,80 , and 120 grit sandpaper. The mean for each sample is shown in black. . . . 109

5.1 Comparison of the reflected power between FDTD results and calculated results for a wave of normal incidence. . . . . . . . . . . 112

5.2 Comparison of the derivative of the reflected power between FDTD results and calculated results for a wave of normal incidence. The location of the peaks is the same as that listed in Table 3.1. . . 112

5.3 Schematic representation of rough surface simulations. . . . . 113 
5.4 Representative RCS at $0.5 \mathrm{THz}$ of homogeneous C-4 with a surface roughness of 36 and 80 grit sandpaper. Angle of incident plane wave is $-37^{\circ}$ measured from the surface normal. Fifty surfaces are averaged. . . . . . . . . . . . . . . . . . . . . . . . . 114

5.5 FDTD simulated RCS data of homogeneous C-4 sampled over frequency in the backscatter direction for 36 grit sandpaper. Fifty surfaces are averaged. . . . . . . . . . . . . . . . . . . 115

5.6 Derivative of smoothed data sampled from the FDTD RCS data sampled in the backscatter direction from a homogeneous C-4 sample with the surface roughness listed in Table 5.1. Fifty samples were averaged. The arrows correspond to the location (within 5\%) of the Lorentz poles from Table 5.2. . . . . . . . . . . . . . . . 118

5.7 Derivative of smoothed data sampled from the FDTD RCS data sampled in the backscatter direction from a homogeneous C-4 sample with a 36 grit surface roughness listed in Table 5.1. Fifty samples were averaged. Width of the Gaussian filter is swept from $1 / 12^{\text {th }}$ to $1 / 24^{\text {th }}$ of the sample space. . . . . . . . . . . 120 
5.8 Derivative of smoothed data sampled from the FDTD RCS data sampled in the backscatter direction from a homogeneous C-4 sample with the surface roughness listed in Table 5.1. Ten samples were averaged. The arrows correspond to the location (within $5 \%$ ) of the Lorentz poles from Table 5.2. . . . . . . . . . . . 121

5.9 Derivative of smoothed data sampled at $-37^{\circ}$ from a homogeneous C-4 sample with 36 grit surface roughness. First five sets of twenty samples are averaged. The black and red arrows identify the peaks in Table 5.2 to within $5 \%$ and $10 \%$ respectively. . . . . . . . . 124

5.10 Derivative of smoothed data sampled at $-37^{\circ}$ from a homogeneous C-4 sample with 36 grit surface roughness. Second five sets of twenty samples are averaged. The black and red arrows identify the peaks in Table 5.2 to within $5 \%$ and $10 \%$ respectively. . . . 125

5.11 Derivative of smoothed data sampled at $-37^{\circ}$ from a homogeneous C-4 sample with 36 grit surface roughness. Fifty samples are averaged. The black and red arrows identify the peaks in Table 5.2 to within $5 \%$ and $10 \%$ respectively. . . . . . . . . 126

5.12 Derivative of smoothed data sampled at $-37^{\circ}$ from a homogeneous C-4 sample with 80 grit surface roughness. First five sets of twenty samples are averaged. The black and red arrows identify the peaks in Table 5.2 to within $5 \%$ and $10 \%$ respectively. . . . . . . . 128 
5.13 Derivative of smoothed data sampled at $-37^{\circ}$ from a homogeneous C-4 sample with 80 grit surface roughness. Second five sets of twenty samples are averaged. The black and red arrows identify the peaks in Table 5.2 to within $5 \%$ and $10 \%$ respectively. . . . . 129

5.14 Derivative of smoothed data sampled at $-37^{\circ}$ from a homogeneous C-4 sample with 80 grit surface roughness. Fifty samples are averaged. The black and red arrows identify the peaks in Table 5.2 to within $5 \%$ and $10 \%$ respectively. . . . . . . . . . . 131

5.15 Derivative of smoothed data sampled at $-37^{\circ}$ from a homogeneous C-4 sample with 36 grit surface roughness. Fifty samples were averaged. Noise was added to get an SNR of 10dB and 20dB. . . 133

5.16 Derivative of smoothed data sampled at $-37^{\circ}$ from a homogeneous C-4 sample with 80 grit surface roughness. Fifty samples were averaged. Noise was added to get an SNR of 10dB and 20dB. . . 134

6.1 Schematic representation of granular simulations. . . . . . . . . . 141

6.2 Derivative of smoothed data sampled at $-37^{\circ}$ from a background of C-4 with a smooth interface and a grain size of $100 \mu \mathrm{m}$. Fifty samples were averaged. The black arrows identify peaks in Table 5.2.

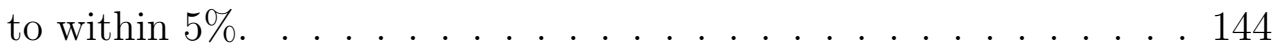


6.3 Derivative of smoothed data sampled at $-37^{\circ}$ from a background of C-4 with a smooth interface and a grain size of $300 \mu \mathrm{m}$. Fifty samples were averaged. The black arrows identify peaks in Table 5.2. to within $5 \% \ldots \ldots \ldots \ldots \ldots \ldots \ldots \ldots$

6.4 Derivative of smoothed data sampled at $-37^{\circ}$ from a background of C-4 with a smooth interface and a grain size of $100 \mu \mathrm{m}$. Fifty samples were averaged. Noise was added to get an SNR of 10dB

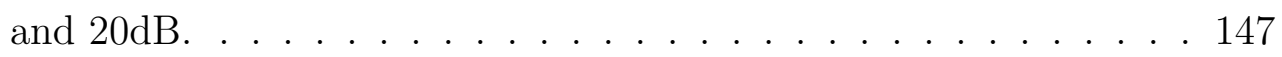

6.5 Derivative of smoothed data sampled at $-37^{\circ}$ from a background of C-4 with a smooth interface and a grain size of $300 \mu \mathrm{m}$. Fifty samples were averaged. Noise was added to get an SNR of $10 \mathrm{~dB}$ and $20 \mathrm{~dB} \ldots \ldots \ldots \ldots \ldots \ldots$

7.1 Derivative of smoothed data sampled at $-37^{\circ}$ from a background of C-4 with a 36 grit surface and a grain size of $100 \mu \mathrm{m}$. Fifty samples were averaged. The black arrows identify the peaks in Table 5.2 to within $5 \%$. . . . . . . . . . . . . . . . . . 154

7.2 Derivative of smoothed data sampled at $-37^{\circ}$ from a background of C-4 with a 80 grit surface and a grain size of $100 \mu \mathrm{m}$. Fifty samples were averaged. The black arrows identify the peaks in Table 5.2 to within $5 \% \ldots \ldots \ldots \ldots \ldots \ldots \ldots$ 
7.3 Derivative of smoothed data, with various SNR, sampled at $-37^{\circ}$

from a background of C-4 with a 36 grit interface and a grain size

of $100 \mu \mathrm{m}$. Fifty samples were averaged. . . . . . . . . . . 157 


\section{Chapter 1}

\section{Introduction}

The recent advances of ultrafast optical lasers have improved the generation and detection of energy in the terahertz $(\mathrm{THz})$ portion of the electromagnetic (EM) spectrum. Two promising areas for this newly opened portion of the EM spectrum are imaging and illicit material detection. Research is being actively pursued in $\mathrm{THz}$ time-domain spectroscopy (THz-TDS) to get broadband $\mathrm{THz}$ pulses for sample characterization. THz-TDS systems are being used for medical imaging [1], chemical detection $[2,3,4]$ and explosives detection $[2,4,5,6,7]$. The advantages of using $\mathrm{THz}$ waves for imaging and illicit material detection include [4]:

1. $\mathrm{THz}$ radiation is readily transmitted through most non-polar media. This enables THz systems to "see through" packaging, clothing, shoes, etc. to probe for dangerous materials. However, because of water absorbtion, the range of $\mathrm{THz}$ waves in air is limited. 
2. Many materials of interest for security applications (including explosives) have unique characteristics in the $\mathrm{THz}$ spectrum to allow for detection.

3. $\mathrm{THz}$ radiation poses either no, or minimal, health risk to either a suspect being scanned, or the system operator.

For the case of explosives, current news events detail the use of many different types of improvised explosive devices (IEDs) by global terrorists. These IEDs, while typically found in war zones, can be hidden anywhere. Because of the increasing skill of the terrorists to hide IEDs, the need for fast, accurate and reliable detection of explosive devices is readily apparent. THz-TDS has recently gained significant attention for its ability to detect explosives.

The THz-TDS technique works because of a lattice vibration when the explosive material is struck with the $\mathrm{THz}$ wave[8]. When a $\mathrm{THz}$ wave illuminates an explosive sample, the electrons gain enough energy to move from one quantized energy state to another quantized energy state. This transition shows up as absorption peaks in the THz spectral response (Figure 1.1) [9]. These absorption peaks appear at unique frequencies, thus allowing for the identification of the specimen illuminated (i.e., each specimen has a unique spectra or "fingerprint"). Because the spectral peaks of the explosive materials are unique to the $\mathrm{THz}$ frequency band, they do not appear during optical imaging. Also, the THz radiation does not ionize the illuminated specimen. This allows a $\mathrm{THz}$ detection 


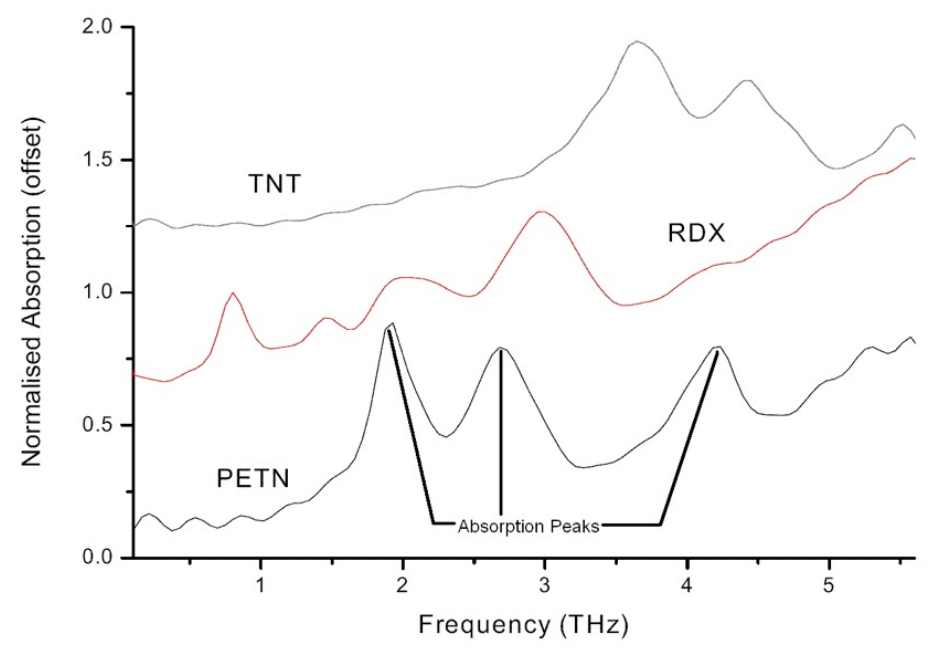

Figure 1.1: Sample absorption peaks from Burnett et. al.[9].

system to be safely used for the scanning of humans without fear of damage or injury.

There are methods currently available for explosive detection, including the use of ion mobility, x-rays, and metal detectors [10]. However, these current detection methods do not allow for the scanning of carefully packaged explosives, or explosive devices that do not contain metal. Beyond these limitations, the use of x-rays would not be a practical, or safe, method to scan humans because of the damage the x-rays may cause [10].

Most of the work on using $\mathrm{THz}$ radiation for the detection of explosives has focused on the production of $\mathrm{THz}$ sources and detectors or making measurements with these systems. Some have looked at the temperature dependence of 
the spectral peaks [5]. Others have looked at the THz systems for general measurements $[1,11]$. Several have looked at the use of $\mathrm{THz}$ waves specifically for the characterization of explosives $[2,4,6,7,11,12]$. Still others have used $\mathrm{THz}$ spectroscopy for the detection of biological agents or illicit drugs in addition to characterizing explosives $[3,4,11]$. In these papers, the measured samples were prepared for characterization (i.e., well defined samples with smooth surfaces). The prior work does not address some of the problems that will occur in an actual detection system (e.g rough surface scattering, granular scattering, etc.). Recently, some work has been done to investigate the effect that the rough surface has on the scattered wave $[13,14,15]$. This work was mainly restricted to rough surface scattering from metal surfaces or the effect a rough surface has on the Fresnel transmission coefficient.

However, a critical limitation to standoff sensing is the high sensitivity in the $\mathrm{THz}$ band to atmospheric water content that will likely limit current remote sensing applications to a distance of 100 meters at most. Application of $\mathrm{THz}$ sensing at longer distances may be possible by selecting "windows" between the water absorption bands [16]. In fact, Zhong et. al. have shown the detection of the $0.82 \mathrm{THz}$ peak (or "fingerprint") of the explosive material RDX can be detected from a distance of 30 meters utilizing a non-lossy window in the air absorption spectrum [17]. Additionally, a recent technology development is to propagate energy in another less attenuating band (e.g. using lasers), and use 
a nonlinear conversion method to locally produce $\mathrm{THz}$ energy in the vicinity of the target or area of interest [18].

\subsection{Focus of Dissertation}

The work that is the topic of this dissertation is to determine what effect the rough surface of the explosive will have on the spectral response. When a plane wave reflects off of a perfectly smooth surface, energy is perfectly phase coherent and only in the specular direction (i.e., the reflected angle equals the incident angle as predicted by the law of reflection). As the surface is made rough, the energy from the specular direction will be distributed into other directions reducing the coherent reflection and increasing the diffuse, incoherent reflection. As the surface becomes increasingly rough, the specular scattering will be lost to the diffuse scattering. The effect that the incoherent scattering has on the spectral fingerprint of common explosives must be understood if THz-TDS is to become a viable method for the detection of explosives.

Additional work that must be done is understanding the impact of granular scattering. Figure 1.2 shows a picture of the structure of the common explosive HMX [19]. The size of the particles in the explosive are on the same order as the wavelength of the $\mathrm{THz}$ waves being used to detect the explosives. Since the particles are on the same order as the wavelength of the $\mathrm{THz}$ radiation, there will be significant scattering in the Mie regime (i.e. the particle radius is approximately 


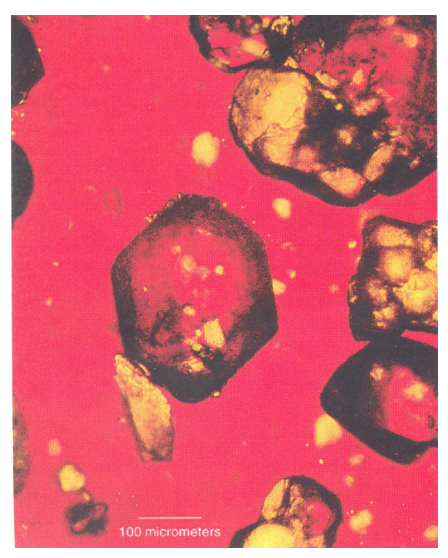

Figure 1.2: Sample of granular structure in the common explosive HMX from Reaugh [19].

the same size as the wavelength) [20]. What impact this Mie scattering will have on the spectral fingerprint is unknown and must be determined if THz-TDS is to become a viable method for the detection of explosives.

The random nature of the explosives (both the rough surface and the distribution of the particles shown in Figure 1.2) may require some intelligence to deal with the effect of the random scattering. Once the random scattering is understood, a more robust sensing system, a more robust incident wave, or signal processing may be developed to reduce the random scattering effects.

To study and understand the random scattering effects listed above (i.e., rough surface and granular scattering) a modeling method must be selected. The rough surface and granular scattering would be nearly impossible to analyze with closed form expressions by hand. Therefore, a numerical technique is best suited for the solution of the scattering from random media. The numerical technique 
selected is the the Finite-Difference Time-Domain (FDTD) [21] because of its versatility. To develop the FDTD simulator, the following was completed:

1. Programming the FDTD algorithm for wave propagation.

2. Adding a Perfectly Matched Layer (PML) Absorbing Boundary Condition $(\mathrm{ABC})$ at the edge of the simulation space to reduce spurious reflections.

3. Implementing the Analytic Field Propagation (AFP) method to source a plane wave against a non-dispersive half-plane interface to simulate the rough surface scattering from an explosive.

4. Altering the FDTD algorithm to handle dispersive material.

5. Adding a near-field to far-field transform to convert the near-field FDTD simulation results to far-field quantities.

6. Using the FDTD method to model the random nature of the media, which is unique to this work.

While the creation of a modeling tool is required for this work, the steps listed above can be accomplished by reading the literature on FDTD simulation techniques. What is new to this work is:

1. As the FDTD method is a classical electromagnetic solution, it will not directly model the vibrational resonance effects which cause the unique 
THz spectral signature. To simulate the vibrational effect, the explosive material will be modeled as a dispersive medium using the Lorentz model which captures the changing loss with frequency due to the lattice vibrations. This will allow the FDTD classical simulation model to predict the the frequency dependent reflections caused by quantum effects from the explosive material.

2. Modify the AFP method to work with a dispersive media. The literature for the AFP method does not include any work with dispersive media.

3. A new method of extracting the spectral signature from the random surface and granular scattering is developed, tested and presented.

4. Sensitivity of the extraction method to system noise is characterized.

5. Dependence of the extracted signature to the angle of incidence of the wave is determined.

\subsection{Summary of Dissertation}

Chapter 2 will introduce a terahertz measurement system and review the structure, types and composition of common explosives. This chapter will then review the theory behind the electromagnetic scattering of $\mathrm{THz}$ waves incident on material. The chapter continues with a review of rough surface statistics and current 
modeling methodologies and techniques for scattering caused by rough surfaces and granular media. At the end of the chapter, a simulation technique will be selected for the modeling work in this dissertation.

Chapter 3 will focus on the theory behind the Finite-Difference Time-Domain (FDTD) simulation method. In this chapter the basic time-stepping algorithm will be reviewed, as well as the use of absorbing boundary conditions (ABCs) to suppress spurious reflections, the Total-Field/Scattered-Field (TFSF) methodology to introduce plane waves into the simulation space, Near-to-Far-Field (NTFF) transformation for scattering calculations, the augmentation of the basic FDTD time-stepping algorithm to include dispersive media and validation of the FDTD methodology for this work. As part of the validation of the FDTD methods ability to simulate dispersive medium, the complex dielectric constant of the common explosive C-4 will be entered into the FDTD grid and the complex dielectric constant will be extracted and compared to measured results for consistency.

Chapter 4 will present measurements of granular media and rough surfaces at $\mathrm{THz}$ frequencies with comparisons to FDTD models as further validation of the FDTD model.

Chapter 5 will show how the random media interface masks the extracted spectral signature. This chapter will also discuss how to extract the spectral 
signature from the data and how sensitive the signature is to the angle of incidence and what signal-to-noise ratio (SNR) would be required for an actual measurement system.

Chapter 6 will detail the investigation of the granular scattering effect on the extracted spectral signature. The effect of noise in the measurement system will also be discussed.

Chapter 7 will combine the rough surface interface with the granular scattering to detail the most realistic case. The effect of noise in the measurement system will also be investigated.

Chapter 8 will summarize the findings of this work and present areas for additional research. 


\section{Chapter 2}

\section{Electromagnetic Scattering Theory}

Terahertz time-domain spectroscopy (THz-TDS) is gaining use for noninvasive scanning with applications including medical imaging, process monitoring and illicit material detection $[1,3,5]$. The basis of using THz-TDS for all of these applications is the theory of reflected and transmitted waves. In this chapter a brief review of explosive types and composition is given. Then the Picometrix TRay 4000 THz-TDS system at Portland State University is described. Next, the basic theory behind the reflected and transmitted waves on smooth and rough surfaces is reviewed. Finally, this chapter will also discuss various methods of modeling the reflected and transmitted waves incident against a rough surface and/or media containing granular scatterers.

\subsection{Components of Explosives}

Explosives are divided into three types (chemical, mechanical and nuclear) and

into two groups (high and low). Chemical explosives release energy in a chemical 
reaction. Common chemical explosives are organic materials such as compounds than contain nitrate groups. Mechanical explosives release energy mechanically, such as a compressed air cylinder. Nuclear explosives use the energy stored in atomic nuclei.

Low explosives have a detonation event that spreads through the material at subsonic speeds by an accelerated combustion process. This group of low explosives would be used to move objects such as rocks and rubble.

High explosives, which have a high detonation velocity, detonate supersonically and are driven by the breakdown of the molecular structure [19]. Because of the higher velocity, high explosive materials have much higher shattering power than low explosives and would be used for the demolition of objects.

A list of common explosive dielectric constants is given in Table 2.1 and a list of their make-up is given in Table 2.2 [22].

THz spectroscopy is useful for explosive detection because of the unique spectral fingerprint of individual explosive compounds, as seen in the spectral signature data of Table 2.3 .

The unique absorption spectra listed in Table 2.3 allow for the identification of the different types of explosives. However, the data shown in Table 2.3 are from pure samples which are perfectly prepared. As such, there is no rough surface scattering, and minimal granular scattering influencing the spectral peaks. Explosives typically found in practice are a mixture of the explosive material 
Table 2.1: Dielectric Constants of Explosive Grains.

\begin{tabular}{|c||c|l|c|}
\hline \multicolumn{4}{|c|}{ Dielectric of Explosive Grains } \\
\hline \hline Material & Direction & $\begin{array}{l}\text { Dielectric } \\
\text { Con- } \\
\text { stant }\end{array}$ & Reference \\
\hline PETN & 110 & $3.5 \pm 0.12$ & {$[23]$} \\
\hline & 001 & $4.75 \pm 0.17$ & {$[23]$} \\
\hline & 001 & $4.29 \pm 0.04$ & {$[23]$} \\
\hline RDX & 120 & $4.7 \pm 0.02$ & {$[23]$} \\
\hline & 110 & $4.25 \pm 0.11$ & {$[23]$} \\
\hline Tetryl & 011 & $4.88 \pm 0.16$ & {$[23]$} \\
\hline & 001 & $3.19 \pm 0.07$ & {$[23]$} \\
\hline TNT & 001 & $3.89 \pm 0.23$ & {$[23]$} \\
\hline HMX (1) & & 2.5249 & {$[24]$} \\
\hline & & 2.5408 & {$[24]$} \\
\hline & & 2.9929 & {$[24]$} \\
\hline
\end{tabular}

embedded in a plastic filler (5-20\% by volume including air voids). The mixture is typically comprised of air voids in the mixture a few percent by volume (see Figure 1.2 for a picture of an explosive mixture).

\section{$2.2 \quad$ Terahertz Measurement System}

The $\mathrm{THz}$ measurement system, the T-Ray 4000, developed by Picometrix is shown in Figure 2.1. The T-Ray 4000 has interchangeable fiber-coupled transmit and receive heads and all of the spectral software needed to perform either reflection or transmission mode measurements. The system at Portland State 
Table 2.2: Common mixtures of explosives in percentages from Meyer [22].

\begin{tabular}{|c|c|c|c|c|c|c|}
\hline \multicolumn{7}{|c|}{ Common Mixtures of Explosives } \\
\hline Explosive & PETN & RDX & TNT & $\mathrm{HMX}$ & $\begin{array}{c}\text { Addi- } \\
\text { tive }\end{array}$ & $\begin{array}{l}\text { Density } \\
(\mathrm{g} / \mathrm{cm} 3)\end{array}$ \\
\hline LX-04 & & & & $85 \%$ & $\begin{array}{c}15 \% \text { Viton } \\
\mathrm{A}\end{array}$ & 1.86 \\
\hline $\mathrm{C}$ & & $88.30 \%$ & & & $\begin{array}{l}11.7 \% \text { non } \\
\text { explosive } \\
\text { plasticizer }\end{array}$ & \\
\hline $\mathrm{C}-2$ & & $80 \%$ & & & $\begin{array}{l}20 \% \text { non } \\
\text { explosive } \\
\text { plasticizer }\end{array}$ & \\
\hline C-3 & & $78 \%$ & & & $\begin{array}{c}22 \% \text { non } \\
\text { explosive } \\
\text { plasticizer }\end{array}$ & \\
\hline $\mathrm{C}-4$ & & $90 \%$ & & & $\begin{array}{c}10 \% \text { poly- } \\
\text { isobuty- } \\
\text { lene }\end{array}$ & \\
\hline Semtex-H & $49.8 \%$ & $50.2 \%$ & & & $\begin{array}{c}\text { Styrene- } \\
\text { butadiene } \\
\text { rubber }\end{array}$ & \\
\hline A-3 & & $91 \%$ & & & $9 \%$ wax & 1.71 \\
\hline $\mathrm{B}$ & & $60 \%$ & $40 \%$ & & $\begin{array}{l}\text { wax as } \\
\text { additive }\end{array}$ & 1.65 \\
\hline
\end{tabular}

University has a $3 \mathrm{THz}$ bandwidth, greater than $70 \mathrm{~dB}$ peak Signal-to-Noise Ratio (SNR), rapid scan range of 320 ps and a rapid scan rate of $100 \mathrm{~Hz}$. A plot of the pulse in time and frequency are presented in Figure 2.2. The T-Ray 4000 is used for most of the measured data presented in this dissertation. 


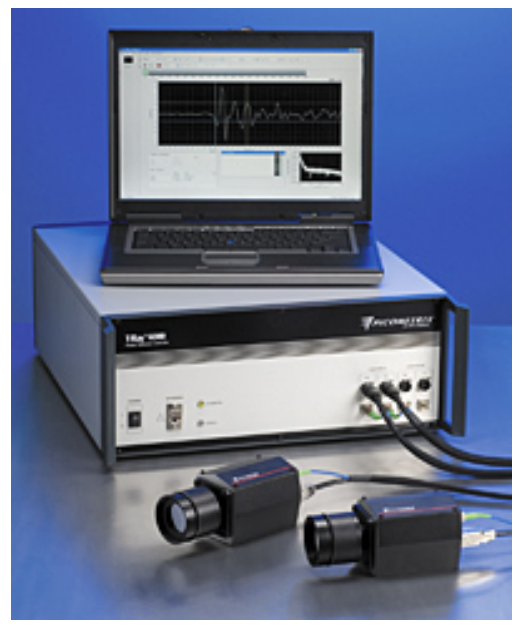

Figure 2.1: Photograph of T-Ray 4000 with transmit and receive heads.

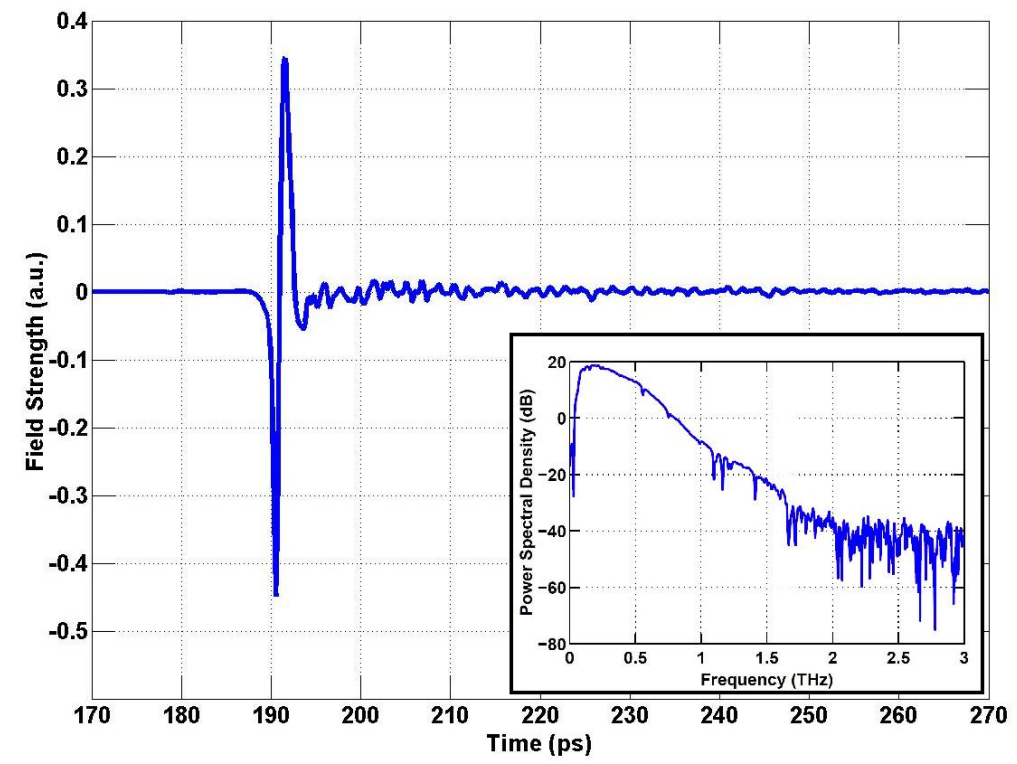

Figure 2.2: A THz pulse propagating in air measured with a Picometrix T-Ray 4000. Pulse duration is approximately 5 ps. Inset is the power spectral density (PSD) of the pulse. The usable portion of the spectrum spans from $0.1 \mathrm{THz}$ to $2 \mathrm{THz}$. 
Table 2.3: Location of Absorption Peaks for Explosive Crystals.

\begin{tabular}{|c|c|c|}
\hline \multicolumn{3}{|c|}{ Location of Spectral Peaks for Explosive Crystals } \\
\hline Explosive & THz center band & Reference \\
\hline $\begin{array}{c}\text { Semtex-H } \\
(\text { PETN and RDX) }\end{array}$ & $0.72,1.29,1.73,1.88,2.15,2.45,2.57$ & {$[11]$} \\
\hline RDX/C-4 & $0.72,1.26,1.73$ & {$[11],[25],[26]$} \\
\hline PETN & $1.73,2.51$ & {$[11]$} \\
\hline HMX & $1.58,1.91,2.21,2.57$ & {$[11]$} \\
\hline TNT & $1.44,1.91,5.6,8.2,9.1,9.9$ & {$[11],[26],[27]$} \\
\hline $\mathrm{NH}_{4} \mathrm{NO}_{3}$ & 4,7 & {$[28],[29]$} \\
\hline
\end{tabular}

\subsection{Fresnel Reflection and Transmission Coefficients}

The amount of energy reflected, or transmitted, at a flat dielectric interface is well understood [20, 30,31]. How much energy is reflected, or transmitted, depends upon the dielectric contrast, angle of incidence and the polarization of the electromagnetic wave. The amount of reflected and transmitted power is readily solved by applying the appropriate boundary condition at the dielectric interface [20].

\subsubsection{Normal Incidence}

A wave of normal incidence is show in Figure 2.3. It can be shown that the Fresnel reflection and transmission coefficients are [20]

$$
\Gamma=\frac{\eta_{2}-\eta_{1}}{\eta_{2}+\eta_{1}}
$$




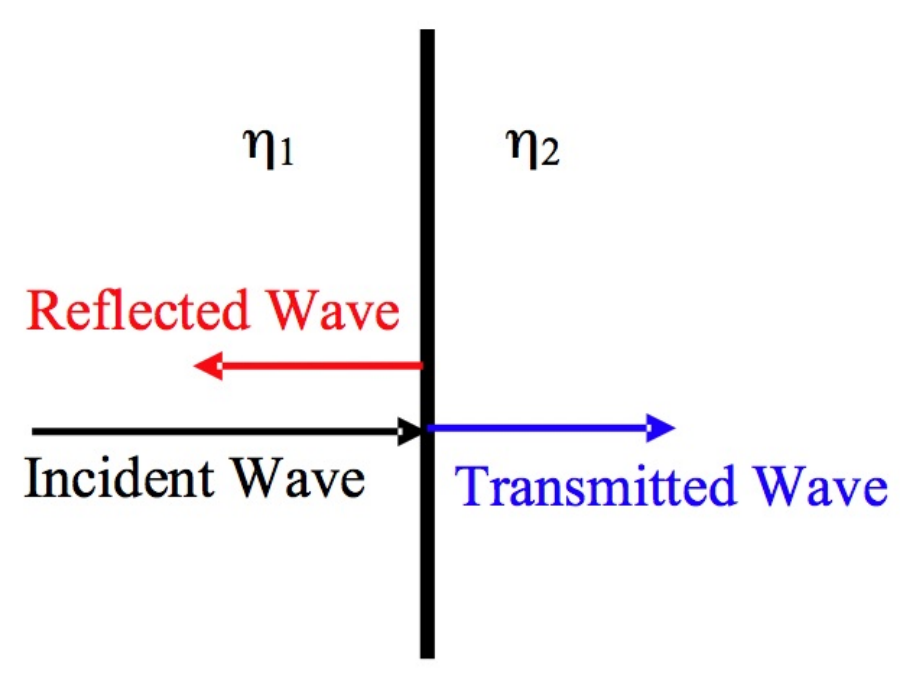

Figure 2.3: Normal incidence for either parallel or perpendicular polarization.

$$
T=1+\Gamma=\frac{2 \eta_{2}}{\eta_{2}+\eta_{1}}
$$

where $\Gamma$ is the reflection coefficient, $\mathrm{T}$ is the transmission coefficient, $\eta_{1}$ is the intrinsic impedance of the first medium and $\eta_{2}$ is the intrinsic impedance of the second medium. For all media $\eta$ is defined as [20]

$$
\eta=\sqrt{\frac{\mu}{\epsilon}}
$$

where $\mu$ is the permeability of the medium and $\epsilon$ is the permittivity of the medium. For lossless material, $\mu$ and $\epsilon$ will be purely real. For lossy or dispersive material, $\mu$ and $\epsilon$ will be complex [20]. 


\subsubsection{Oblique Incidence}

For waves incident on a dielectric half-space at an oblique angle (i.e., other than normal incidence), the Fresnel reflection and transmission coefficients are dependent on the incident angle as measured from the normal of the surface. An example of a plane wave at oblique incidence is shown in Figure 2.4. For parallel (vertical or $\mathrm{H}$ ) polarization the Fresnel reflection and transmission coefficients become $[20]$

$$
\begin{gathered}
\Gamma_{\|}=\frac{\eta_{2} \cos \left(\theta_{t}\right)-\eta_{1} \cos \left(\theta_{i}\right)}{\eta_{2} \cos \left(\theta_{t}\right)+\eta_{1} \cos \left(\theta_{i}\right)} \\
T_{\|}=1+\Gamma_{\|}=\frac{2 \eta_{2} \cos \left(\theta_{i}\right)}{\eta_{2} \cos \left(\theta_{t}\right)+\eta_{1} \cos \left(\theta_{i}\right)}
\end{gathered}
$$

which can also be written as

$$
\begin{gathered}
\Gamma_{\|}=\frac{\epsilon_{2} \beta_{1}-\epsilon_{1} \beta_{2} \sin \left(\theta_{t}\right)}{\epsilon_{2} \beta_{1}+\epsilon_{1} \beta_{2} \sin \left(\theta_{t}\right)} \\
T_{\|}=1+\Gamma_{\|}=\frac{2 \epsilon_{2} \beta_{1}}{\epsilon_{2} \beta_{1}+\epsilon_{1} \beta_{2} \sin \left(\theta_{t}\right)}
\end{gathered}
$$

and for perpendicular (horizontal or E) polarization the Fresnel reflection and transmission coefficients become [20]

$$
\begin{gathered}
\Gamma_{\perp}=\frac{\eta_{2} \cos \left(\theta_{i}\right)-\eta_{1} \cos \left(\theta_{t}\right)}{\eta_{2} \cos \left(\theta_{i}\right)+\eta_{1} \cos \left(\theta_{t}\right)} \\
T_{\perp}=1+\Gamma_{\perp}=\frac{2 \eta_{2} \cos \left(\theta_{i}\right)}{\eta_{2} \cos \left(\theta_{i}\right)+\eta_{1} \cos \left(\theta_{t}\right)}
\end{gathered}
$$




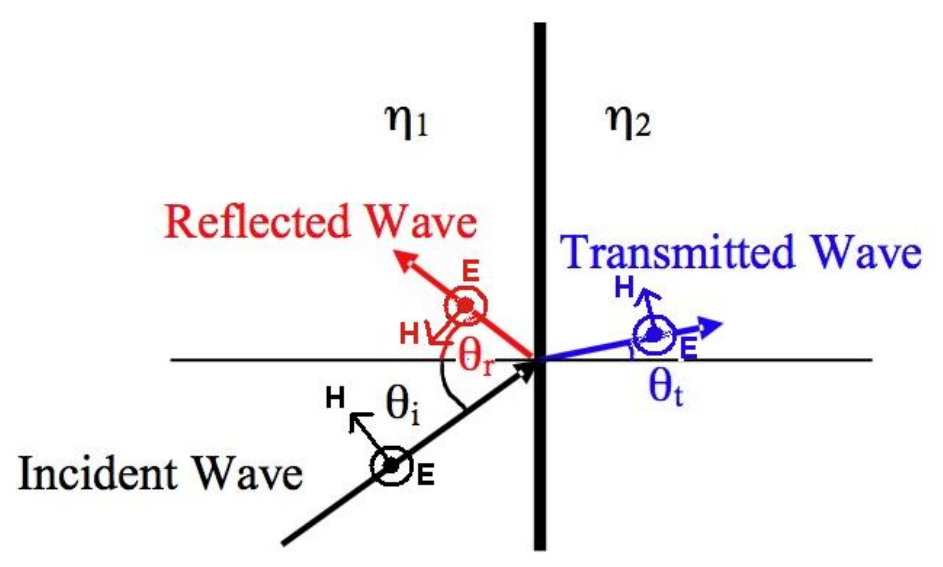

Figure 2.4: Oblique incidence for either parallel (E-field polarization parallel to the plane) or perpendicular polarization (E-field polarization perpendicular to the plane). Perpendicular polarization is illustrated in this figure.

which can also be written as

$$
\begin{gathered}
\Gamma_{\perp}=\frac{\mu_{2} \beta_{1}-\mu_{1} \beta_{2} \sin \left(\theta_{t}\right)}{\mu_{2} \beta_{1}+\mu_{1} \beta_{2} \sin \left(\theta_{t}\right)} \\
T_{\perp}=1+\Gamma_{\perp}=\frac{2 \mu_{2} \beta_{1}}{\mu_{2} \beta_{1}+\mu_{1} \beta_{2} \sin \left(\theta_{t}\right)}
\end{gathered}
$$

where the angle of reflection is given by the law of reflection, the angle of transmission is given by Snell's law of refraction.

$$
\begin{gathered}
\theta_{r}=\theta_{i} \quad \text { Law of Reflection } \\
\beta_{1} \sin \left(\theta_{i}\right)=\beta_{2} \sin \left(\theta_{t}\right) \quad \text { Snell's Law of Refraction }
\end{gathered}
$$

For Snell's Law of Refraction (Equation 2.13) $\beta$ is the wave number (often 


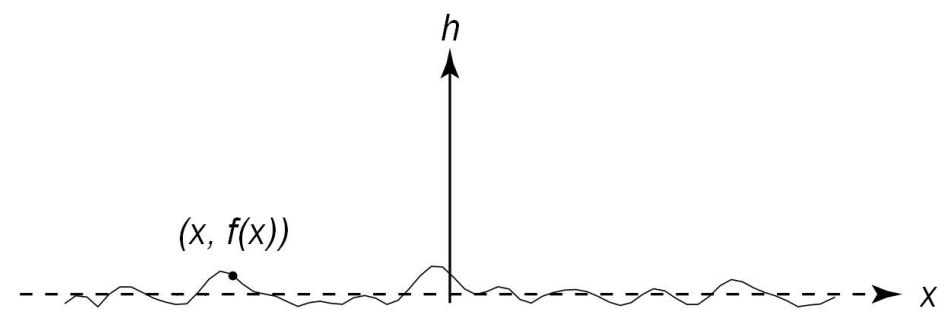

Figure 2.5: One-dimensional random rough surface

represented as $k$ ) for the media defined as $\beta=\omega \sqrt{\mu \epsilon}$ where $\omega$ is the radian frequency ( $2 \pi$ times frequency).

\subsection{Rough Surface Scattering}

As rough surface scattering is expected to have a large impact on the reflected and transmitted $\mathrm{THz}$ waves used for the detection of explosives, a review of rough surfaces and rough surface scattering is given here. To characterize the rough surface, the surface elevation with respect to some mean surface is assumed to be a stochastic process. The characterization of a random surface generally requires a multi-variate probability density function of surface heights. While most naturally occurring surfaces are not Gaussian, it is a reasonable approximation since a sufficient number of real surfaces will approach a Gaussian distribution. It is also usual for the surface to exhibit the same height statistics regardless of the spatial coordinates (i.e., it is stationary) [32]. 


\subsubsection{One-dimensional Gaussian Random Rough Surface}

The one-dimensional rough surface shown in Figure 2.5 has a height profile described by $f(x)$, which is a random function of the coordinate $x$. The surface height $z$ is described by the function $h=f(x)$. The function $f(x)$ has a Gaussian probability density function $p(h)$ as [32]

$$
p(h)=\frac{1}{\sigma_{s}^{2} \sqrt{2 \pi}} \exp \left(-\frac{(h-\tau)^{2}}{2 \sigma_{s}^{2}}\right)
$$

where $\sigma_{s}$ is the standard deviation or root-mean-square ( $\left.r m s\right)$ height and $\tau$ is the mean value of the surface height. The mean value of the surface height is typically assumed to be zero, which gives a surface centered about $h=0$.

The joint probability density function $p_{z 1 z 2}\left(z_{1}, z_{2}\right)$ of two Gaussian random variables is given by [32]

$$
p_{z 1 z 2}\left(z_{1}, z_{2}\right)=\frac{1}{2 \pi \sigma_{s 1} \sigma_{s 2} \sqrt{1-C^{2}}} \exp \left(-\frac{\frac{\left(z_{1}-\tau_{1}\right)^{2}}{\sigma_{s 1}^{2}}-\frac{2 C\left(z_{1}-\tau_{1}\right)\left(z_{2}-\tau_{2}\right)}{\sigma_{s 1} \sigma_{s 2}}+\frac{\left(z_{2}-\tau_{2}\right)^{2}}{\sigma_{s 2}^{2}}}{2\left(1-C^{2}\right)}\right)
$$

where $\tau_{1}$ and $\tau_{2}$ are the respective mean values of $z_{1}$ and $z_{2}$, and $\sigma_{s 1}^{2}$ and $\sigma_{s 2}^{2}$ are the variances. In equation (2.15), $\mathrm{C}$ is the correlation coefficient. If $\tau_{1}=\tau_{2}=0$, and $\sigma_{s 1}=\sigma_{s 2}=\sigma_{s}$, it can be shown that the covariance of the two random variables $z_{1}$ and $z_{2}$ is [32]

$$
<z_{1} z_{2}>=\sigma_{s}^{2} C
$$


where the correlation coefficient, $\mathrm{C}$, of two random variables $z_{1}$ and $z_{2}$ is generally defined as the ratio between their covariances and the product of their standard deviations $\sigma_{s 1} \sigma_{s 2}$ [32]. Note that $|C| \leq 1$. Also, if $\mathrm{C}=0$ then $z_{1}$ and $z_{2}$ are independent random variables.

For multiple variables, the correlation function of the random process of surface height $z=f(x)$ is defined as [32]

$$
R_{f}\left(x_{1}, x_{2}\right)=<f\left(x_{1}\right) f\left(x_{2}\right)>=\sigma_{s}^{2} C\left(x_{1}, x_{2}\right)
$$

where $C\left(x_{1}, x_{2}\right)$ is a function of $x_{1}$ and $x_{2}$. If the correlation function $C\left(x_{1}, x_{2}\right)$ is assumed to be Gaussian, then the correlation function $C\left(x_{1}, x_{2}\right)$ is expressed as $[32]$

$$
C\left(x_{1}, x_{2}\right)=\exp \left(\frac{\left(x_{1}-x_{2}\right)^{2}}{\ell_{C}}\right)
$$

where $\ell_{C}$ is the correlation length. As the distance between points $x_{1}$ and $x_{2}$ becomes much larger than $l$ (i.e., $\left.\left|x_{1}-x_{2}\right| \gg l\right) C\left(x_{1}, x_{2}\right)$ tends toward zero and the functions $f\left(x_{1}\right)$ and $f\left(x_{2}\right)$ become independent (i.e., un-correlated) [32].

\subsubsection{Rough Surface Scattering Fundamentals}

For the infinitely wide smooth surface (a sample of which is shown in Figure 2.6) the scattered energy is all reflected in the specular direction. That is, for a plane wave incident at $30^{\circ}$ from the normal, all of the reflected energy will be at $30^{\circ}$ 


\section{Air}

\section{Metal}

Figure 2.6: A sample of an infinitely wide smooth surface metal interface.

from the normal as shown in Figure 2.7. The response vs. angle is a delta function, with no energy making it into the other angles.

As the surface is made rough, more energy is scattered into the other angles. For a rough surface with a small rms height (i.e., not very rough) a small amount of energy is scattered into the other angles, but a large portion of the energy is still scattered in the specular direction. Figure 2.8 shows a rough surface with a small rms height (rms height $=0.05 \lambda$ and correlation length $0.2 \lambda$ where $\lambda$ is the free space wavelength) and Figure 2.9 shows the scattered energy vs. angle [32]. Notice that for this smoother surface, the specular peak is still visible in Figure 2.9. Since the surface in Figure 2.9 is defined in terms of the free space wavelength, the results are valid for any surface and frequency combination that 


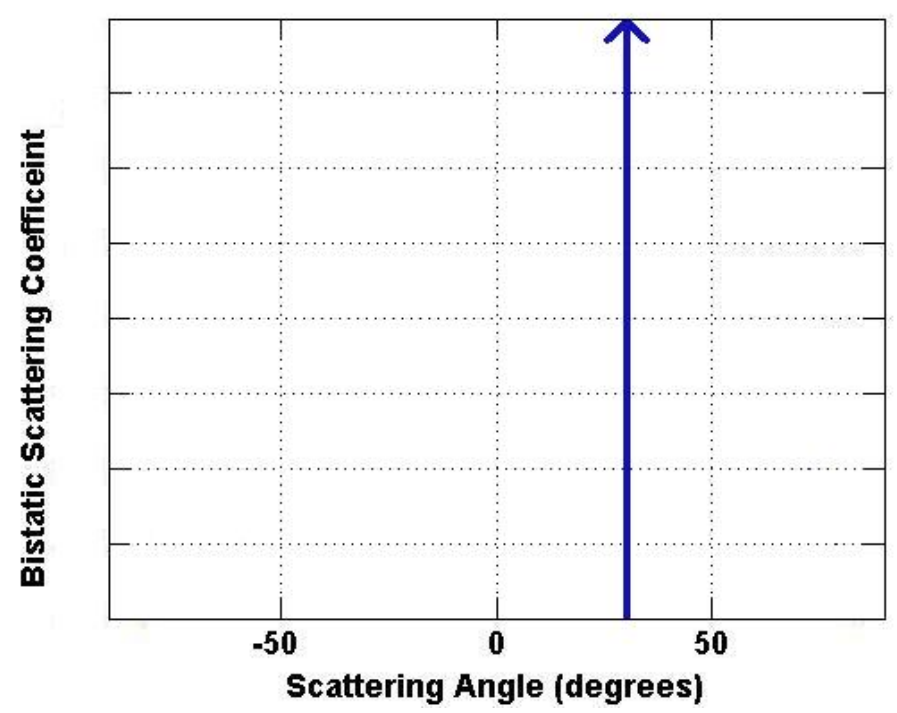

Figure 2.7: Smooth surface scattering for an incident wave at $30^{\circ}$ from the normal. All of the energy is reflected at $30^{\circ}$.

have an rms heigth of $0.05 \lambda$ and a correlation length of $0.2 \lambda$.

As the rough surface is made rougher (i.e., larger rms height) more energy is spread over the observation angles, with the limit being approximately equal energy at all angles for a sufficiently rough surface. Figure 2.10 shows a rough surface with a larger $\mathrm{rms}$ height ( $\mathrm{rms}$ height $=0.2 \lambda$ and correlation length $1.5 \lambda$ where $\lambda$ is the free space wavelength) with Figure 2.11 showing a large portion of the energy spread across much of the observation angle [32]. In this figure, the specular peak is lost because the incoherent scattering has a larger magnitude than the coherent scattering. Again, the results in Figure 2.11 are for a surface defined with the free space wavelength and the results are valid for any surface and frequency combination that have an rms heigth of $0.5 \lambda$ and a correlation 


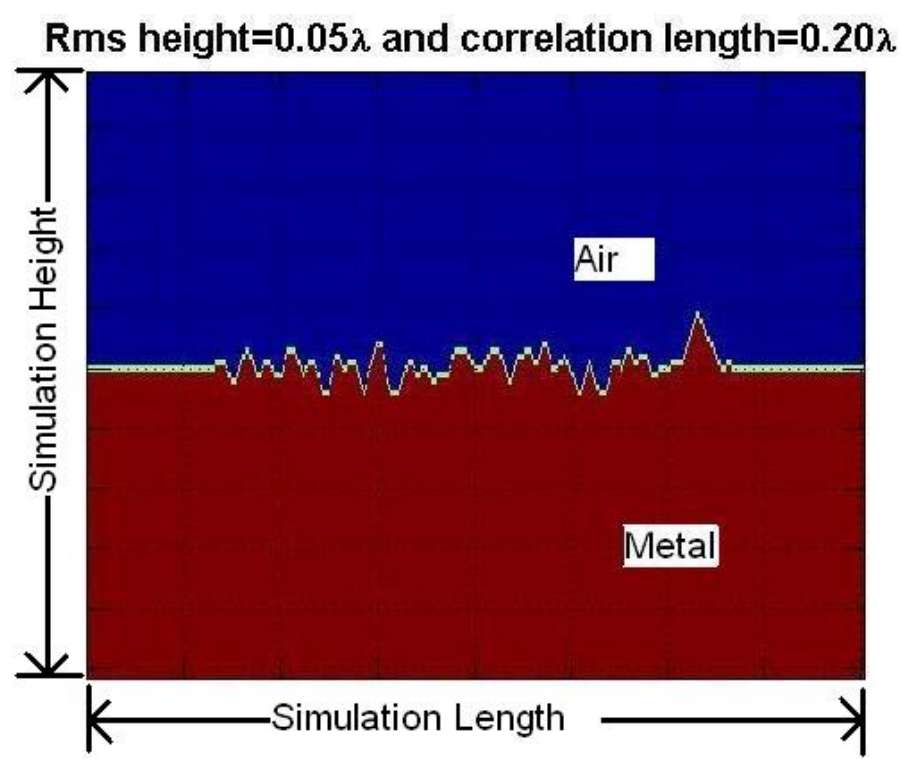

Figure 2.8: A rough surface metal interface with $\mathrm{h}_{r m s}=0.05 \lambda$ and $\mathrm{L}_{C}=0.2 \lambda(\lambda$ for free space).

length of $1.5 \lambda[32]$.

\subsubsection{Calculating Rough Surface Scattering}

There are several ways to calculate the scattering (or reflection) from a rough surface. The Fresnel Reflection and Transmission Coefficients discussed in the last section are for a smooth surface. However, the methodology used to calculate the Fresnel Reflection and Transmission Coefficients (i.e., applying the appropriate boundary conditions) is useful for determining the scattering from a rough surface. Some of the more widely used methods available to calculate rough surface scattering include:

1. Kirchhoff Approximation 


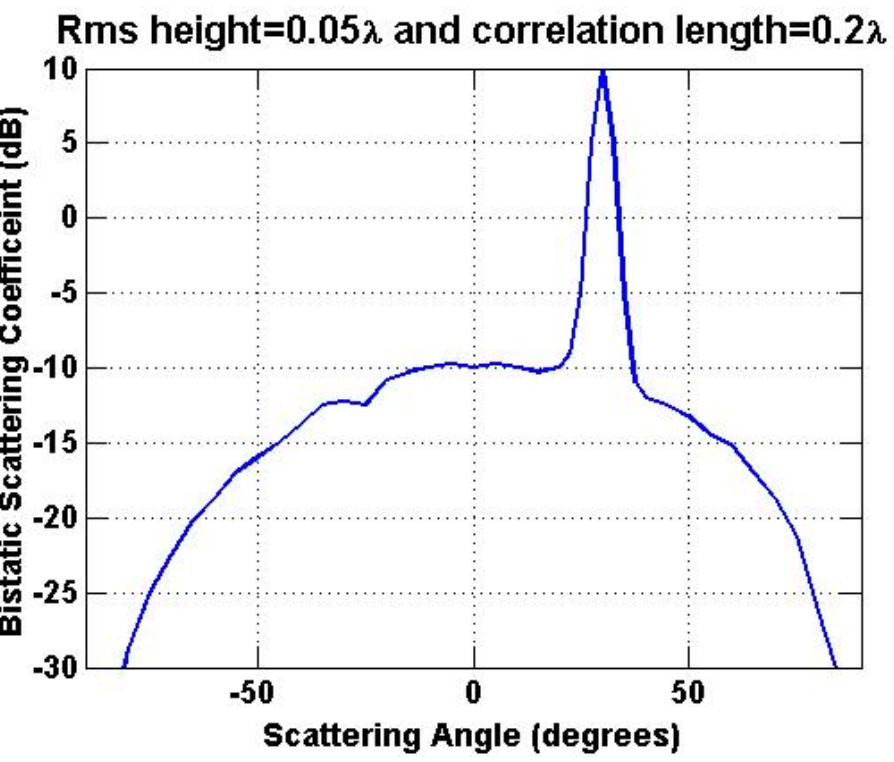

Figure 2.9: Rough surface scattering $\left(\mathrm{h}_{r m s}=0.05 \lambda\right.$ and $\mathrm{L}_{C}=0.2 \lambda, \lambda$ for free space) for an incident wave at $30^{\circ}$ from the normal. Most of the energy is reflected at $30^{\circ}$.

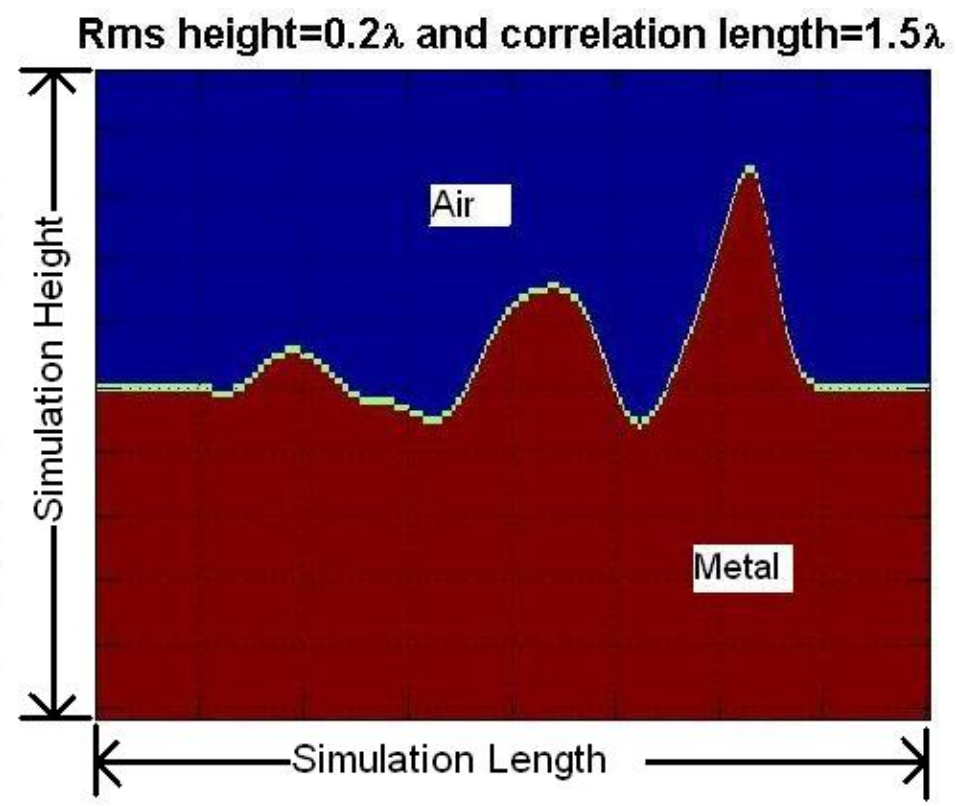

Figure 2.10: A rough surface metal interfacewith $\mathrm{h}_{r m s}=0.2 \lambda$ and $\mathrm{L}_{C}=1.5 \lambda(\lambda$ for free space). 


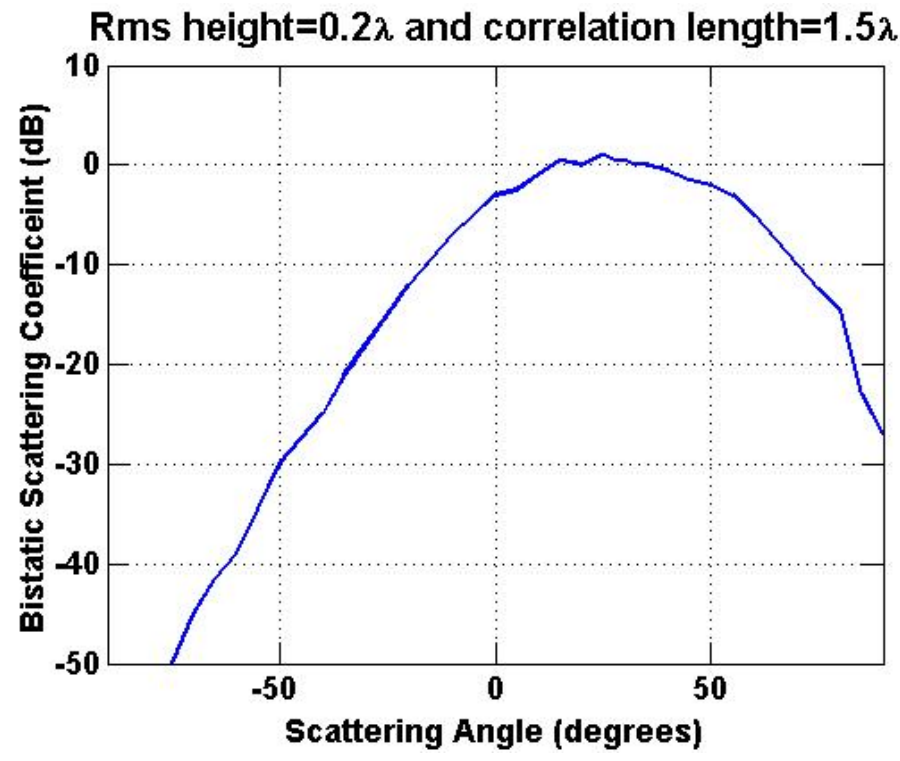

Figure 2.11: Rough surface scattering $\left(\mathrm{h}_{r m s}=0.2 \lambda\right.$ and $\mathrm{L}_{C}=1.5 \lambda, \lambda$ for free space) for an incident wave at $30^{\circ}$ from the normal. More energy is spread over a larger observation angle.

2. Small Perturbation Method (SPM)

3. Integral Equation Technique

4. Finite Element Method (FEM)

5. Finite-Difference Time-Domain (FDTD)

Analytical techniques often directly calculate the mean scattered field. $\mathrm{Nu}-$ merical techniques typically require a Monte-Carlo analysis to predict the rough surface scattering. For a Monte-Carlo analysis, a randomly rough surface is generated, and the scattering from that surface is calculated and saved. Then, a different randomly rough surface (using the same statistics as the prior surface) is generated and the scattering from that surface is also saved. This process of 
generating a new rough surface with the same statistics and calculating the scattering is repeated many times until the running average of the scattered electric field converges (i.e. less than $1 \%$ change from one pass to the next). Convergence is defined as when the running average stops changing, which is typically after 50-100 realizations of one dimensional surfaces for the surfaces explored in this work. The final result of a Monte-Carlo analysis is the average expected response for any surface with the simulated statistics.

\section{Kirchhoff Approximation}

The Kirchhoff Approximation applies the tangent plane approximation to allow for the calculation of the surface fields. The fields at any point on the rough surface are approximated by the fields that would be present on the tangent plane to that point. Because of this approximation, the Kirchhoff Approximation is valid only if the radius of curvature of the surface is larger than the wavelength (i.e., a slowly varying rough surface) [32].

\section{Small Perturbation Method}

The Small Perturbation Method (SPM) works by solving a similar, and simpler, problem. The solution to the simpler problem is then perturbed from slightly from the simple solution to more closely match the actual problem. An example of employing the SPM would be to calculate the orbit of the moon around 
the earth. The first solution would be to only take into account the gravity of the earth. Next, the solution could be perturbed to account for the gravity of the sun. If more accuracy was desired, the gravity from other planets in the solar system could be added as well. This assumption of a small change (i.e., a small perturbation) from the simpler problem limits the accuracy of the SPM to surfaces that are not very rough [33].

\section{Integral Equation Technique}

The Integral Equation (IE) Technique casts the solution for induced surface currents in the form of an integral equation where the unknown induced current density is part of the integrand [20]. Numerical methods, such as the Method of Moments (MoM), are then used to solve for the surface current density. Once the surface currents are known, the scattered fields can be calculated using traditional radiation integrals. Since no approximations are made about the current on the surface, the IE technique can be used for scattering from arbitrary surfaces.

\section{Finite Element Method}

The Finite Element Method (FEM) works by breaking up the simulation region into a mesh. The integral form of Maxwell's equations are then solved for the entire mesh in for each frequency point of interest. Since the mesh must be solved

for each frequency point of interest, generating broadband frequency data can be 
time consuming.

\section{Finite-Difference Time-Domain}

The Finite-Difference Time-Domain (FDTD) technique works by breaking up the region of interest into a grid. The point form of Maxwell's equations are then solved for the entire grid for a given point in time. Since the only approximation made with the FDTD method is the finite difference approximation, the FDTD method can be used for arbitrary surfaces and scattering objects [34]. Other benefits of the FDTD method include:

1. Arbitrary structures can be simulated.

2. Arbitrary materials can be simulated (including dispersive media).

3. Broadband frequency data obtained with one simulation pass.

\subsection{Granular Scattering}

Granular scattering is the scattering from small objects within material. Granular scattering is used with many applications including ground penetrating radar, medical, and security imaging and explosive detection. The granular scattering can be modeled with the following techniques:

1. Quasi-Crystalline Approximation

2. Born Approximation 
3. Rytov Approximation

4. Numerical Methods

\subsubsection{Quasi-Crystalline Approximation}

The Quasi-Crystalline Approximation (QCA) is an accurate method to calculate an effective dielectric constant for scattering problems. The QCA method will calculate a complex effective dielectric constant based on the size, density, and dielectric contrast of the granular scatterers in the background. The entire material is then modeled as a homogenous block of this complex valued dielectric material. QCA is based upon the multiple scattering that will occur between the granular particles embedded in the background (as shown in Figure 2.12). The multiple scattering within the sample is an infinite sum, but the QCA will truncate the sum assuming that statistical configurational averaging is used. The conditional probability is then truncated at the bivariate level to give an effective homogeneous complex dielectric constant for the material [32]. In fact the QCA method has been successfully used to granular scattering in the terahertz frequency band [35]. 


\subsubsection{Born Approximation}

The Born Approximation for granular scattering assumes there is negligible interaction between the multiple scatterers. This implies the internal field is approximated by the incident field which implies a small dielectric contrast. This approximation is commonly used for the reconstruction of medical images.

\subsubsection{Rytov Approximation}

A slightly more accurate approximation than the Born Approximation. Assumes there is limited interaction between the multiple scatterers. For the Born Approximation, the multiple scattering is ignored, but for the Rytov Approximation, the first interaction between the scatterers is included.

\subsubsection{Numerical Methods}

The prior three modeling methods make simplifying approximations to allow for the solution of the scattering events. Since numerical methods (i.e., the FDTD method) grid up the simulation space to solve Maxwell's equations, they do not make any approximations about the make-up or the number of reflection events to consider. Since the simulation space is discretized, approximations on the size and shape of the scatterers will be made which may effect the accuracy for curved surfaces. Error associated with the discrete nature of the simulation space can be minimized by making the size of the simulation cell (or mesh) 


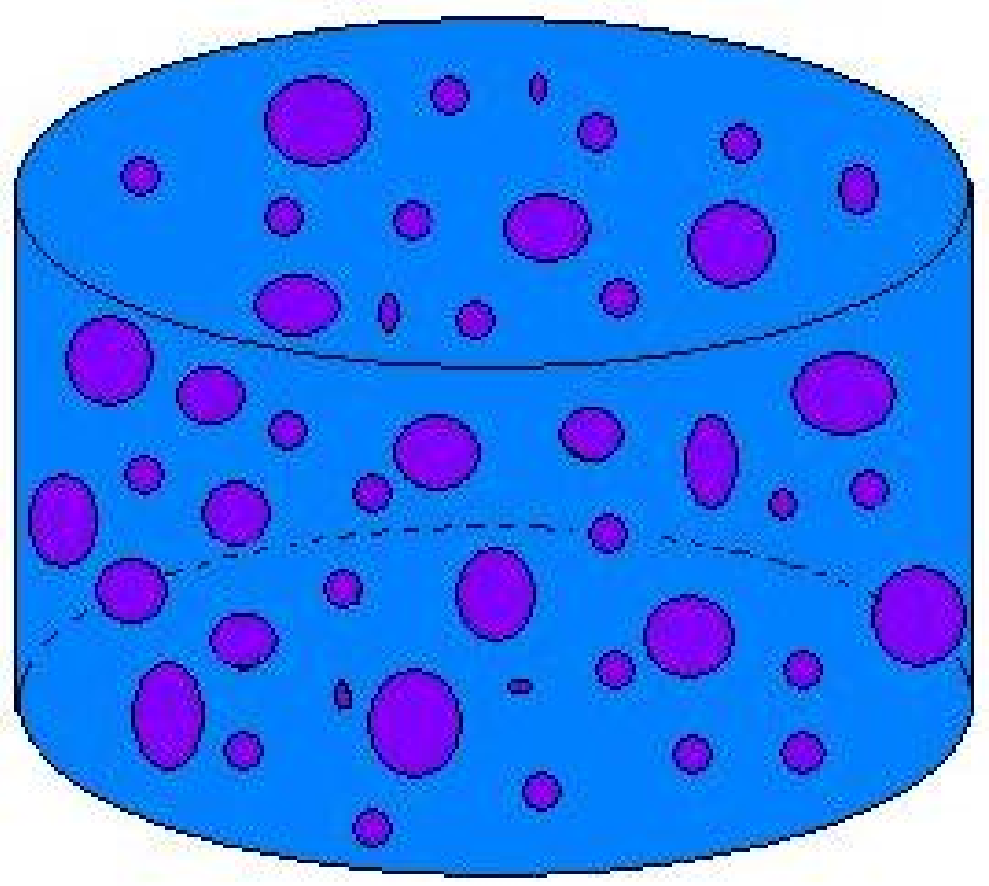

Figure 2.12: Sample of background material with granular scatterers.

small. Therefore, the numerical methods are generally applicable to all granular scattering problems.

\subsection{Selection of Modeling Technique}

There are many rough surface and granular scattering techniques outlined in the prior sections, and each technique is accurately applicable to a given situation. However, one technique is suited to the work necessary in this dissertation. The methodology selected for the work presented later in this dissertation is the FDTD technique. The FDTD technique was selected because of:

1. The ease of implementing inhomogeneous material (important for granular 
scatterers).

2. The ease of implementing dispersive material (important for the spectral signature of explosives).

3. The broadband frequency response gathered from a single FDTD simulation.

4. Validity for all rough surface scattering (unlike the Kirchhoff Approximation or SPM).

There are many benefits to selecting the FDTD simulation method, but there are some drawbacks which include:

1. Computationally intense (i.e., long simulation times).

2. More complex implementation than other modeling methods.

The first drawback may be addressed by process parallelization, or other methods, while the second drawback is a consequence of the versatility of the FDTD method.

\subsection{Conclusion}

An introduction to explosives was presented. Then the Picometrix T-Ray 4000 Terahertz measurement system was introduced. Next electromagnetic scattering was reviewed. The Fresnel reflection and transmission coefficients were presented, 
followed by a description of rough surface scattering. Various modeling methodologies for rough surface scattering were discussed. Next, granular scattering was reviewed. Finally, the FDTD method was selected as the modeling method of choice because of its versatility. 


\section{Chapter 3}

\section{The Finite-Difference Time-Domain Method}

The Finite-Difference Time-Domain (FDTD) method was originally introduced by Kane Yee in 1966 [21]. In this seminal paper, Yee chose a geometry for spatially sampling the electric and magnetic field vector components which represents both the differential and integral forms of Maxwell's Equations.

This chapter will outline the basic algorithm of the FDTD method as developed by Yee. This chapter will also outline the implementation of Absorbing Boundary Conditions (ABCs) to truncate the FDTD grid, plane wave sourcing by means of the Total-Field/Scattered-Field (TFSF) method, a Near-to-Far-Field (NTFF) transformation and the modification of the basic Yee algorithm to allow for the simulation of dispersive media. With all of these techniques defined for the FDTD method, a two-dimensional FDTD algorithm is programmed to model a one-dimensional rough surface. 


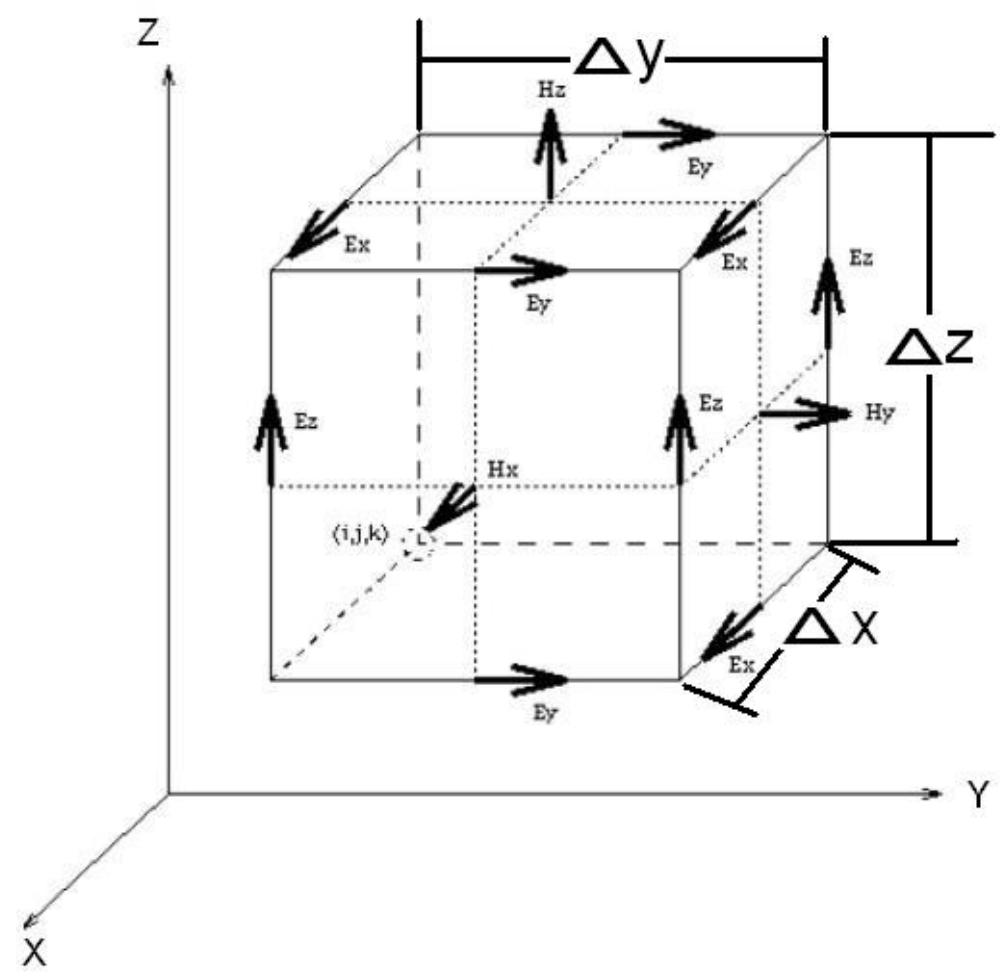

Figure 3.1: Position of the electric and magnetic field vector components about a cubic unit cell.

\subsection{Yee Algorithm}

The basic Yee Algorithm works by breaking up the space to be simulated into a grid of discrete points where the electric and magnetic field are sampled in space and time. This grid is shown in Figure 3.1. From this grid, the point form of Maxwell's equations can be expanded and represented by a finite central difference. 


\subsubsection{Maxwell's Equations}

Consider a region of space that may have materials that absorb or reflect electric and magnetic field energy. Then (using MKS units) Maxwell's Equations are given as $[20,30,31,34]$

$$
\begin{gathered}
\nabla \times \mathbf{E}=-\frac{\partial \mathbf{B}}{\partial t}-\mathbf{M} \quad \text { Faraday's Law } \\
\nabla \times \mathbf{H}=\frac{\partial \mathbf{D}}{\partial t}+\mathbf{J} \quad \text { Ampere's Law } \\
\nabla \cdot \mathbf{D}=\rho_{e} \quad \text { Gauss's Law for the electric field } \\
\nabla \cdot \mathbf{B}=\rho_{m} \quad \text { Gauss's Law for the magnetic field }
\end{gathered}
$$

where the parameters above are:

E : electric field (volts/meter)

D : electric flux density (coulombs / meter $^{2}$ )

H : magnetic field (amperes / meter)

B : magnetic flux density (webers / meter $^{2}$ ) 
$\mathbf{J}$ : electric current density (amperes / meter $^{2}$ )

M : equivalent magnetic current density (volts / meter $^{2}$ )

$\rho_{e}:$ electric charge density (coulombs $/$ meter $^{3}$ )

$\rho_{m}$ : equivalent magnetic charge density (webers $/$ meter $^{3}$ )

In linear, isotropic, nondispersive materials the vector quantities $\mathbf{D}$ and $\mathbf{B}$ can be related to $\mathbf{E}$ and $\mathbf{H}$, respectively by:

$$
\begin{gathered}
\mathbf{D}=\epsilon \mathbf{E}=\epsilon_{0} \epsilon_{r} \mathbf{E} \\
\mathbf{B}=\mu \mathbf{H}=\mu_{0} \mu_{r} \mathbf{H}
\end{gathered}
$$

where

$\epsilon:$ electrical permittivity (farads/meter)

$\epsilon_{0}$ : free-space permittivity $\left(8.854 \times 10^{-12}\right.$ farads/meter $)$

$\epsilon_{r}:$ relative permittivity (dimensionless scalar)

$\mu:$ magnetic permeability (henrys/meter)

$\mu_{0}:$ free-space permeability $\left(4 \pi \times 10^{-7}\right.$ henrys $/$ meter $)$

$\mu_{r}$ : relative permeability (dimensionless scalar) 
$\mathbf{J}$ and $\mathbf{M}$ can act as independent sources of $\mathbf{E}$ and $\mathbf{H}$ fields (call them $\mathbf{J}_{\text {source }}$ and $\left.\mathbf{M}_{\text {source }}\right)$. This will allow for the electric and magnetic current densities in equations (3.1) and (3.2) to written as:

$$
\begin{gathered}
\mathbf{J}=\mathbf{J}_{\text {source }}+\sigma \mathbf{E} \\
\mathbf{M}=\mathbf{M}_{\text {source }}+\sigma^{*} \mathbf{H}
\end{gathered}
$$

where $\sigma$ is the electrical conductivity (siemens/meter) and $\sigma^{*}$ is the equivalent magnetic loss (ohms/meter). This allows for materials that are isotropic and nondispersive to attenuate the electric and magnetic fields by conversion to heat.

Expanding the curl equations of Faraday's law and Ampere's law (equations 3.1 and 3.2 ) will yield:

$$
\begin{aligned}
& \frac{\partial H_{x}}{\partial t}=\frac{1}{\mu}\left[\frac{\partial E_{y}}{\partial z}-\frac{\partial E_{z}}{\partial y}-\left(M_{\text {source }_{x}}+\sigma^{*} H_{x}\right)\right] \\
& \frac{\partial H_{y}}{\partial t}=\frac{1}{\mu}\left[\frac{\partial E_{z}}{\partial x}-\frac{\partial E_{x}}{\partial z}-\left(M_{\text {source }_{y}}+\sigma^{*} H_{y}\right)\right] \\
& \frac{\partial H_{z}}{\partial t}=\frac{1}{\mu}\left[\frac{\partial E_{x}}{\partial y}-\frac{\partial E_{y}}{\partial x}-\left(M_{\text {source }_{z}}+\sigma^{*} H_{z}\right)\right] \\
& \frac{\partial E_{x}}{\partial t}=\frac{1}{\epsilon}\left[\frac{\partial H_{z}}{\partial y}-\frac{\partial H_{y}}{\partial z}-\left(J_{\text {source }_{x}}+\sigma E_{x}\right)\right] \\
& \frac{\partial E_{y}}{\partial t}=\frac{1}{\epsilon}\left[\frac{\partial H_{x}}{\partial z}-\frac{\partial H_{z}}{\partial x}-\left(J_{\text {source }_{y}}+\sigma E_{y}\right)\right]
\end{aligned}
$$




$$
\frac{\partial E_{z}}{\partial t}=\frac{1}{\epsilon}\left[\frac{\partial H_{y}}{\partial x}-\frac{\partial H_{x}}{\partial y}-\left(J_{\text {source }_{z}}+\sigma E_{z}\right)\right]
$$

The system of equations $(3.9$ - 3.14) forms the basis of the FDTD numerical algorithm for electromagnetic wave interactions with three dimensional objects.

While the original Yee algorithm was done for the lossless case (i.e., $\sigma$ and $\left.\sigma^{*}=0\right)[21]$, it still has many benefits since the fundamental idea was so robust. These benefits are:

1. The Yee algorithm solves for both electric and magnetic fields in time and space using the coupled Maxwell's equations. This allows for the modeling of radar cross section [34]. This also allows for the simulation of features unique to each field, such as tangential $\mathbf{H}$ singularities near edges and corners and radial $\mathbf{E}$ singularities near points and edges from the same simulation pass.

2. The finite-difference expressions for the space derivatives used for the curl operators are central-differences that are second-order accurate (accuracy increases as the square of the number of cells added to the simulation).

3. Continuity of $\mathbf{E}$ and $\mathbf{H}$ are maintained across an interface of dissimilar materials if the interface is parallel to one of the lattice coordinate axes.

4. The location of the $\mathbf{E}$ and $\mathbf{H}$ implicitly enforce Gauss' electric and magnetic law. 


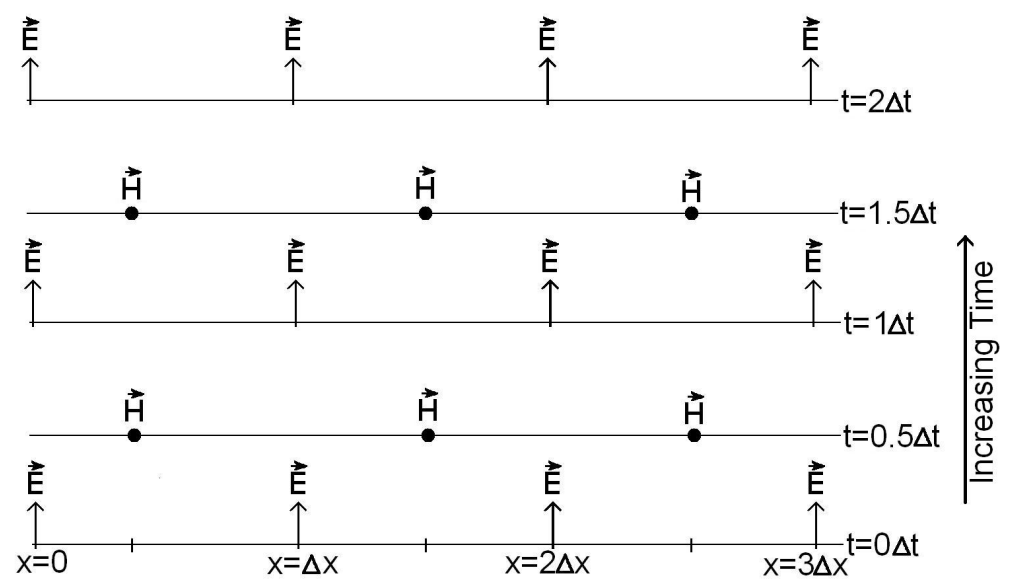

Figure 3.2: A one dimensional FDTD grid illustrating the leapfrog arrangement.

5. The Yee algorithm centers the $\mathbf{E}$ and $\mathbf{H}$ in time in what is termed a leapfrog arrangement. All of the $\mathbf{E}$ computations in the FDTD grid are completed and stored in memory. Then, the $\mathbf{H}$ computations are performed using the $\mathbf{E}$ from the prior time step. Next, the $\mathbf{E}$ data is re-computed for the FDTD grid using the $\mathbf{H}$ from the prior step. This procedure (illustrated in Figure 3.2) is continued until the time-stepping is concluded.

\subsubsection{Finite-Difference Notation}

The notation Yee introduced for the FDTD simulations for three dimensional space is $[21]$ :

$$
(i, j, k)=(i \Delta x, j \Delta y, k \Delta z)
$$

where $\Delta x, \Delta y$ and $\Delta x$ are the lattice space increments in $x, y$ and $z$ respectively with $i, j$, and $k$ being integers. A function of both space and time can be written 
in discrete form as:

$$
u(i \Delta x, j \Delta y, k \Delta z, n \Delta t)=u_{i, j, k}^{n}
$$

where $\Delta t$ is the time increment (assumed to be uniform over the simulation time) and $n$ is an integer.

Using centered finite-differencing, a partial derivative of the function $u$ in equation (3.16) with respect to a spatial coordinate gives:

$$
\frac{\partial u}{\partial x}(i \Delta x, j \Delta y, k \Delta z, n \Delta t)=\frac{u_{i+1 / 2, j, k}^{n}-u_{i-1 / 2, j, k}^{n}}{\Delta x}
$$

where the differencing is taken one half a grid cell to either side of the spatial index $i$ for the $x$ direction. A similar expression for a partial derivative of the function $u$ in equation (3.16) with respect to time is:

$$
\frac{\partial u}{\partial t}(i \Delta x, j \Delta y, k \Delta z, n \Delta t)=\frac{u_{i, j, k}^{n+1 / 2}-u_{i, j, k}^{n-1 / 2}}{\Delta t}
$$

\subsubsection{Maxwell's Equations in Finite-Difference Notation}

Using the notation introduced in equations (3.17) and (3.18) and plugging them into Maxwell's equations (using a semi-implicit scheme) as given in equations (3.9) 
- (3.14) gives (for a uniform, cubic grid with $\Delta x=\Delta y=\Delta z=\Delta$ ) [34]:

$$
\begin{aligned}
& \left.E_{x}\right|_{i, j+1 / 2, k+1 / 2} ^{n+1 / 2}= \\
& \left.\left.C_{a, E_{x}}\right|_{i, j+1 / 2, k+1 / 2} E_{x}\right|_{i, j+1 / 2, k+1 / 2} ^{n-1 / 2}+ \\
& \left.C_{b, E_{x}}\right|_{i, j+1 / 2, k+1 / 2} \cdot\left(\left.H_{z}\right|_{i, j+1, k+1 / 2} ^{n}-\left.H_{z}\right|_{i, j, k+1 / 2} ^{n}+\right. \\
& \left.\left.\quad H_{y}\right|_{i, j+1 / 2, k} ^{n}-\left.H_{y}\right|_{i, j+1 / 2, k+1} ^{n}-\left.J_{\text {source }}\right|_{i, j+1 / 2, k+1 / 2} ^{n} \Delta\right)
\end{aligned}
$$

$$
\begin{aligned}
& \left.E_{y}\right|_{i-1 / 2, j+1, k+1 / 2} ^{n+1 / 2}= \\
& \left.\left.\quad C_{a, E_{y}}\right|_{i-1 / 2, j+1, k+1 / 2} E_{y}\right|_{i-1 / 2, j+1, k+1 / 2} ^{n-1 / 2}+ \\
& \left.\quad C_{b, E_{y}}\right|_{i-1 / 2, j+1, k+1 / 2} \cdot\left(\left.H_{x}\right|_{i-1 / 2, j+1, k+1} ^{n}-\left.H_{x}\right|_{i-1 / 2, j+1, k} ^{n}+\right. \\
& \left.\left.\quad H_{z}\right|_{i-1, j+1, k+1 / 2} ^{n}-\left.H_{z}\right|_{i, j+1, k+1 / 2} ^{n}-\left.J_{\text {source }_{y}}\right|_{i-1 / 2, j+1, k+1 / 2} ^{n} \Delta\right)
\end{aligned}
$$

$$
\begin{aligned}
& \left.E_{z}\right|_{i-1 / 2, j+1 / 2, k+1} ^{n+1 / 2}= \\
& \left.\left.\quad C_{a, E_{z}}\right|_{i-1 / 2, j+1 / 2, k+1} E_{z}\right|_{i-1 / 2, j+1 / 2, k+1} ^{n-1 / 2}+ \\
& \left.\quad C_{b, E_{z}}\right|_{i-1 / 2, j+1 / 2, k+1} \cdot\left(\left.H_{y}\right|_{i, j+1 / 2, k+1} ^{n}-\left.H_{y}\right|_{i-1, j+1 / 2, k+1} ^{n}+\right. \\
& \left.\left.\quad H_{x}\right|_{i-1 / 2, j, k+1} ^{n}-\left.H_{x}\right|_{i-1 / 2, j+1, k+1} ^{n}-\left.J_{\text {source }}\right|_{i-1 / 2, j+1, k+1 / 2} ^{n} \Delta\right)
\end{aligned}
$$




$$
\begin{aligned}
& \left.H_{x}\right|_{i-1 / 2, j+1, k+1} ^{n+1}= \\
& \left.\left.\quad D_{a, H_{x}}\right|_{i-1 / 2, j+1, k+1} H_{x}\right|_{i-1 / 2, j+1, k+1} ^{n}+ \\
& \left.\quad D_{b, H_{x}}\right|_{i-1 / 2, j+1, k+1} \cdot\left(\left.E_{y}\right|_{i-1 / 2, j+1, k+3 / 2} ^{n+1 / 2}-\left.E_{y}\right|_{i-1 / 2, j+1, k+1 / 2} ^{n+1 / 2}+\right. \\
& \left.\left.\quad E_{z}\right|_{i-1 / 2, j+1 / 2, k+1} ^{n+1 / 2}-\left.E_{z}\right|_{i-1 / 2, j+3 / 2, k+1} ^{n+1 / 2}-\left.M_{\text {source }_{x}}\right|_{i-1 / 2, j+1, k+1} ^{n+1 / 2} \Delta\right)
\end{aligned}
$$

$$
\begin{aligned}
& \left.H_{y}\right|_{i, j+1 / 2, k+1} ^{n+1}= \\
& \left.\left.\quad D_{a, H_{y}}\right|_{i, j+1 / 2, k+1} H_{y}\right|_{i, j+1 / 2, k+1} ^{n}+ \\
& \left.\quad D_{b, H_{y}}\right|_{i, j+1 / 2, k+1} \cdot\left(\left.E_{z}\right|_{i+1 / 2, j+1 / 2, k+1} ^{n+1 / 2}-\left.E_{z}\right|_{i-1 / 2, j+1 / 2, k+1} ^{n+1 / 2}+\right. \\
& \left.\left.\quad E_{x}\right|_{i, j+1 / 2, k+1 / 2} ^{n+1 / 2}-\left.E_{x}\right|_{i, j+1 / 2, k+3 / 2} ^{n+1 / 2}-\left.M_{\text {source }_{y}}\right|_{i, j+1 / 2, k+1} ^{n+1 / 2} \Delta\right)
\end{aligned}
$$

$$
\begin{aligned}
& \left.H_{z}\right|_{i, j+1, k+1 / 2} ^{n+1}= \\
& \left.\left.\quad D_{a, H_{z}}\right|_{i, j+1, k+1 / 2} H_{z}\right|_{i, j+1, k+1 / 2} ^{n}+ \\
& \left.\quad D_{b, H_{z}}\right|_{i, j+1, k+1 / 2} \cdot\left(\left.E_{x}\right|_{i, j+3 / 2, k+1 / 2} ^{n+1 / 2}-\left.E_{x}\right|_{i, j+1 / 2, k+1 / 2} ^{n+1 / 2}+\right. \\
& \left.\left.\quad E_{y}\right|_{i-1 / 2, j+1, k+1 / 2} ^{n+1 / 2}-\left.E_{y}\right|_{i+1 / 2, j+1, k+1 / 2} ^{n+1 / 2}-\left.M_{\text {source }_{z}}\right|_{i, j+1, k+1 / 2} ^{n+1 / 2} \Delta\right)
\end{aligned}
$$

where the coefficients $C_{a}, C_{b}, D_{a}$ and $D_{b}$ are called updating coefficients. It is in these coefficients where the material parameters for each grid point (i.e., the 
permittivity, permeability and conductivity) are stored. In general, the updating coefficients may be a different value for each grid point.

$$
\begin{aligned}
&\left.C_{a}\right|_{i, j, k}=\frac{1-\frac{\sigma_{i, j, k} \Delta t}{2 \epsilon_{i, j, k}}}{1+\frac{\sigma_{i, j, k} \Delta t}{2 \epsilon_{i, j, k}}} \\
&\left.C_{b}\right|_{i, j, k}= \frac{\frac{\Delta t}{\epsilon_{i, j, k} \Delta}}{1+\frac{\sigma_{i, j, k} \Delta t}{2 \epsilon_{i, j, k}}} \\
&\left.D_{a}\right|_{i, j, k}= 1-\frac{\sigma_{i, j, k}^{*} \Delta t}{2 \mu_{i, j, k}} \\
& 1+\frac{\sigma_{i, j, k}^{*} \Delta t}{2 \mu_{i, j, k}} \\
&\left.D_{b}\right|_{i, j, k}=\frac{\frac{\Delta t}{\mu_{i, j, j} \Delta}}{1+\frac{\sigma_{i, j, k}^{*} \Delta t}{2 \mu_{i, j, k}}}
\end{aligned}
$$

The equations (3.19) - (3.24) listed, form the backbone for all FDTD simulations. These equations will update the $\mathbf{E}$ and $\mathbf{H}$ fields for the entire grid. Other issues that must be addressed, such as grid truncation and introducing sources into the FDTD grid, will be addressed in the following sections.

The selection of the grid size $(\Delta)$ and the time step size $(\Delta t)$ is dependent upon the Courant Stability. If the dispersion equation is solved for one-, two- or three-dimensions, the following Courant Stability number is obtained [34]:

$$
\begin{aligned}
& S_{1 D}=1 \\
& S_{2 D}=\frac{1}{\sqrt{2}} \\
& S_{3 D}=\frac{1}{\sqrt{3}}
\end{aligned}
$$


where the Courant Stability is equal to:

$$
S=\frac{c \Delta t}{\Delta x}
$$

Typically, the grid size $(\Delta x)$ is selected first to get at least 20 cells per wavelength at the highest frequency of interest. Then (to minimize dispersion in the FTDT grid [34]) the Courant Stability $(S)$ is selected to be at the one-, twoor three-dimensional limits given above. Finally, the optimum time-step for the FDTD simulation is computed using equation (3.29).

\subsection{FDTD Sources: The Total-Field/Scattered-Field Method}

The introduction of electromagnetic waves to a FDTD grid is necessary for the solution of many electromagnetic scattering problems. There are many methods for the introduction of an electromagnetic wave into the FDTD grid [34]. Three examples are:

1. Hard-sources of $\mathbf{E}$ and $\mathbf{H}$ fields in one- and two-dimensional grids.

2. $\mathbf{J}$ and $\mathbf{M}$ current sources for three dimensional grids.

3. The total-field/scattered-field (TFSF) formulation for plane-wave excitation in one-, two- and three-dimensional grids. 
As the work done in this dissertation required the introduction of a plane-wave into the FDTD grid, only the TFSF method will be discussed here.

The development of a plane-wave source introduction was discussed in the original paper by Yee [21]. In this paper Yee inserted the incident wave as an initial condition at each $\mathbf{E}$ and $\mathbf{H}$ component location in the FDTD grid. However, this original approach to plane-wave sourcing had two profound problems [34]:

1. The grid must be physically large to contain long-duration pulses or continuous sinusoids as sources. This wastes computer resources.

2. A wave sourced by this method will, at an oblique angle in a two- or threedimensional grid, undergo distortion of the wavefront as it drags against the outer grid boundary.

The TFSF method is the result of work to eliminate the problems listed above $[36,37,38]$. The TFSF method is based on the linearity of Maxwell's equations. It assumes that the physical total electric and magnetic fields $\left(\mathbf{E}_{\text {total }}\right.$ and $\left.\mathbf{H}_{\text {total }}\right)$ can be written as:

$$
\begin{aligned}
& \mathrm{E}_{\text {total }}=\mathrm{E}_{\mathrm{inc}}+\mathrm{E}_{\text {scat }} \\
& \mathrm{H}_{\text {total }}=\mathrm{H}_{\text {inc }}+\mathrm{H}_{\text {scat }}
\end{aligned}
$$


where $\mathbf{E}_{\mathbf{i n c}}$ and $\mathbf{H}_{\mathbf{i n c}}$ are the values of the incident-wave field (i.e., the source wave), which are assumed to be known at all points of the FDTD grid at all time-steps. The incident fields are the fields that would exist in free-space if there were no material (other than vacuum) in the FDTD grid. $\mathbf{E}_{\text {scat }}$ and $\mathbf{H}_{\text {scat }}$ are the values for the scattered-wave fields that result from any incident wave interactions with the material in the FDTD grid.

It is worth stating, that the Yee formulation for updating the $\mathbf{E}$ and $\mathbf{H}$ fields in the FDTD grid can be applied with equal validity to the incident field, the scattered field, and the total field. Figure 3.3 shows a two-dimensional FDTD grid broken into two regions. One region (Region 1) is the portion of the FDTD grid where both the incident and scattered fields co-exist (i.e., the total-field region). The other region (Region 2) is the portion of the FDTD grid where only the scattered field is allowed to exist (i.e., the scattered-field region). Region 1 and Region 2 are separated by a non-physical boundary that serves to connect the fields in the two regions, which is used to introduce the incident wave [34]. The edge of the simulation space (labeled "Lattice Truncation" in Figure 3.3) is where the FDTD grid is terminated. The lattice termination is discussed in the next section.

The TFSF formulation has many advantages that increase the computational flexibility and dynamic range of the FDTD method as listed below [34]. 


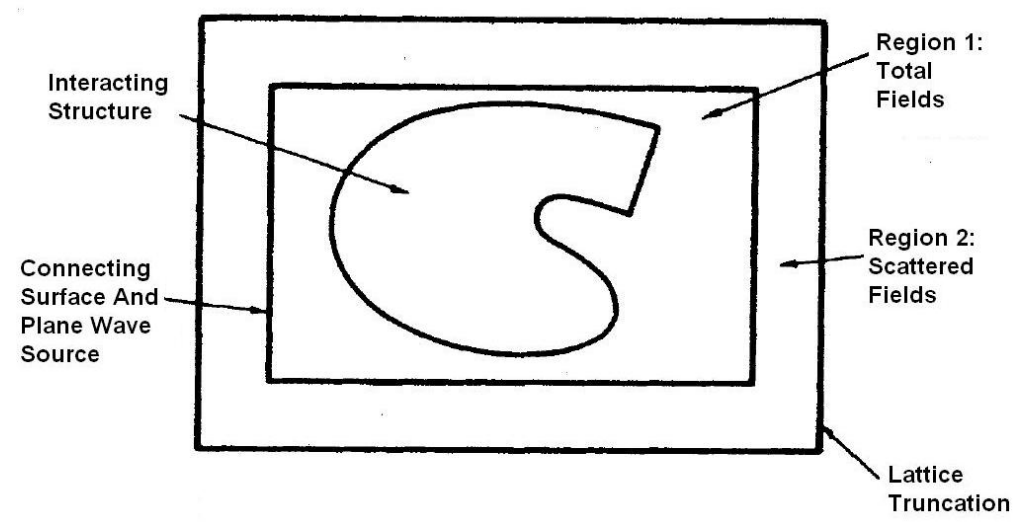

Figure 3.3: Showing a FDTD grid with a total field region and scattered field region [34].

1. Arbitrary incident wave angles.

2. Straightforward to modify the FDTD update equations to allow for the total-field and scattered-field regions.

3. Increased computational dynamic range of low levels of scattering.

4. Enhanced performance of absorbing boundary conditions (discussed in the next section) for lattice truncations since the lower level scattered fields are all that exist at the FDTD grid edges.

5. Far-Field scattering is easier to calculate from the scattered field region since the total $\mathrm{E}$ and $\mathrm{H}$ fields have already been removed from the fields in the FDTD grid.

It is easier to illustrate the TFSF method in two-dimensions. The $\mathrm{TM}_{z}$ formulation (i.e., perpendicular polarization) is arbitrarily chosen for the illustration of 
the TFSF methodology. For the $\mathrm{TM}_{z}$ polarization, the FDTD update equations reduce to (with no sources):

$$
\begin{aligned}
& \left.E_{z}\right|_{i-1 / 2, j+1 / 2, k+1} ^{n+1 / 2}= \\
& \left.\left.\quad C_{a, E_{x}}\right|_{i-1 / 2, j+1 / 2, k+1} E_{z}\right|_{i-1 / 2, j+1 / 2, k+1} ^{n-1 / 2}+\left.C_{b, E_{z}}\right|_{i-1 / 2, j+1 / 2, k+1} . \\
& \quad\left(\left.H_{y}\right|_{i, j+1 / 2, k+1} ^{n}-\left.H_{y}\right|_{i-1, j+1 / 2, k+1} ^{n}+\left.H_{x}\right|_{i-1 / 2, j, k+1} ^{n}-\left.H_{x}\right|_{i-1 / 2, j+1, k+1} ^{n}\right)(3.3
\end{aligned}
$$

$$
\begin{aligned}
& \left.H_{x}\right|_{i-1 / 2, j+1, k+1} ^{n+1}= \\
& \left.\left.\quad D_{a, H_{x}}\right|_{i-1 / 2, j+1, k+1} H_{x}\right|_{i-1 / 2, j+1, k+1} ^{n}+ \\
& \left.\quad D_{b, H_{x}}\right|_{i-1 / 2, j+1, k+1} \cdot\left(\left.E_{z}\right|_{i-1 / 2, j+1 / 2, k+1} ^{n+1 / 2}-\left.E_{z}\right|_{i-1 / 2, j+3 / 2, k+1} ^{n+1 / 2}\right)
\end{aligned}
$$

$$
\begin{aligned}
& \left.H_{y}\right|_{i, j+1 / 2, k+1} ^{n+1}= \\
& \left.\left.\quad D_{a, H_{y}}\right|_{i, j+1 / 2, k+1} H_{y}\right|_{i, j+1 / 2, k+1} ^{n}+ \\
& \left.\quad D_{b, H_{y}}\right|_{i, j+1 / 2, k+1} \cdot\left(\left.E_{z}\right|_{i+1 / 2, j+1 / 2, k+1} ^{n+1 / 2}-\left.E_{z}\right|_{i-1 / 2, j+1 / 2, k+1} ^{n+1 / 2}\right)
\end{aligned}
$$

Within a FDTD grid (as shown in Figure 3.3) the normal Yee algorithm can be applied to both the total-field and scattered-field regions without any problems. In fact, the Yee algorithm can be applied to the entire FDTD grid with a TFSF boundary without a loss of accuracy. The only issue that exists 


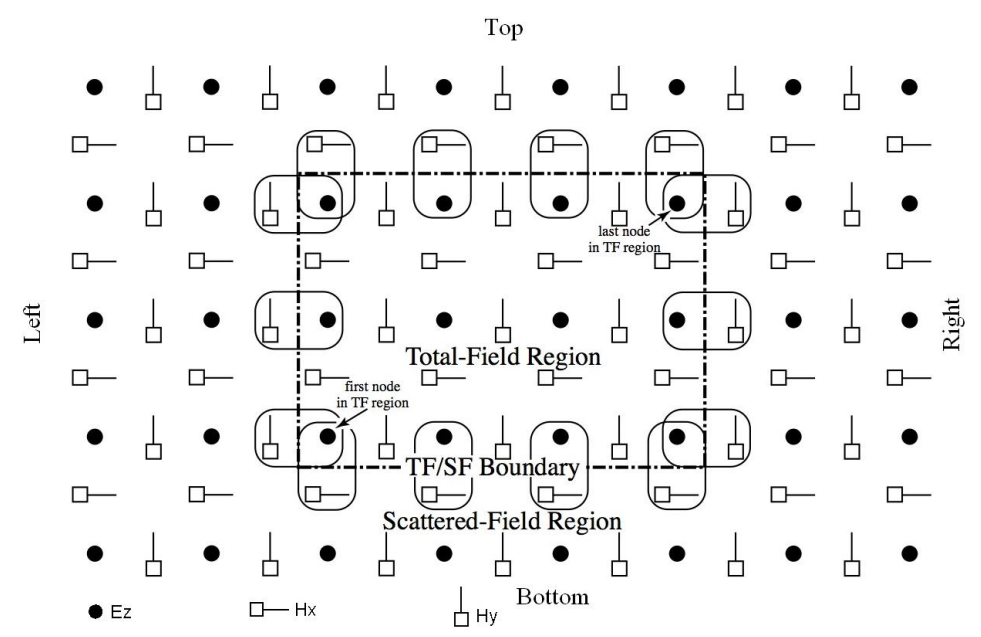

Figure 3.4: The FDTD grid showing each field point and the TFSF boundary [39].

is the updating at the TFSF boundary (labeled "Connecting Surface and Plane Wave Source" in Figure 3.3). For the $\mathbf{E}$ and $\mathbf{H}$ being updated right at the TFSF boundary, the fields to one side of the boundary are the total-field and the the fields to the other side of the boundary are the scattered-field. To resolve this discontinuity, at the TFSF boundary, the source wave (i.e., the incident wave) is either added or subtracted to the $\mathbf{E}$ and $\mathbf{H}$ fields to enforce the continuity of power at this point.

A FDTD grid showing each field grid point, and the TFSF boundary is shown in Figure 3.4 [39]. The circled $\mathrm{E}$ and $\mathrm{H}$ nodes are the nodes in the FDTD that must be corrected to ensure continuity across the TFSF boundary. The correcting equations for both the electric and magnetic field points are: 


$$
\begin{aligned}
& \left.E_{z}^{\prime}\right|_{i, j_{b o t}} ^{n+1}=\left.E_{z}\right|_{i, j_{b o t}} ^{n+1}+\left.\frac{\Delta t}{\epsilon_{0} \Delta} H_{x, i n c}\right|_{i, j_{b o t}-1 / 2} ^{n+1 / 2} \quad \text { Bottom TFSF boundary } \\
& \left.E_{z}^{\prime}\right|_{i, j_{\text {top }}} ^{n+1}=\left.E_{z}\right|_{i, j_{\text {top }}} ^{n+1}-\left.\frac{\Delta t}{\epsilon_{0} \Delta} H_{x, i n c}\right|_{i, j_{\text {top }}+1 / 2} ^{n+1 / 2} \quad \text { Top TFSF boundary } \\
& \left.E_{z}^{\prime}\right|_{i_{l e f t}, j} ^{n+1}=\left.E_{z}\right|_{i_{l e f t}, j} ^{n+1}-\left.\frac{\Delta t}{\epsilon_{0} \Delta} H_{y, i n c}\right|_{i_{l e f t}-1 / 2, j} ^{n+1 / 2} \quad \text { Left TFSF boundary } \\
& \left.E_{z}^{\prime}\right|_{i_{\text {right }}, j} ^{n+1}=\left.E_{z}\right|_{i_{\text {right }}, j} ^{n+1}+\left.\frac{\Delta t}{\epsilon_{0} \Delta} H_{y, i n c}\right|_{i_{\text {right }}+1 / 2, j} ^{n+1 / 2} \quad \text { Right TFSF boundary } \\
& \left.H_{x}^{\prime}\right|_{i, j_{b o t}-1 / 2} ^{n+1 / 2}=\left.H_{x}\right|_{i, j_{b o t}-1 / 2} ^{n+1 / 2}+\left.\frac{\Delta t}{\mu_{0} \Delta} E_{z, i n c}\right|_{i, j_{b o t}} ^{n} \quad \text { Bottom TFSF boundary } \\
& \left.H_{x}^{\prime}\right|_{i, j_{t o p}+1 / 2} ^{n+1 / 2}=\left.H_{x}\right|_{i, j_{t o p}+1 / 2} ^{n+1 / 2}-\left.\frac{\Delta t}{\mu_{0} \Delta} E_{z, \text { inc }}\right|_{i, j_{t o p}} ^{n} \quad \text { Top TFSF boundary } \\
& \left.H_{y}^{\prime}\right|_{i_{l e f t}-1 / 2, j} ^{n+1 / 2}=\left.H_{y}\right|_{i_{l e f t}-1 / 2, j} ^{n+1 / 2}-\left.\frac{\Delta t}{\mu_{0} \Delta} E_{z, i n c}\right|_{i_{t o p}, j} ^{n} \quad \text { Left TFSF boundary } \\
& \left.H_{y}^{\prime}\right|_{i_{\text {right }}+1 / 2, j} ^{n+1 / 2}=\left.H_{y}\right|_{i_{\text {right }}+1 / 2, j} ^{n+1 / 2}+\left.\frac{\Delta t}{\mu_{0} \Delta} E_{z, \text { inc }}\right|_{i_{\text {right }}, j} ^{n} \quad \text { Right TFSF boundary }
\end{aligned}
$$

where $\mathrm{E}_{z, \text { inc }}, \mathrm{H}_{x, \text { inc }}$ and $\mathrm{H}_{y, \text { inc }}$ are the assumed known incident plane wave with values known for all time. The primed field quantities are the $\mathrm{E}$ or $\mathrm{H}$ field values at the TFSF boundary after the TFSF correction is performed. This planewave can be calculated by using either an auxiliary one-dimensional grid [34] or by what is called the Analytic Field Propagation (AFP) method [39, 40]. The auxiliary grid works well for plane waves that have a direction of propagation that is parallel to one of the FDTD grid axes. The AFP method provides superior 
performance for plane-waves propagating at arbitrary angles to the FDTD grid axes. Also, the AFP method is more applicable to half-plane interfaces (i.e., semi-infinite air/dielectric interfaces). Because of the increased accuracy of the AFP method, as well as its applicability to planar interfaces, the AFP method was selected for the plane-wave sourcing for the work in this dissertation.

\subsubsection{Analytic Field Propagation Method}

In any FDTD grid, a wave will travel slower than a wave in the analog world. Moreover, the velocity at which a wave propagates in the FDTD grid changes with frequency (i.e., it is dispersive) [41]. Therefore, any time-domain pulse (which will have a significant amount of energy over a wide frequency range) will be spread out by the dispersion of the FDTD grid. This problem makes the implementation of a TFSF boundary for the sourcing of a plane-wave difficult. The Analytic Field Propagation (AFP) method address this issue to create a nearly perfect introduction of the plane-wave into the FDTD grid by [40]:

1. Solving the dispersion relation for the FDTD grid.

$$
\frac{1}{S^{2}} \sin ^{2}\left(\frac{\omega \Delta t}{2}\right)=\sin ^{2}\left(\frac{k_{x} \Delta}{2}\right)+\sin ^{2}\left(\frac{k_{y} \Delta}{2}\right)+\sin ^{2}\left(\frac{k_{z} \Delta}{2}\right)
$$

where the $k_{x, y, z}$ are the wave numbers for the $\mathrm{x}, \mathrm{y}$ and $\mathrm{z}$ directions. This relationship can be efficiently solved using the Newton method of root 
finding.

2. Selecting a reference node for the introduction of a plane-wave into the FDTD grid. This node is selected as the zero phase point for the propagating plane wave.

3. Calculate the wave number in the FDTD grid (using the solved dispersion relationship from step 1).

4. Calculate the magnitude and phase of the $\mathbf{E}$ and $\mathbf{H}$ field in the frequencydomain for each grid point along the TFSF boundary using the wave number calculated in step 3.

5. Calculate the time-domain $\mathbf{E}$ and $\mathbf{H}$ fields (using an inverse Fast Fourier Transform) for each grid point along the TFSF boundary.

6. Use the calculated time-domain $\mathbf{E}$ and $\mathbf{H}$ to correct for the fields along the TFSF boundary.

The utility of this method allows the TFSF boundary corrections to be computed a priori and stored in memory for use during the FDTD simulation. This allows for the TFSF boundary corrections to be computed once, but used multiple times (i.e., as would be needed for a Monte-Carlo simulation of a rough surface). 
Also, the AFP method does not restrict the calculation to just a homogenous media. It is easy to add a dielectric (or metal) interface to the calculation of the magnitude and phase of the $\mathbf{E}$ and $\mathbf{H}$ field in the frequency-domain before the inverse Fast Fourier Transform [39]. The only additional step required is to calculate the frequency dependent reflection and transmission coefficients caused by the dielectric (or metal) interface. The other media (i.e., a dielectric) need not be simple. It can be lossy. For the $\mathrm{TM}_{z}$ case outlined in this section, the calculations for the wave numbers $\left(k_{x, y}\right)$, the reflection coefficient $(\Gamma)$ and transmission coefficient $(T)$ are given for a horizontal interface as [39]:

$$
\begin{gathered}
k_{x, \text { air }}=\frac{k_{\text {ratio }} 2 \pi \cos (\phi)}{S_{\text {air }}} \\
k_{y, \text { air }}=\frac{k_{\text {ratio }} 2 \pi \sin (\phi)}{S_{\text {air }}} \\
k_{x, \text { dielectric }}=\breve{k}_{x, \text { air }} \\
k_{y, \text { dielectric }}=\kappa_{y}^{\prime}+j \kappa^{\prime \prime}
\end{gathered}
$$

where $k_{\text {ratio }}$ is the solution to the dispersion equation (3.43). $k_{\text {ratio }}$ is how much to modify the wavenumber in air to account for the dispersion within the FDTD grid. $S_{a i r}$ is the Courant number for air, and $\kappa_{y}^{\prime}$ and $\kappa_{y}^{\prime \prime}$ are given by [42]

$$
\kappa_{y}^{\prime}=\frac{1}{2} \cos ^{-1}\left[\frac{U V}{\sqrt{8} C^{\prime}}\right]
$$




$$
\kappa_{y}^{\prime \prime}=-\frac{1}{2} \cosh ^{-1}\left[\frac{U}{\sqrt{2}}\right]
$$

where

$$
\begin{gathered}
U=\left(M+\sqrt{\left(M-2 C^{\prime}\right)\left(M+2 C^{\prime}\right)}\right)^{1 / 2} \\
V=M-\sqrt{\left(M-2 C^{\prime}\right)\left(M+2 C^{\prime}\right)} \\
M=1+C^{\prime 2}+C^{\prime \prime 2} \\
C^{\prime}=1+2\left[\sin ^{2}\left(\kappa_{x}\right)-\frac{\mu_{r} \epsilon_{r}}{S_{c}^{2}} \sin ^{2}\left(\frac{\omega \Delta_{t}}{2}\right)\right] \\
C^{\prime \prime}=-\frac{\mu_{r} \epsilon_{r}}{S_{c}^{2}} L_{\sigma} \sin \left(\omega \Delta_{t}\right)
\end{gathered}
$$

with $S_{c}^{2}$ being the Courant number for the dielectric layer and $L_{\sigma}$ being the loss factor defined as $\sigma \Delta_{t} /(2 \epsilon)$.

With the wave number defined for each region taking into account the dispersion of the FDTD grid, the reflection and transmission coefficients can be calculated as [42]:

$$
\begin{aligned}
\Gamma_{t m} & =\frac{\mu_{2} \sin \left(2 \kappa_{1 y}\right)-\mu_{1} \sin \left(2 \kappa_{2 y}\right)}{\mu_{2} \sin \left(2 \kappa_{1 y}\right)+\mu_{1} \sin \left(2 \kappa_{2 y}\right)} \\
T_{t m} & =\frac{2 \mu_{2} \sin \left(2 \kappa_{1 y}\right)}{\mu_{2} \sin \left(2 \kappa_{1 y}\right)+\mu_{1} \sin \left(2 \kappa_{2 y}\right)}
\end{aligned}
$$

With the reflection and transmission coefficients determined for the FDTD grid, including the dispersion of the grid, the frequency domain TFSF boundary corrections can be calculated following [39]. The time-domain TFSF boundary 
corrections can be calculated from the frequency domain TFSF boundary corrections using an inverse FFT (e.g., FFTw). This time-domain TFSF boundary correction can now be used in the standard TFSF updating equations to source a plane wave into a simulation space with a dielectric half-space interface.

The TFSF method outlined above does not yet address dispersive media, but is an excellent starting point for simulating plane waves incident upon dispersive media. This is because the method above works by calculating the wave number on a frequency point by frequency point basis for the TFSF updating equations based on a constant $\epsilon_{r}$ and $\sigma$. For dispersive media, the permittivity is complex, and changing with frequency. The imaginary part of the complex frequency dependent permittivity can be written as a frequency dependent conductivity using [20]

$$
\sigma(f)=-2 \pi f \epsilon_{r}^{\prime \prime}(f) \epsilon_{0},
$$

where $\sigma$ is the conductivity, $f$ is the frequency, $\epsilon_{r}^{\prime \prime}$ is the imaginary part of the complex permittivity, and $\epsilon_{0}$ is the permittivity of free space. The frequency dependent conductivity (along with the real part of the frequency dependent $\left.\epsilon_{r}\right)$ calculated using (3.57) is then used in equations (3.47) - (3.54) to correctly calculate the wave number of the dispersive media that is required for the TFSF updating equations. 


\subsection{Grid Truncation: The Perfectly Matched Layer}

The basic FDTD simulation using the Yee grid has been outlined in a prior section. Also discussed is how to source a plane-wave into the FDTD grid. Now, the discussion turns to what happens when the FDTD grid is truncated when the grid is entered into a computer.

When a FDTD space is simulated in a computer, the grid must have a finite size as there is no computer with an infinite amount of memory. Because of this, when doing the finite-differencing required by the FDTD grid the $\mathbf{E}$ field along the outer FDTD grid cells will have zero magnitude. This is the equivalent of putting the FDTD simulation in a perfectly conducting box. This boundary condition will cause any wave that reaches the edge of the simulation space to be reflected back towards the center of the FDTD grid. This is not always an issue. For example, if a waveguide or coaxial cable are to be simulated, then this default boundary condition would be accurate and valid. However, this becomes a problem when scattering problems (i.e., radar cross section simulations) are being calculated as the default FDTD boundary condition is not an open region. Two methods to address this problem are [34]:

1. Make the FDTD simulation grid large enough so that the scattered waves do not interact with the FDTD grid boundary. This is an inefficient way to handle the problem as the area of interest would typically be a small 


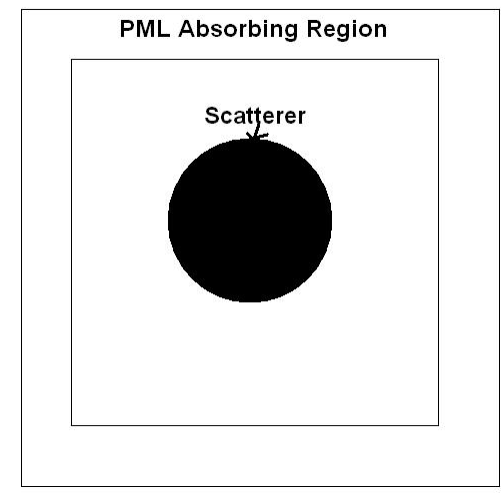

Figure 3.5: The FDTD grid showing where the absorbing PML is located.

portion of the FDTD grid.

2. Determine how to absorb the outgoing wave so there are no reflections at the boundary. This method is more efficient as the FDTD grid needs only to be large enough to encompass the scatterer only (illustrated in Figure 3.5).

Of the two methods listed above, number 2 is most often used. The first method to absorb the outgoing wave was developed in 1981 by Mur and was called an Absorbing Boundary Condition (ABC) [37]. This first ABC worked well for waves of normal incidence to the FDTD boundary, but not well for oblique angles of incidence. This limited the usefulness of the original ABCs.

In 1994 Berenger [43] introduced a new type of ABC, called a Perfectly Matched Layer (PML). In this pioneering work, Berenger derived a split-field formulation of Maxwell's equations where each vector field component is split into two orthogonal components. This leads to a set of 12 coupled differential 
equations (instead of the six used for the Yee grid). Then, by selecting the material parameters of the added PML layer (i.e. $\epsilon_{r}, \sigma, \mu_{r}$, and $\sigma^{*}$ ), an absorbing layer was constructed that would allow a wave to be transmitted from the FDTD grid to the PML grid without reflection. In the PML grid, the wave would attenuate, reflect off of the PML truncation and attenuate further before being introduced back into the main FDTD grid. The consequence of this attenuation was to reduce the level of the FDTD grid truncation reflection by many orders of magnitude; making the reflected wave negligible. The PML is the computational equivalent to the walls in an anechoic chamber.

Since the introduction of the PML by Berenger in 1994 [43], several advances have been made to improve upon the accuracy and usability of the PML. In 1995 Sacks [44] and in 1996 Gedney [45] introduced the uniaxial PML (UPML) which combined the split fields of Berenger's PML by creating a permittivity and permeability tensor for the PML layers. With a uniaxial material for the PML, the PML layer became physical since the fields no longer need to be split up. However, the UPML can suffer from large reflection for highly evanescent waves or from late-time, low-frequency, interactions [34].

Another advance in PML theory came in 2000 when Roden and Gedney introduced the Convolutional PML (CPML) [46]. This paper introduced the concept of a complex frequency-shifted (CFS) tensor to overcome the late-time reflections. The CPML overcame the initial UPML issue of highly evanescent 
waves and late-time interactions. However, the CFS has also be applied to the UPML, which eliminated the late-time reflection problem of the original UPML work [34].

A survey of commercially available FDTD software reveals the UPML to be the most common form of PML. Because of this, and the ease of implementing the UPML with dispersive, inhomogeneous, and bi-axial material, it is selected for this work [34].

\subsubsection{Implementation of UPML in FDTD}

To implement the UPML material in the FDTD grid, a new methodology for updating the $\mathbf{E}$ and $\mathbf{H}$ must be used as the UPML layer is both dispersive and anisotropic. This new methodology is called the auxiliary differential equation (ADE) method [34]. Utilizing the complex frequency shifted (CFS) stretchedcoordinate form of implementing the PML, the time-dependent Ampere's law and Faraday's law can be expressed in the frequency domain as [34]:

$$
\left[\begin{array}{c}
\frac{\partial \breve{H}_{z}}{\partial \breve{H}_{y}}-\frac{\partial \breve{H}_{y}}{\partial \breve{H}_{z}} \\
\frac{\partial \breve{H}_{x}}{\partial \breve{H}_{z}}-\frac{\partial \breve{H}_{z}}{\partial \breve{H}_{x}} \\
\frac{\partial \breve{H}_{y}}{\partial \breve{H}_{x}}-\frac{\partial \breve{H}_{x}}{\partial \breve{H}_{y}}
\end{array}\right]=j \omega \epsilon\left[\begin{array}{ccc}
\frac{s_{y} s_{z}}{s_{x}} & 0 & 0 \\
0 & \frac{s_{x} s_{z}}{s_{y}} & 0 \\
0 & 0 & \frac{s_{x} s_{y}}{s_{z}}
\end{array}\right]\left[\begin{array}{c}
\breve{E}_{x} \\
\breve{E}_{y} \\
\breve{E}_{z}
\end{array}\right]
$$

where $\breve{E}_{x}, \breve{E}_{y}, \breve{E}_{z}, \breve{H}_{x}, \breve{H}_{y}$, and $\breve{H}_{z}$ are the frequency domain electric and magnetic field components. For the derivation here the fields are in the frequency domain, 
therefore the $\mathrm{E}$ and $\mathrm{H}$ field components are labeled with a breve above them to separate them from the time-domain field quantities. The terms $s_{x}, s_{y}$, and $s_{z}$ are the coordinate stretching terms given by:

$$
\begin{aligned}
& s_{x}=\kappa_{x, U}+\frac{\sigma_{x, U}}{j \omega \epsilon_{0}} \\
& s_{y}=\kappa_{y, U}+\frac{\sigma_{y, U}}{j \omega \epsilon_{0}} \\
& s_{z}=\kappa_{z, U}+\frac{\sigma_{z, U}}{j \omega \epsilon_{0}}
\end{aligned}
$$

In equations (3.59)- (3.61) the term $\sigma_{x, U}$ (or $\sigma_{y, U}$ or $\sigma_{z, U}$ ) and $\kappa_{x, U}$ (or $\kappa_{y, U}$ or $\kappa_{z, U}$ ) represent the loss of the PML layer and is graded based on [34]

$$
\begin{gathered}
\sigma_{x, U}(x)=\frac{x}{d} \sigma_{x, \max } \\
\kappa_{x, U}(x)=1+\left(\kappa_{x, \max }-1\right)\left(\frac{x}{d}\right)^{m}
\end{gathered}
$$

where $x$ is the position in the PML and $d$ is the thickness of the PML layer and $\mathrm{m}=4$ [34]. Also $\kappa_{x, \max }=1$ and $\sigma_{x, \max }$ is given by:

$$
\sigma_{x, \max }=-\frac{(m+1) \ln [R(0)]}{2 \eta d}
$$

where $\eta$ is the free-space impedance, $\mathrm{R}(0)$ is the desired reflection error (typically set to $1 \times 10^{-7}$ ), and $d$ is the thickness of the PML layer. 
Returning to equation (3.58) and substituting equations (3.59) - (3.61) in would lead to a convolution between the coordinate stretching terms and the Efield. As the convolution would be a very computationally intense operation, it is more efficient to decouple the frequency dependent terms by defining the electric flux density $(D)$ in terms of the electric field intensity $(E)$ and the coordinate stretching terms using $[45,47]$ :

$$
\begin{aligned}
& \breve{D}_{x}=\epsilon \frac{s_{z}}{s_{x}} \breve{E}_{x} \\
& \breve{D}_{y}=\epsilon \frac{s_{x}}{s_{y}} \breve{E}_{y} \\
& \breve{D}_{y}=\epsilon \frac{s_{y}}{s_{z}} \breve{E}_{z}
\end{aligned}
$$

Plugging in equations (3.65)- (3.67) into equation (3.58) gives

$$
\left[\begin{array}{c}
\frac{\partial \breve{H}_{z}}{\partial \breve{H}_{y}}-\frac{\partial \breve{H}_{y}}{\partial \breve{H}_{z}} \\
\frac{\partial \breve{H}_{x}}{\partial \breve{H}_{z}}-\frac{\partial \breve{H}_{z}}{\partial \breve{H}_{x}} \\
\frac{\partial \breve{H}_{y}}{\partial \breve{H}_{x}}-\frac{\partial \breve{H}_{x}}{\partial \breve{H}_{y}}
\end{array}\right]=j \omega\left[\begin{array}{ccc}
s_{y} & 0 & 0 \\
0 & s_{z} & 0 \\
0 & 0 & s_{x}
\end{array}\right]\left[\begin{array}{c}
\breve{D}_{x} \\
\breve{D}_{y} \\
\breve{D}_{z}
\end{array}\right]
$$

Substitute equation (3.59)- (3.61) into equation (3.68) and apply the inverse Fourier transform using the identity $j \omega f(\omega) \rightarrow(\partial / \partial t) f(t)$. This gives 


$$
\begin{aligned}
{\left[\begin{array}{c}
\frac{\partial H_{z}}{\partial H_{y}}-\frac{\partial H_{y}}{\partial H_{z}} \\
\frac{\partial H_{x}}{\partial H_{z}}-\frac{\partial H_{z}}{\partial H_{x}} \\
\frac{\partial H_{y}}{\partial H_{x}}-\frac{\partial H_{x}}{\partial H_{y}}
\end{array}\right]=\frac{\partial}{\partial t}\left[\begin{array}{ccc}
\kappa_{y, U} & 0 & 0 \\
0 & \kappa_{z, U} & 0 \\
0 & 0 & \kappa_{x, U}
\end{array}\right]\left[\begin{array}{c}
D_{x} \\
D_{y} \\
D_{z}
\end{array}\right] } \\
+\frac{1}{\epsilon_{0}}\left[\begin{array}{ccc}
\sigma_{y, U} & 0 & 0 \\
0 & \sigma_{z, U} & 0 \\
0 & 0 & \sigma_{x, U}
\end{array}\right]\left[\begin{array}{c}
D_{x} \\
D_{y} \\
D_{z}
\end{array}\right]
\end{aligned}
$$

Equation (3.69) can be discretized in the standard Yee lattice. The normal leapfrogging described earlier in this chapter will work with the loss terms in the PML layer averaged in time according to the semi-implicit scheme [34]. The discrete expression for $D_{x}$ (with similar equations for $D_{y}$ and $D_{z}$ ) is:

$$
\begin{aligned}
\left.D_{x}\right|_{i+1 / 2, j, k} ^{n+1}= & \\
& \left.\left.C_{1}\right|_{i+1 / 2, j, k} D_{x}\right|_{i+1 / 2, j, k} ^{n}+\left.C_{2}\right|_{i+1 / 2, j, k} \\
& \left(\frac{\left.H_{z}\right|_{i+1 / 2, j+1 / 2, k} ^{n+1 / 2}-\left.H_{z}\right|_{i+1 / 2, j-1 / 2, k} ^{n+1 / 2}}{\Delta y}-\right. \\
& \left.\frac{\left.H_{y}\right|_{i+1 / 2, j, k+1 / 2} ^{n+1 / 2}-\left.H_{y}\right|_{i+1 / 2, j, k-1 / 2} ^{n+1 / 2}}{\Delta z}\right)
\end{aligned}
$$


with the updating coefficients $C_{1}$ and $C_{2}$ defined as [34]:

$$
\begin{aligned}
& C_{1}=\frac{2 \epsilon_{0} \kappa_{y, U}-\sigma_{y, U} \Delta t}{2 \epsilon_{0} \kappa_{y, U}+\sigma_{y, U} \Delta t} \\
& C_{2}=\frac{2 \epsilon_{0} \Delta t}{2 \epsilon_{0} \kappa_{y, U}+\sigma_{y, U} \Delta t}
\end{aligned}
$$

Equations (3.71) and (3.72) are similar to $C_{a}$ and $C_{b}$ in equations (3.25) and (3.26), except $C_{1}$ and $C_{2}$ are modified to allow for the loss of the UPML layer.

Equation (3.70) is used to step the $\mathbf{D}$ field, but the $\mathbf{E}$ field is needed to update the magnetic fields, so $\mathbf{D}$ must be converted to $\mathbf{E}$ by substituting (3.59) - (3.61) into equations (3.65) - (3.67) to get:

$$
\begin{aligned}
& \left(\kappa_{x, U}+\frac{\sigma_{x, U}}{j \omega \epsilon_{0}}\right) \breve{D}_{x}=\epsilon\left(\kappa_{z, U}+\frac{\sigma_{z, U}}{j \omega \epsilon_{0}}\right) \breve{E}_{x} \\
& \left(\kappa_{y, U}+\frac{\sigma_{y, U}}{j \omega \epsilon_{0}}\right) \breve{D}_{y}=\epsilon\left(\kappa_{x, U}+\frac{\sigma_{x, U}}{j \omega \epsilon_{0}}\right) \breve{E}_{y} \\
& \left(\kappa_{z, U}+\frac{\sigma_{z, U}}{j \omega \epsilon_{0}}\right) \breve{D}_{z}=\epsilon\left(\kappa_{y, U}+\frac{\sigma_{y, U}}{j \omega \epsilon_{0}}\right) \breve{E}_{z}
\end{aligned}
$$

Equations (3.73) - (3.75) can be converted to the time-domain by multiplying both sides by $j \omega$ and taking the inverse Fourier transform to give [34]: 


$$
\begin{gathered}
\frac{\partial}{\partial t}\left(\kappa_{x, U} D_{x}\right)+\frac{\sigma_{x, U}}{\epsilon_{0}} D_{x}=\epsilon\left[\frac{\partial}{\partial t}\left(\kappa_{z, U} E_{x}+\frac{\sigma_{z, U}}{\epsilon_{0}} E_{x}\right)\right] \\
\frac{\partial}{\partial t}\left(\kappa_{y, U} D_{y}\right)+\frac{\sigma_{y, U}}{\epsilon_{0}} D_{y}=\epsilon\left[\frac{\partial}{\partial t}\left(\kappa_{x, U} E_{y}+\frac{\sigma_{x, U}}{\epsilon_{0}} E_{y}\right)\right] \\
\frac{\partial}{\partial t}\left(\kappa_{z, U} D_{z}\right)+\frac{\sigma_{z, U}}{\epsilon_{0}} D_{z}=\epsilon\left[\frac{\partial}{\partial t}\left(\kappa_{y, U} E_{z}+\frac{\sigma_{y, U}}{\epsilon_{0}} E_{z}\right)\right]
\end{gathered}
$$

Equations (3.76) - (3.78) can be discretized using the standard Yee grid and by time averaging the loss terms using the semi-implicit scheme. Doing this will give the following equation for $\mathrm{E}_{x}$ (with similar for $\mathrm{E}_{y}$ and $\mathrm{E}_{z}$ ) [34]:

$$
\begin{aligned}
& \left.E_{x}\right|_{i+1 / 2, j, k} ^{n+1}=\left.\left.C_{3}\right|_{i+1 / 2, j, k} E_{x}\right|_{i+1 / 2, j, k} ^{n}+\left.C_{4}\right|_{i+1 / 2, j, k} \cdot \\
& \quad\left[\left.\left.C_{5}\right|_{i+1 / 2, j, k} \cdot D_{x}\right|_{i+1 / 2, j, k} ^{n+1}-\left.\left.C_{6}\right|_{i+1 / 2, j, k} \cdot D_{x}\right|_{i+1 / 2, j, k} ^{n}\right]
\end{aligned}
$$

where the updating coefficients $C_{3}$ to $C_{6}$ are given by

$$
\begin{gathered}
C_{3}=\frac{2 \epsilon_{0} \kappa_{z, U}-\sigma_{z, U} \Delta t}{2 \epsilon_{0} \kappa_{z, U}+\sigma_{z, U} \Delta t} \\
C_{4}=\frac{1}{\left(2 \epsilon_{0} \kappa_{z, U}+\sigma_{z, U} \Delta t\right) \epsilon}
\end{gathered}
$$




$$
\begin{aligned}
& C_{5}=2 \epsilon_{0} \kappa_{x, U}+\sigma_{x, U} \Delta t \\
& C_{6}=2 \epsilon_{0} \kappa_{x, U}-\sigma_{x, U} \Delta t
\end{aligned}
$$

So, to update $\mathbf{E}$ a two-step process is needed. First, update $\mathbf{D}$ according to equation (3.70) and then update $\mathbf{E}$ according to equation (3.79).

A similar two-step procedure is needed for $\mathbf{H}$ in the grid. Starting with Faraday's Law in the stretched coordinate frequency domain form as [34]:

$$
\left[\begin{array}{c}
\frac{\partial \breve{E}_{z}}{\partial \breve{E}_{y}}-\frac{\partial \breve{E}_{y}}{\partial \breve{E}_{z}} \\
\frac{\partial \breve{E}_{x}}{\partial \breve{E}_{z}}-\frac{\partial \breve{E}_{z}}{\partial \breve{E}_{x}} \\
\frac{\partial \breve{E}_{y}}{\partial \breve{E}_{x}}-\frac{\partial \breve{E}_{x}}{\partial \breve{E}_{y}}
\end{array}\right]=-j \omega \mu\left[\begin{array}{ccc}
\frac{s_{y} s_{z}}{s_{x}} & 0 & 0 \\
0 & \frac{s_{x} s_{z}}{s_{y}} & 0 \\
0 & 0 & \frac{s_{x} s_{y}}{s_{z}}
\end{array}\right]\left[\begin{array}{c}
\breve{H}_{x} \\
\breve{H}_{y} \\
\breve{H}_{z}
\end{array}\right]
$$

where $\breve{E}_{x}, \breve{E}_{y}, \breve{E}_{z}, \breve{H}_{x}, \breve{H}_{y}$, and $\breve{H}_{z}$ are the frequency domain electric and magnetic field components with $s_{x}, s_{y}$, and $s_{z}$ given by equations (3.59) - (3.61).

To decouple the frequency dependent terms, define the constitutive relationship between $\mathbf{B}$ and $\mathbf{H}$ as [34]

$$
\begin{aligned}
& \breve{B}_{x}=\epsilon \frac{s_{z}}{s_{x}} \breve{H}_{x} \\
& \breve{B}_{y}=\epsilon \frac{s_{x}}{s_{y}} \breve{H}_{y}
\end{aligned}
$$




$$
\breve{B}_{y}=\epsilon \frac{s_{y}}{s_{z}} \breve{H}_{z}
$$

With this, an identical procedure used to derive the $\mathrm{D}_{x}$ and $\mathrm{E}_{x}$ equations can be followed to give the updating equations for $\mathrm{B}_{x}$ and $\mathrm{H}_{x}[34]$

$$
\begin{aligned}
& \left.B_{x}\right|_{i, j+1 / 2, k+1 / 2} ^{n+3 / 2}= \\
& \left.\left.\quad D_{1}\right|_{i, j+1 / 2, k+1 / 2} B_{x}\right|_{i, j+1 / 2, k+1 / 2} ^{n+1 / 2}+\left.D_{2}\right|_{i, j+1 / 2, k+1 / 2} \cdot \\
& \quad\left(\frac{\left.E_{z}\right|_{i, j+1, k+1 / 2} ^{n+1}-\left.E_{z}\right|_{i, j, k+1 / 2} ^{n+1}}{\Delta y}-\frac{\left.E_{y}\right|_{i, j+1 / 2, k+1} ^{n+1}-\left.E_{y}\right|_{i, j+1 / 2, k} ^{n+1}}{\Delta z}\right)
\end{aligned}
$$

$$
\begin{aligned}
& \left.H_{x}\right|_{i, j+1 / 2, k+1 / 2} ^{n+3 / 2}=\left.\left.D_{3}\right|_{i, j+1 / 2, k+1 / 2} H_{x}\right|_{i, j+1 / 2, k+1 / 2} ^{n+1 / 2}+\left.D_{4}\right|_{i, j+1 / 2, k+1 / 2} \cdot \\
& \left.\quad\left[\left.\left.D_{5}\right|_{i, j+1 / 2, k+1 / 2} \cdot B_{x}\right|_{i, j+1 / 2, k+1 / 2} ^{n+3 / 2}-\left.\left.D_{6}\right|_{i, j+1 / 2, k+1 / 2} \cdot B_{x}\right|_{i, j+1 / 2, k+1 / 2} ^{n+1 / 2}\right]^{2}\right]^{2}
\end{aligned}
$$

where the terms $D_{1}$ to $D_{6}$ are the updating coefficients defined as

$$
\begin{aligned}
& D_{1}=\frac{2 \epsilon_{0} \kappa_{y, U}-\sigma_{y, U} \Delta t}{2 \epsilon_{0} \kappa_{y, U}+\sigma_{y, U} \Delta t} \\
& D_{2}=\frac{2 \epsilon_{0} \Delta t}{2 \epsilon_{0} \kappa_{y, U}+\sigma_{y, U} \Delta t}
\end{aligned}
$$




$$
\begin{gathered}
D_{3}=\frac{2 \epsilon_{0} \kappa_{z, U}-\sigma_{z, U} \Delta t}{2 \epsilon_{0} \kappa_{z, U}+\sigma_{z, U} \Delta t} \\
D_{4}=\frac{1}{\left(2 \epsilon_{0} \kappa_{z, U}+\sigma_{z, U} \Delta t\right) \mu} \\
D_{5}=2 \epsilon_{0} \kappa_{x, U}+\sigma_{x, U} \Delta t \\
D_{6}=2 \epsilon_{0} \kappa_{x, U}-\sigma_{x, U} \Delta t
\end{gathered}
$$

For the updating coefficients listed in equations (3.71) - (3.72) and (3.80) - (3.83) and equations (3.90) - (3.95) set $\sigma_{x-z, U}=0$ and set $\kappa_{x-z, U}=1$ for the main FDTD grid. In the UPML regions, vary $\sigma_{x-z, U}$ as given by equation (3.62) and vary $\kappa_{x-z, U}$ as given by equation (3.63).

\subsection{Near-to-Far-Field Transformation}

FDTD simulations are inherently near field simulations. To get the far-field scattering response (or antenna radiation pattern), either the FDTD grid must be large enough that the scattered waves propagate far enough that the response is far-field or equivalent surface currents (e.g., Huygen's Principle [20]) can be used to calculate the far-field radiation from the near-field FDTD simulation data. The first method (the large FDTD grid) is not desirable since the large grid will have a large computational load and the wave will suffer from numerical artifacts in the FDTD grid [39]. The second method, called the Near-to-FarField (NTFF) Transformation has a smaller computational load and will not 
suffer from the same numerical artifacts. These benefits make the equivalent surface current method of the NTFF Transformation the preferred choice for scattering simulations with the FDTD method [38].

It is easier to describe and illustrate the NTFF transformation in two dimensions, so the discussion below will be done for the two dimensional case of a $\mathrm{TM}_{z}$ FDTD simulation (i.e., only $\mathrm{E}_{z}, \mathrm{H}_{x}$ and $\mathrm{H}_{y}$ field components, with the frequency domain phasors represented as $\breve{E}_{z}, \breve{H}_{x}$ and $\breve{H}_{y}$ ). Assume that an arbitrary scattering structure can be enclosed by an arbitrary contour, $C_{i}$ having the unit normal pointing outward (labeled $\hat{\mathbf{n}}_{\mathbf{a}}$ in Figure 3.6). Next, assume that an arbitrary contour exists at infinity, $C_{\infty}$, which is centered on the same coordinate system as the $C_{i}$ contour that has a normal directed outward $\hat{\mathbf{n}}_{\infty}=\hat{\mathbf{r}}$. Finally, assume that the two contours are connected with an infinitely thin "umbilical" path to form a single closed, continuous path [34].

By Green's theorem [48] applied to the scalar frequency-domain function of $\breve{E}_{z}(\mathbf{r})$ and $G\left(r \mid r^{\prime}\right)$ :

$$
\begin{aligned}
d \int_{S} s^{\prime}\left[\breve{E}_{z}\left(\mathbf{r}^{\prime}\right)\left(\nabla^{2}\right) G\left(\mathbf{r} \mid \mathbf{r}^{\prime}\right)-G\left(\mathbf{r} \mid \mathbf{r}^{\prime}\right)\left(\nabla^{2}\right) \breve{E}_{z}\left(\mathbf{r}^{\prime}\right)\right] \\
=\oint_{C_{\infty}}\left[\breve{E}_{z}\left(\mathbf{r}^{\prime}\right) \frac{\partial G\left(\mathbf{r} \mid \mathbf{r}^{\prime}\right)}{\partial r^{\prime}}-G\left(\mathbf{r} \mid \mathbf{r}^{\prime}\right) \frac{\partial \breve{E}_{z}\left(\mathbf{r}^{\prime}\right)}{\partial r^{\prime}}\right] d C^{\prime} \\
-\oint_{C_{i}}\left[\breve{E}_{z}\left(\mathbf{r}^{\prime}\right) \frac{\partial G\left(\mathbf{r} \mid \mathbf{r}^{\prime}\right)}{\partial n_{a}^{\prime}}-G\left(\mathbf{r} \mid \mathbf{r}^{\prime}\right) \frac{\partial \breve{E}_{z}\left(\mathbf{r}^{\prime}\right)}{\partial n_{a}^{\prime}}\right] d C^{\prime}
\end{aligned}
$$




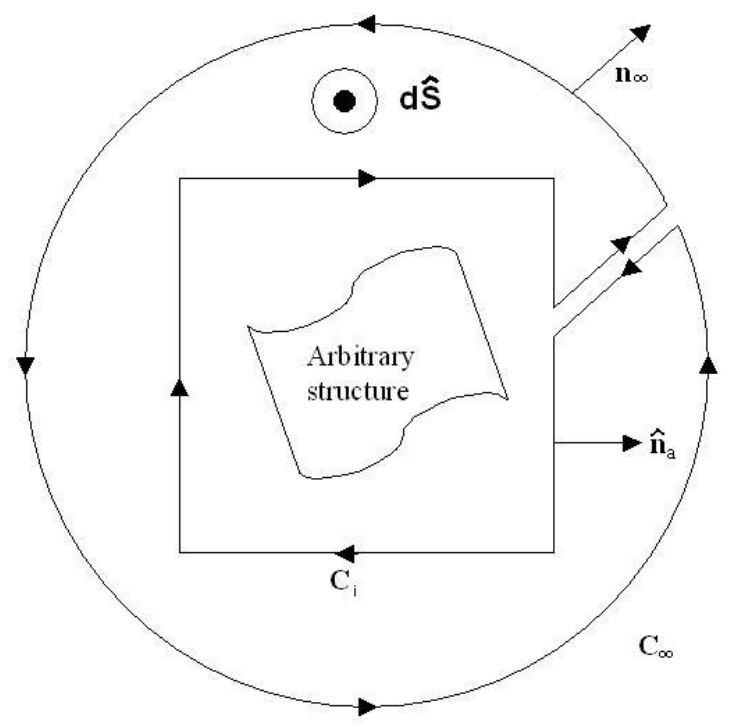

Figure 3.6: Showing a FDTD grid with a total field region and scattered field region.

where $\mathbf{r}$ is an observation point in the two-dimensional space, $\mathbf{r}^{\prime}$ is a source point and $d C^{\prime}$ is a differential path element along the combined $C_{i}$ and $C_{\infty}$ source contours. The negative sign in the right side of equation (3.96) results from the opposite orientations of $\hat{\mathbf{n}}_{\infty}=\hat{\mathbf{r}}$ and $\hat{\mathbf{n}}_{\mathbf{a}}$ relative to $\mathrm{S}$.

It can be shown that the $C_{\infty}$ goes to zero in the limit of $r^{\prime} \rightarrow \infty$ [34]. Further, from the definition of the Green function for time-harmonic systems [34]:

$$
\left(\nabla^{2}\right) G\left(\mathbf{r} \mid \mathbf{r}^{\prime}\right)=\delta\left(\mathbf{r}-\mathbf{r}^{\prime}\right)-k^{2} G\left(\mathbf{r} \mid \mathbf{r}^{\prime}\right)
$$

where $\delta$ is the Dirac delta function and $k$ is the wavenumber. The Helmholtz 
wave equation gives [20]:

$$
\left(\nabla^{2}\right)^{\prime} \breve{E}_{z}\left(\mathbf{r}^{\prime}\right)=-k^{2} \breve{E}_{z}\left(\mathbf{r}^{\prime}\right)
$$

Substituting equations (3.98) and (3.97) into the left side of equation (3.96) gives:

$$
\int_{S} \breve{E}_{z}\left(\mathbf{r}^{\prime}\right) \cdot \delta\left(\mathbf{r}-\mathbf{r}^{\prime}\right) d s^{\prime}=\breve{E}_{z}(\mathbf{r})
$$

Therefore equation (3.96) reduces to:

$$
\breve{E}_{z}(\mathbf{r})=\oint_{C_{i}}\left[G\left(\mathbf{r} \mid \mathbf{r}^{\prime}\right) \hat{\mathbf{n}}_{a}^{\prime} \cdot \nabla^{\prime} \breve{E}_{z}\left(\mathbf{r}^{\prime}\right)-\breve{E}_{z}\left(\mathbf{r}^{\prime}\right) \cdot \nabla^{\prime} G\left(\mathbf{r} \mid \mathbf{r}^{\prime}\right)\right]
$$

In two dimensions, $G\left(\mathbf{r} \mid \mathbf{r}^{\prime}\right)$ is given by the Hankel function [34]:

$$
G\left(\mathbf{r} \mid \mathbf{r}^{\prime}\right)=\frac{1}{4} H_{0}^{(2)}\left(k\left|\mathbf{r}-\mathbf{r}^{\prime}\right|\right)
$$

Looking at Figure 3.7, which shows an observation point $(\mathbf{P}=\mathbf{r})$ which is many wavelengths away (i.e., it is in the far-field) from the source contour of $C_{i}$. For an observation point clearly in the far-field, the value $k\left|\mathbf{r}-\mathbf{r}^{\prime}\right|$ is very large. In this case, it can be shown that the limiting expression for $G\left(\mathbf{r} \mid \mathbf{r}^{\prime}\right)$ is:

$$
\lim _{k\left|\mathbf{r}-\mathbf{r}^{\prime}\right| \rightarrow \infty} G\left(\mathbf{r} \mid \mathbf{r}^{\prime}\right)=\frac{j^{3 / 2}}{\sqrt{8 \pi k}} \frac{e^{-j k\left|\mathbf{r}-\mathbf{r}^{\prime}\right|}}{\left|\mathbf{r}-\mathbf{r}^{\prime}\right|^{1 / 2}}
$$




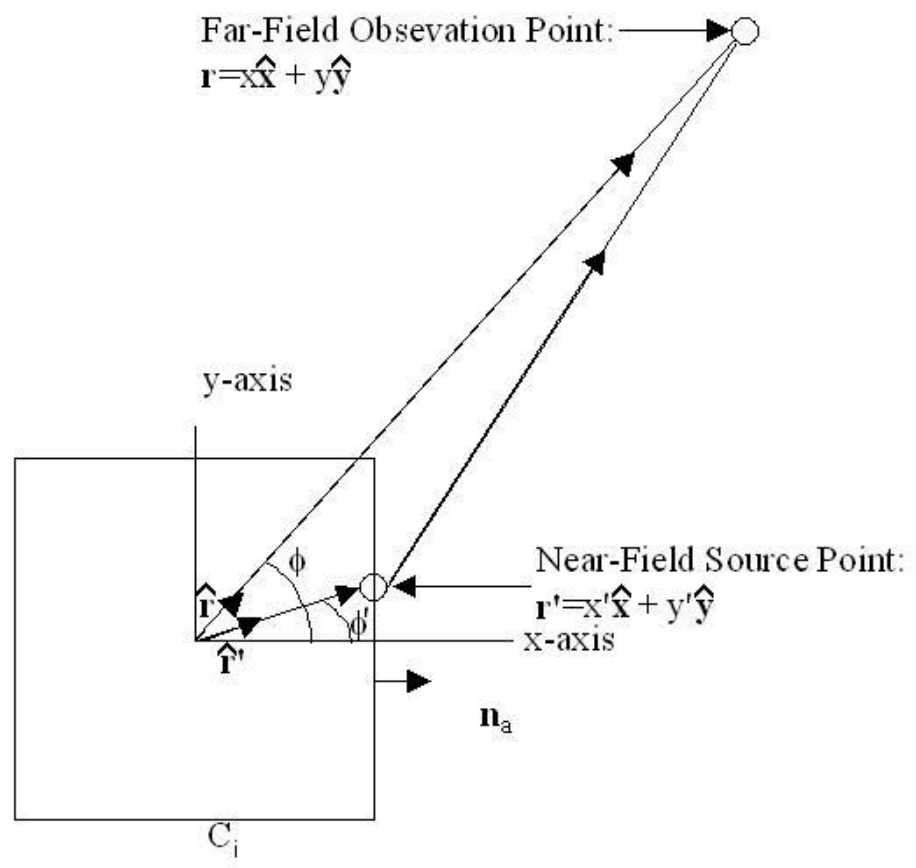

Figure 3.7: Far-Field observation point relative to the near-field integration boundary.

Applying the law of cosines to $\left|\mathbf{r}-\mathbf{r}^{\prime}\right|^{2}$, taking the square-root and expanding the result in a one-term binomial expansion (assuming $\left|\mathbf{r}^{\prime}\right| /|\mathbf{r}| \ll 1$ ), it can be shown that:

$$
\left|\mathbf{r}-\mathbf{r}^{\prime}\right| \cong r-r^{\prime} \cos \left(\phi-\phi^{\prime}\right)
$$

and

$$
\left|\mathbf{r}-\mathbf{r}^{\prime}\right|^{1 / 2} \cong r^{1 / 2}
$$

Substituting equations (3.103) and (3.104) into equation (3.102) gives the 
approximate answer to:

$$
\lim _{k\left|\mathbf{r}-\mathbf{r}^{\prime}\right| \rightarrow \infty} G\left(\mathbf{r} \mid \mathbf{r}^{\prime}\right)=\frac{j^{3 / 2}}{\sqrt{8 \pi k r}} e^{-j k\left[r-r^{\prime} \cos \left(\phi-\phi^{\prime}\right)\right]}=\frac{j^{3 / 2}}{\sqrt{8 \pi k r}} e^{-j k r} e^{+j k \hat{\mathbf{r}} \cdot \mathbf{r}^{\prime}}
$$

which gives

$$
\lim _{k\left|\mathbf{r}-\mathbf{r}^{\prime}\right| \rightarrow \infty} \nabla^{\prime} G\left(\mathbf{r} \mid \mathbf{r}^{\prime}\right)=(j k \hat{\mathbf{r}}) \frac{j^{3 / 2}}{\sqrt{8 \pi k r}} e^{-j k\left[r-r^{\prime} \cos \left(\phi-\phi^{\prime}\right)\right]}
$$

Substituting equations (3.105) and )3.106) into equation (3.100) gives the far-field electric field $\breve{E}_{z}(\mathbf{r})$ as:

$$
\lim _{k\left|\mathbf{r}-\mathbf{r}^{\prime}\right| \rightarrow \infty} \breve{E}_{z}(\mathbf{r})=\frac{e^{j 3 \pi / 4}}{\sqrt{8 \pi k r}} e_{-j k r} \oint_{C_{i}}\left[\hat{\mathbf{n}}_{a}^{\prime} \cdot \nabla \breve{E}_{z}\left(\mathbf{r}^{\prime}\right)-j k \breve{E}_{z}\left(\mathbf{r}^{\prime}\right) \hat{\mathbf{n}}_{a}^{\prime} \cdot \hat{\mathbf{r}}\right] e^{+j k \hat{\mathbf{r}} \cdot \mathbf{r}^{\prime}} d C^{\prime}
$$

For the two-dimensional case, the NTFF derivation can be completed by starting with:

$$
\nabla^{\prime} \breve{E}_{z}\left(\mathbf{r}^{\prime}\right)=\hat{\mathbf{x}}^{\prime} \frac{\partial \breve{E}_{z}}{\partial x^{\prime}}+\hat{\mathbf{y}}^{\prime} \frac{\partial \breve{E}_{z}}{\partial y^{\prime}}
$$

Replacing the the partial derivatives with respect to $x$ and $y$ with the corresponding magnetic fields in equation (3.108) gives:

$$
\nabla^{\prime} \breve{E}_{z}\left(\mathbf{r}^{\prime}\right)=\hat{\mathbf{x}}^{\prime}\left(-j \omega \mu_{0} \breve{H}_{y}\right)+\hat{\mathbf{y}}^{\prime}\left(j \omega \mu_{0} \breve{H}_{x}\right)=j \omega \mu_{0} \hat{\mathbf{z}}^{\prime} \times \breve{\mathbf{H}}\left(\mathbf{r}^{\prime}\right)
$$


Dotting $\hat{\mathbf{n}}_{\mathbf{a}}^{\prime}$ with equation (3.109) gives:

$$
\hat{\mathbf{n}}_{\mathbf{a}}^{\prime} \cdot \nabla^{\prime} \breve{E}_{z}\left(\mathbf{r}^{\prime}\right)=j \omega \mu_{0} \hat{\mathbf{n}}_{\mathbf{a}}^{\prime} \cdot\left[\hat{\mathbf{z}}^{\prime} \times \breve{\mathbf{H}}\left(\mathbf{r}^{\prime}\right)\right]=-j \omega \mu_{0} \hat{\mathbf{z}}^{\prime} \cdot\left[\hat{\mathbf{n}}_{\mathbf{a}}^{\prime} \times \breve{\mathbf{H}}\left(\mathbf{r}^{\prime}\right)\right]
$$

Using a vector identity equation (3.110) can be written as [34]:

$$
\breve{E}_{z}\left(\mathbf{r}^{\prime}\right) \hat{\mathbf{n}}_{\mathbf{a}}^{\prime} \cdot \hat{\mathbf{r}}=\left(\hat{\mathbf{z}} \times\left[\hat{\mathbf{n}}_{\mathbf{a}}^{\prime} \times \breve{\mathbf{E}}_{\mathbf{z}}\left(\mathbf{r}^{\prime}\right)\right]\right) \cdot \hat{\mathbf{r}}=\hat{\mathbf{n}}_{\mathbf{a}}^{\prime} \underbrace{(\hat{\mathbf{z}} \cdot \mathbf{E})}_{\breve{E}_{z}} \cdot \hat{\mathbf{r}}-\underbrace{\breve{\mathbf{E}}\left(\hat{\mathbf{z}} \cdot \hat{\mathbf{n}}_{\mathbf{a}}^{\prime}\right)}_{=\text {ohere }} \cdot \hat{\mathbf{r}}
$$

Using equations (3.110) and (3.111) in equation (3.107) gives the NTFF transformation in standard form as [34]

$$
\lim _{k\left|\mathbf{r}-\mathbf{r}^{\prime}\right| \rightarrow \infty} \breve{E}_{z}(\mathbf{r})=\underbrace{\frac{e^{-j k r}}{\sqrt{r}}}_{\text {PhaseTerm }} \frac{e^{j(\pi / 4)}}{\sqrt{8 \pi k}} \oint_{C_{i}}\left[\omega \mu_{0} \hat{\mathbf{z}}^{\prime} \cdot \breve{\mathbf{J}}_{\mathbf{e q}}\left(\mathbf{r}^{\prime}\right)-k \hat{\mathbf{z}}^{\prime} \times \breve{\mathbf{M}}_{\mathbf{e q}}\left(\mathbf{r}^{\prime}\right) \cdot \hat{\mathbf{r}}\right] e^{+j k \hat{\mathbf{r}} \cdot \mathbf{r}} d C^{\prime}
$$

where $\breve{\mathbf{J}}_{\mathbf{e q}}\left(\mathbf{r}^{\prime}\right)=\hat{\mathbf{n}}_{\mathbf{a}} \times \breve{\mathbf{H}}$ and $\breve{\mathbf{M}}_{\mathbf{e q}}\left(\mathbf{r}^{\prime}\right)=-\hat{\mathbf{n}}_{\mathbf{a}} \times \breve{\mathbf{E}}$ and are called the phasor equivalent electric and magnetic currents observed at $C_{i}$. Equation (3.112) can be solved with a numerical technique such as Simpson's rule or the trapezoidal rule. The magnitude of equation (3.112) can be written as:

$$
F(\phi)=\frac{e^{j(\pi / 4)}}{\sqrt{8 \pi k}} \oint_{C_{i}}\left[\omega \mu_{0} \hat{\mathbf{z}}^{\prime} \cdot \breve{\mathbf{J}}_{\mathbf{e q}}\left(\mathbf{r}^{\prime}\right)-k \hat{\mathbf{z}}^{\prime} \times \breve{\mathbf{M}}_{\mathbf{e q}}\left(\mathbf{r}^{\prime}\right) \cdot \hat{\mathbf{r}}\right] e^{+j k \hat{\mathbf{r}} \cdot \mathbf{r}} d C^{\prime}
$$


and this can be used to calculated the bistatic radar cross section (RCS) as [20]:

$$
R C S(\phi)=2 \pi \frac{|F(\phi)|^{2}}{\left|\breve{\mathbf{E}}_{\text {inc }}\right|^{2}}
$$

having the dimension of meters. In equation (3.114) $\breve{\mathbf{E}}_{\mathbf{i n c}}$ is the incident electric field.

$C_{i}$ is a virtual surface that is the locus points of space where the $E$ and $H$ field data are being compiled and integrated. Since $C_{i}$ can have an arbitrary shape, the shape can be assigned to lie along a rectangle in the scattered-field zone of a FDTD grid. Figure 3.8 shows a FDTD grid with the contour $C_{i}$ aligned with the $E_{z}$ grid points. The $H$ components are staggered one-half a grid cell to either side of the contour $C_{i}$. To address this issue with the offset $H$ fields, the geometric mean of the $H$ fields on either side of the contour $C_{i}$ is used as detailed in [49]. Because of the square-root being performed on the complex value, care must be taken when selected the root to ensure the correct phase angle is used for the NTFF transformation.

For a one-dimensional rough surface (as shown in Figure 3.9) the bistatic cross section is therefore given as [50]:

$$
\sigma\left(\theta_{s}, \theta_{i}\right)=2 \pi \frac{\left|E_{\text {scat }}\right|^{2}}{\left|E_{\text {inc }}\right|^{2} L}
$$

where $\theta_{s}$ is the angle of observation measured from the vertical, $\theta_{i}$ is the incident 


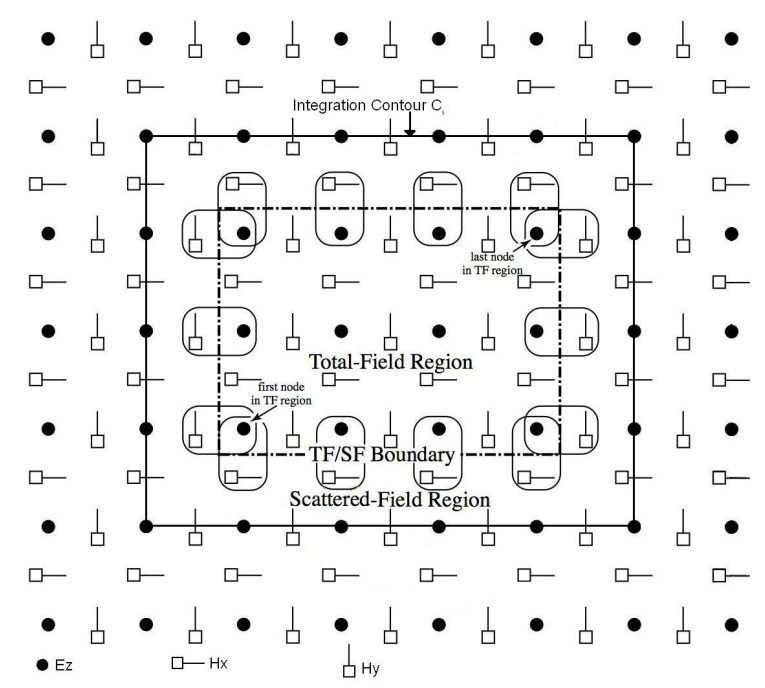

Figure 3.8: Integration contour, $C_{i}$ showing the $H$ field offset from the contour $C_{i}$.

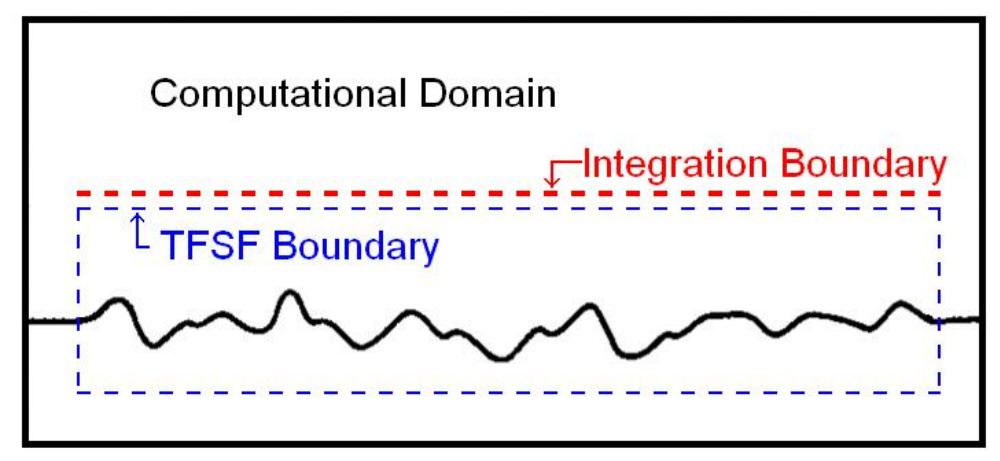

Figure 3.9: Integration contour for a one-dimensional rough surface. 
angle measured from the vertical, $E_{\text {scat }}$ is the scattered E-field (obtained using Equation (3.113)), $E_{i n c}$ is the incident E-field and $L$ is the length of the rough surface. It is worth noticing, the integration boundary for a one-dimensional rough surface is reduced to a single line as shown in Figure 3.9.

For such a one-dimensional rough surface simulation, the rough surface must be contained in the total-field region and the interface must be smooth in the scattered field region (as drawn in Figure 3.9). Also, to avoid spurious reflections at the TFSF interface, the rough surface cannot suddenly start. It must be slowly tapered (using a Gaussian profile) up to the normal rough surface height [50].

The FDTD simulations give the $E$ and $H$ fields in the time-domain, but the NTFF transformation in this section requires frequency domain data. To get the frequency-domain data from the time-domain data along the integration contour $C_{i}$ a discrete Fourier Transform (DFT) can be run concurrently with the FDTD simulation as detailed in [34].

\subsection{Dispersive Media}

As mentioned in the introduction, the quantum signature of the explosives will be modeled as a dispersive media. Until now, the simulations discussed in this dissertation has been for non-dispersive materials. The two main methods for simulating dispersive media in the FDTD grid are: 
1. The piecewise-linear recursive convolution (PLRC) method introduced by Luebbers et. al $[51,52,53]$.

2. The Auxiliary differential equation (ADE) method introduced by Kashiwa et. al. in 1990 [54] and Joseph et. al in 1991 [55]. This ADE method was made computationally efficient by Okoniewski et. al. [56] and reduced the amount of computational time required by the ADE method.

Because of the computational efficiency of the ADE method over the PLRC method, and because the UPML also used the ADE for updating the fields, the ADE method is selected for the work in this dissertation.

The ADE method models dispersive media in the FDTD grid by a set of auxiliary differential equations linking the polarization and the electric flux density $[54,55]$. These equations are stepped in time with the $E$ and $H$ fields in the FDTD grid.

\subsubsection{Lorentz Media}

There are three common models for dispersive material [20, 34]:

1. The Debye relaxation.

2. The Lorentzian resonance.

3. The Drude model of metals. 
Table 3.1: Lorentz model parameters for C-4 [10].

\begin{tabular}{|c|c|c|c|c|c|}
\hline Pole & $\epsilon_{\infty}$ & $\omega_{p}(\mathrm{rad} / \mathrm{sec})$ & $f_{p}(\mathrm{THz})$ & $\Delta \epsilon_{p}$ & $2 \delta_{p}(\mathrm{THz})$ \\
\hline 0 & 2.85 & - & - & - & - \\
\hline 1 & - & $5.07 \times 10^{12}$ & 0.808 & 0.263 & 1.376 \\
\hline 2 & - & $6.70 \times 10^{12}$ & 1.064 & 0.014 & 0.679 \\
\hline 3 & - & $8.52 \times 10^{12}$ & 1.356 & 0.034 & 1.188 \\
\hline 4 & - & $9.61 \times 10^{12}$ & 1.532 & 0.032 & 1.621 \\
\hline 5 & - & $1.24 \times 10^{13}$ & 1.980 & 0.050 & 2.036 \\
\hline 6 & - & $1.41 \times 10^{13}$ & 2.244 & 0.019 & 2.055 \\
\hline
\end{tabular}

The Lorentz model is used for modeling the dispersive dielectric constant of explosives in the terahertz frequency band as shown in [10] as it accurately captures the resonant molecular vibrations responsible for the changing dielectric constant. The Lorentz model is presented here [20, 34].

$$
\epsilon(\omega)=\epsilon_{\infty}+\sum_{p=1}^{P} \frac{\Delta \epsilon_{p} \omega_{p}^{2}}{\omega_{p}^{2}+2 j \omega \delta_{p}-\omega^{2}}
$$

where $\epsilon_{\infty}$ is the relative dielectric constant at infinite frequency, $\omega_{p}$ is the frequency of the pole, $\Delta \epsilon_{p}$ is the change in the relative permittivity due to the p'th pole pair and $\delta_{p}$ is the damping coefficient of the pole. An example frequency dependent dielectric constant of C-4 is detailed in Table 3.1 and displayed in Figure 3.10 [10].

The location of the Lorentz poles listed in Table 3.1 will be different than the locations of the absorption peaks in Table 2.3. In order to get the absorption 


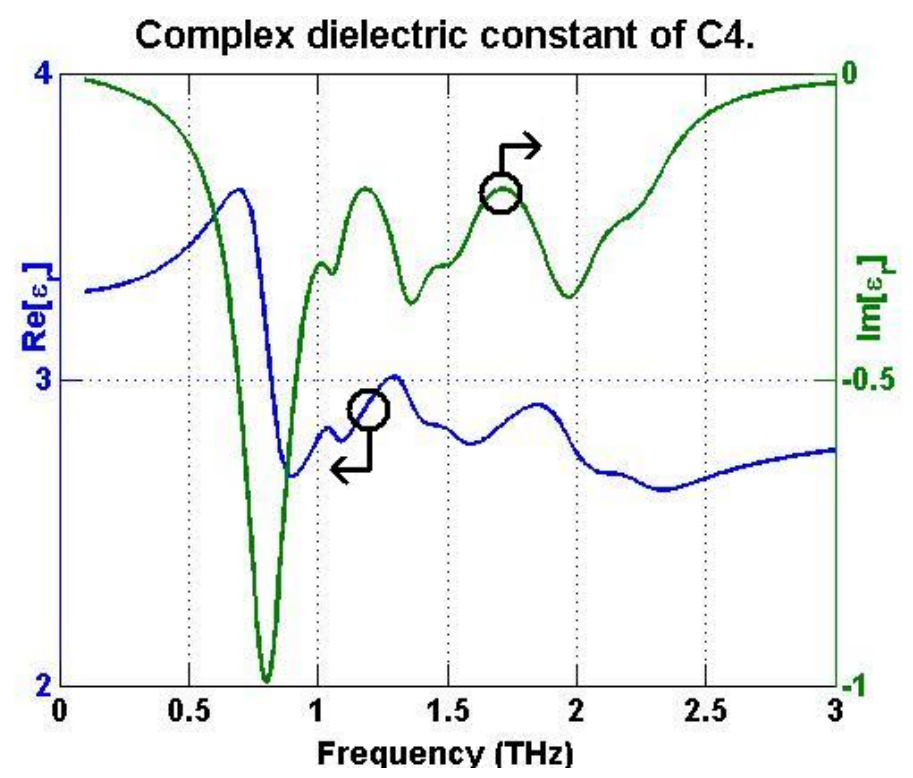

Figure 3.10: The complex frequency dependent dielectric constant of C-4. The left axis is the real part and the right axis is the imaginary part.

peaks in the location listed in Table 2.3, the Lorentz poles need to be located at the point on the curve were the slope is most rapidly changing (i.e. where the magnitude of the derivative is maximized). Since the magnitude of the derivative of any curve is not a maximum at a peak, the Lorentz poles will not be in the same location as the absorption peaks, but at a slightly higher frequency.

\subsubsection{Modify the FDTD update equations for the Lorentz Model.}

For a multipole Lorentz media, Ampere's law in the time domain is given by [34]

$$
\nabla \times \mathbf{H}=\epsilon_{0} \epsilon_{\infty} \frac{\partial \mathbf{E}}{\partial t}+\sigma \mathbf{E}+\sum_{p=1}^{P} \mathbf{J}_{\mathbf{p}}
$$

Using equation (3.116) to represent the frequency dependent permittivity of 
the media, a frequency dependent phasor polarization current for each pole is given by [34]

$$
\breve{\mathbf{J}}_{\mathbf{p}}=\epsilon_{o} \Delta \epsilon_{p}^{2} \omega_{p}^{2}\left(\frac{j \omega}{\omega_{p}^{2}+2 j \omega \delta_{p}-\omega^{2}}\right) \breve{\mathbf{E}}
$$

which can be rearranged and converted to the time-domain using the Fourier transform into

$$
\omega_{p}^{2} \mathbf{J}_{\mathbf{p}}+2 \delta_{p} \frac{\partial \mathbf{J}_{\mathbf{p}}}{\partial t}+\frac{\partial^{2} \mathbf{J}_{\mathbf{p}}}{\partial t^{2}}=\epsilon_{0} \Delta \epsilon_{p} \omega_{p}^{2} \frac{\partial \mathbf{E}}{\partial t}
$$

Equation (3.119) can be written in the same finite-difference manner as the $\mathbf{E}$ and $\mathbf{H}$ fields to allow for simultaneous updating for the polarization current $\left(\mathbf{J}_{\mathbf{p}}\right)$ using [34]

$$
\mathbf{J}_{p}^{n+1}=\alpha_{p} \mathbf{J}_{p}^{n}+\xi_{p} \mathbf{J}_{p}^{n-1}+\frac{\gamma_{p}}{2 \Delta t}\left(\mathbf{E}^{n+1}-\mathbf{E}^{n-1}\right)
$$

where $\alpha_{p}, \xi_{p}$ and $\gamma_{p}$ are given by:

$$
\begin{gathered}
\alpha_{p}=\frac{2-\omega_{p}^{2}(\Delta t)^{2}}{1+\delta_{p} \Delta t} \\
\xi_{p}=\frac{\delta_{p} \Delta t-1}{\delta_{p} \Delta t+1} \\
\gamma_{p}=\frac{\epsilon_{0} \Delta \epsilon_{p} \omega_{p}^{2}(\Delta t)^{2}}{1+\delta_{p} \Delta t}
\end{gathered}
$$

To update Ampere's law as given by equation (3.117) in the FDTD grid, 
equation (3.120) must be found at the $n+1 / 2$ time step, which can be found using the semi-implicit scheme as:

$$
\mathbf{J}_{p}^{n+1 / 2}=\frac{1}{2}\left(\mathbf{J}_{p}^{n}+\mathbf{J}_{p}^{n+1}\right)=\frac{1}{2}\left[\left(1+\alpha_{p}\right) \mathbf{J}_{p}^{n}+\xi_{p} \mathbf{J}_{p}^{n-1}+\frac{\gamma_{p}}{2 \Delta t}\left(\mathbf{E}^{n+1}-\mathbf{E}^{n-1}\right)\right]
$$

Using Equation (3.124) in Equation (3.117) and writing Ampere's law in the finite-difference form gives:

$$
\begin{aligned}
\nabla \times \mathbf{H}^{n+1 / 2}= & \epsilon_{0} \epsilon_{\infty}\left(\frac{\mathbf{E}^{n+1}-\mathbf{E}^{n}}{\Delta t}\right)+\sigma\left(\frac{\mathbf{E}^{n+1}-\mathbf{E}^{n}}{\Delta t}\right) \\
& \frac{1}{2} \sum_{p=1}^{P}\left[\left(1+\alpha_{p}\right) \mathbf{J}_{p}^{n}+\xi_{p} \mathbf{J}_{p}^{n-1}+\frac{\gamma_{p}}{2 \Delta t}\left(\mathbf{E}^{n+1}-\mathbf{E}^{n-1}\right)\right](3
\end{aligned}
$$

which can be rearranged for the FDTD E field updating as:

$$
\begin{aligned}
\mathbf{E}^{n+1}= & C_{1 d} \mathbf{E}^{n-1}+C_{2 d} \mathbf{E}^{n}+C_{3 d} \cdot \\
& \left(\nabla \times \mathbf{H}^{n+1 / 2}-\frac{1}{2} \sum_{p=1}^{P}\left[\left(1+\alpha_{p}\right) \mathbf{J}_{p}^{n}+\xi_{p} \mathbf{J}_{p}^{n-1}\right]\right)
\end{aligned}
$$

where $\nabla \times \mathbf{H}^{n+1 / 2}$ is the standard $\mathrm{H}$ finite differences as listed in equations (3.19)

- (3.21) and:

$$
C_{1 d}=\frac{\frac{1}{2} \sum_{p=1}^{P} \gamma_{p}}{2 \epsilon_{0} \epsilon_{\infty}+\frac{1}{2} \sum_{p=1}^{P} \gamma_{p}+\sigma \Delta t}
$$




$$
\begin{aligned}
C_{2 d} & =\frac{2 \epsilon_{0} \epsilon_{\infty}-\sigma \Delta t}{2 \epsilon_{0} \epsilon_{\infty}+\frac{1}{2} \sum_{p=1}^{P} \gamma_{p}+\sigma \Delta t} \\
C_{3 d} & =\frac{2 \Delta t}{2 \epsilon_{0} \epsilon_{\infty}+\frac{1}{2} \sum_{p=1}^{P} \gamma_{p}+\sigma \Delta t}
\end{aligned}
$$

Note, equation (3.127) reduces to zero for a non-dispersive media. Equation (3.128) reduces to $C_{a}$ for non-dispersive media. Equation (3.129) reduces to $C_{b}$ for non-dispersive media.

To use equation (3.120) and equations )3.126) - (3.129) in the FDTD updating scheme, start with the assumed known (i.e., stored in computer memory) values for $\mathbf{E}^{n-1}, \mathbf{E}^{n}, \mathbf{J}_{p}^{n-1}, \mathbf{J}_{p}^{n}$ and $\mathbf{H}^{n+1 / 2}$ and find the new $\mathbf{E}^{n+1}$ using equation (3.126). Next find the new $\mathbf{J}_{p}^{n+1}$ using equation (3.120) and the newly computed $\mathbf{E}^{n+1}$. Finally, find the updated $\mathbf{H}^{n+3 / 2}$ from the newly computed $\mathbf{E}^{n+1}$ in the usual manner from the Yee algorithm as detailed in equations (3.22) - (3.24), and the updating cycle begins again [34].

To update the grid for the dispersive media using a UPML, the E-update equation given in equation )3.79) must have the update coefficient $C_{4}$ modified from that shown in equation (3.81) to that shown below (notice the removal of the $\epsilon$ in the denominator) [57].

$$
C_{4}^{\prime}=\frac{1}{\left(2 \epsilon_{0} \kappa_{z}+\sigma_{z} \Delta t\right)}
$$


Once equation (3.79) is modified to use the $C_{4}^{\prime}$ update coefficient given in equation (3.130) the output is no longer the electric field intensity $\mathbf{E}$, but still the electric flux density D. To finally calculate the correct $\mathbf{E}$ field use [57]

$$
\left.E_{x}\right|_{i+1 / 2, j, k} ^{n+1}=\frac{\left.D_{x}\right|_{i+1 / 2, j, k} ^{n+1}-\left.\epsilon_{0} \sum_{p=1}^{P} J_{x p}\right|_{i+1 / 2, j, k} ^{n+1}}{\epsilon_{0} \epsilon_{\infty}}
$$

\subsection{Validation of FDTD Calculation}

With the FDTD simulator described in the prior sections, one may ask how accurate is the FDTD method? The accuracy is ultimately dependent on the user. The more cells per wavelength that is selected for the FDTD simulation, the more accuracy the simulator will have. This is especially true for short duration time-domain pulses with a lot of frequency content. Unfortunately, the more cells per wavelength that are selected, the larger the matrix is for the $\mathbf{E}$ and $\mathbf{H}$ fields, thus requiring more memory and more simulation time.

A common technique is to use 40 cells per wavelength at the center of the frequency band and use the data to nominally two times this frequency, since error increases beyond this. Using this type of discretization, the FDTD routine written for this dissertation is tested below. The following test cases are presented:

1. The basic FDTD simulation routine. 
2. Sourcing plane waves into the FDTD grid against a dielectric half-space using the AFP method.

3. Implementation of the UPML.

4. Implementation of the NTFF transform.

5. Implementation of the Lorentz model for dispersive media

\subsubsection{The Basic FDTD Simulation Routine}

The basic FDTD simulation routine is simple to implement in programming languages such as Fortran, $\mathrm{C}$ and MATLAB. A sample of the $\mathrm{TM}_{z}$ polarized wave is presented here (i.e., only $\mathrm{E}_{z}, \mathrm{H}_{x}$ and $\mathrm{H}_{y}$ field components), although the $\mathrm{TE}_{z}$ polarization is just as easy to implement.

The wave is introduced in the FDTD space by means of a simple hard source [34]. A hard source is defined as forcing the E-field (or H-field) to be the same value as the input pulse shape at a specific time. The simulation shown does not include any PML, therefore, the outgoing wave will be reflected back into the FDTD simulation space. For this example, a ricker wavelet centered at $3 \mathrm{THz}$ is used, and the FDTD grid is discretized at 40 cells per wavelength at 3 THz. A ricker wavelet is selected since it is symmetric about the y-axis in the frequency domain and has no DC content. The ricker wavelet is plotted in Figure 3.11. Figure 3.12 shows the ricker wavelet being introduced into the center of 


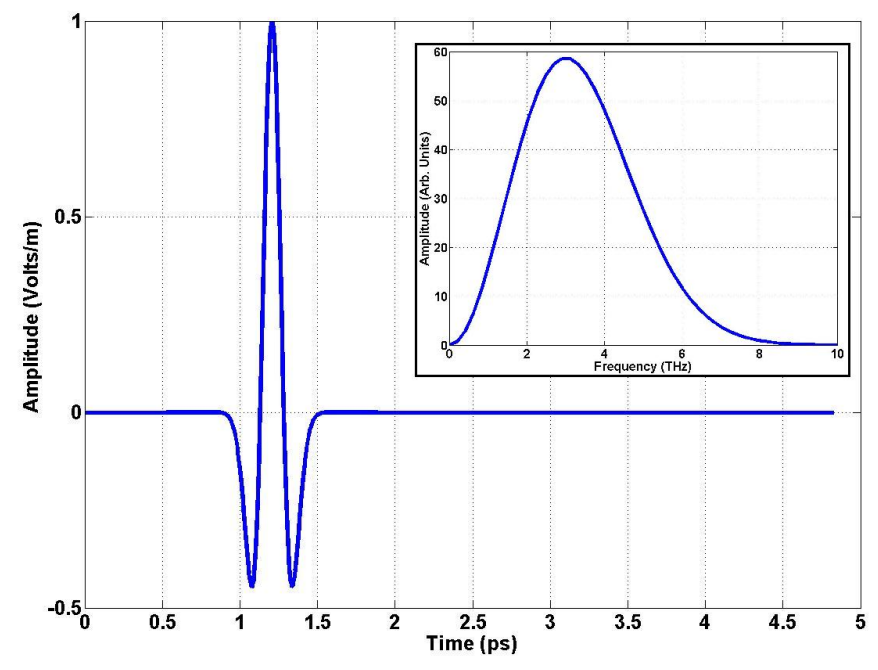

Figure 3.11: Time-domain representation of a ricker wavelet centered at $3 \mathrm{THz}$. Inset is the frequency content of the ricker wavelet.

the FDTD grid by using a hard source, propagating through the FDTD grid, interacting with the edge of the FDTD grid as it reflects off of the edge of the simulation space, and propagating back towards the center after reflecting off of the FDTD grid boundary.

\subsubsection{AFP Implementation of the TFSF Method}

The sourcing of a plane wave is done by using the AFP implementation of the TFSF method as discussed in section 3.2.1. The AFP method is coded and proven in this section for a $\mathrm{TM}_{z}$ polarized wave [39]. This simulation will not have any scatterers in the total-field region and does not include any PML, therefore any wave that leaks into the scattered field region will persist. The source plane wave is a ricker wavelet centered at $3 \mathrm{THz}$. The simulation space is discretized with 40 

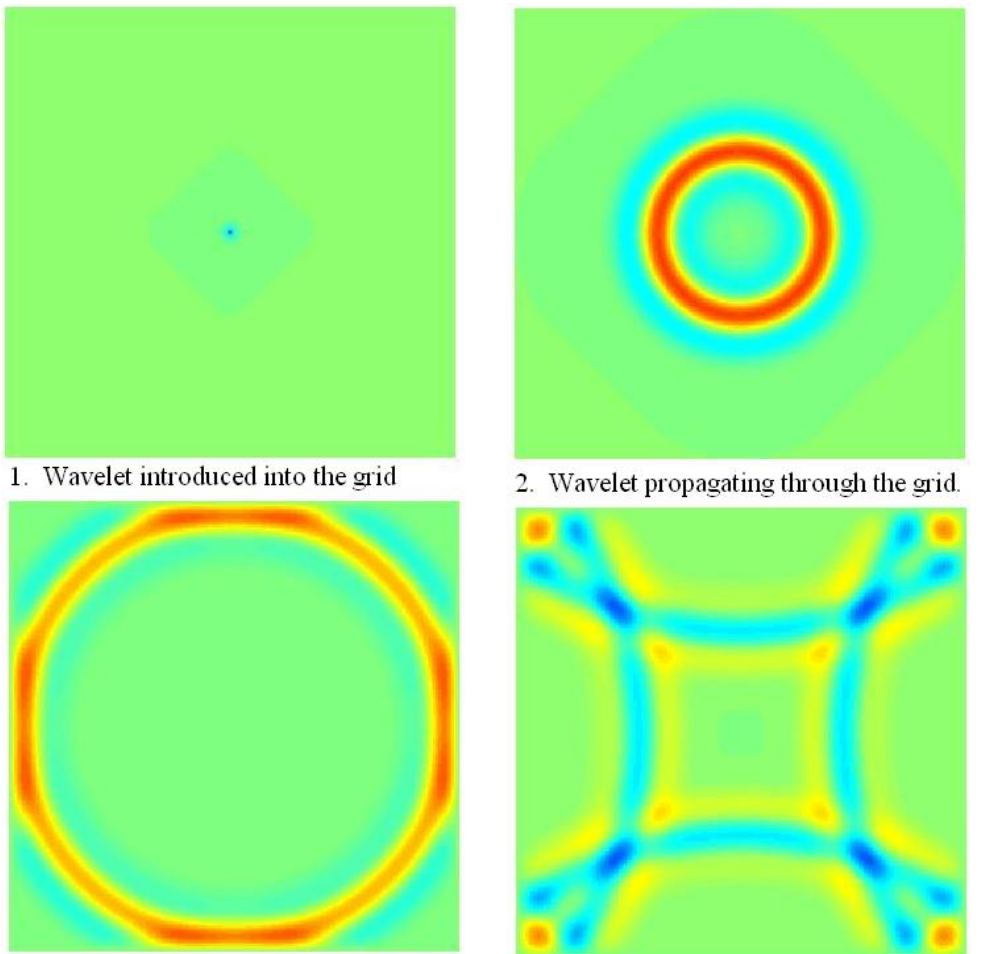

2. Wavelet propagating through the grid.

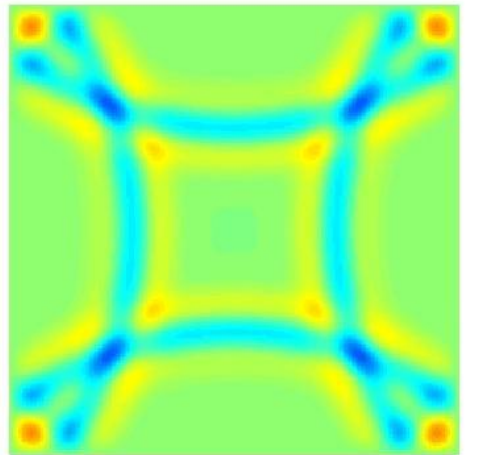

3. Wavelet interacting with the boundary. 4 . Wavelet propagating back to center of grid

Figure 3.12: Ricker wavelet being introduced into, and propagating through, the FDTD grid using a hard source. 
cells per wavelength at $3 \mathrm{THz}$. The bottom half of the plotted simulation space is air $\left(\epsilon_{r}=1\right)$ and the top half is plastic $\left(\epsilon_{r}=4\right)$. The wave is incident at an angle of $60^{\circ}$ measured from the normal.

Figure 3.11 shows the time-domain and frequency-domain response of a ricker wavelet centered at $3 \mathrm{THz}$. Figure 3.13 shows the plane-wave beginning to enter the FDTD grid at the TFSF boundary, the plane-wave interacting with the dielectric interface, and the incident, reflected and transmitted waves in the FDTD grid. Since the AFP method defines the incident and reflected wave, and there as there are no scatterers in the grid, there are no fields in the scattered field region. Also Figure 3.13 shows the plane-wave leaving the FDTD grid at the TFSF boundary. The transmitted wave is moving slower in the plastic, therefore it stays in the FDTD grid longer than the incident wave. In Figure 3.13 the waves are moving from the left side to the right side.

Also shown in Figure 3.13 is an " $\mathrm{X}$ " in the upper right corner. This point was sampled over all time of the FDTD simulation to measure the amount of energy that leaks through the TFSF boundary. Ideally, this number should be zero, however, because of numerical uncertainty, there will be a finite amount of energy that leaks across the boundary. Schneider reported a performance of $1 \times 10^{-10}$ in [40] for an AFP implementation of the TFSF method for free-space only. Figure 3.14 shows leakage less than $1 \times 10^{-9}$, which is of similar performance. The slightly reduced performance is probably due to the calculations for the 


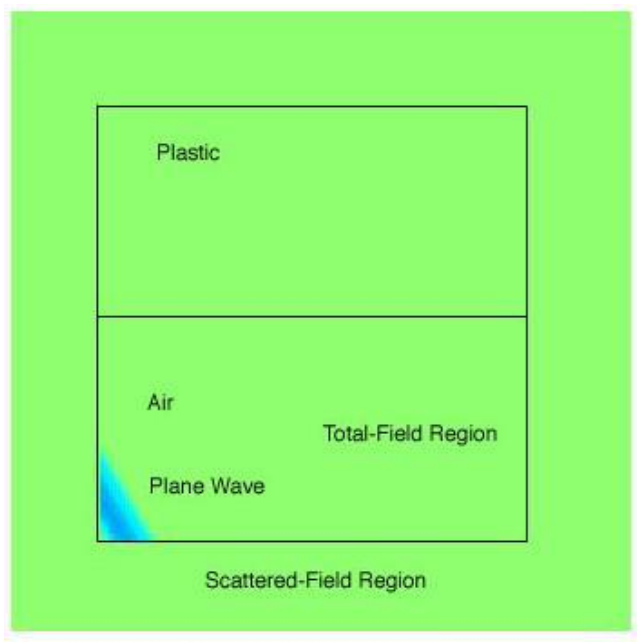

1. Plane-wave entering grid.

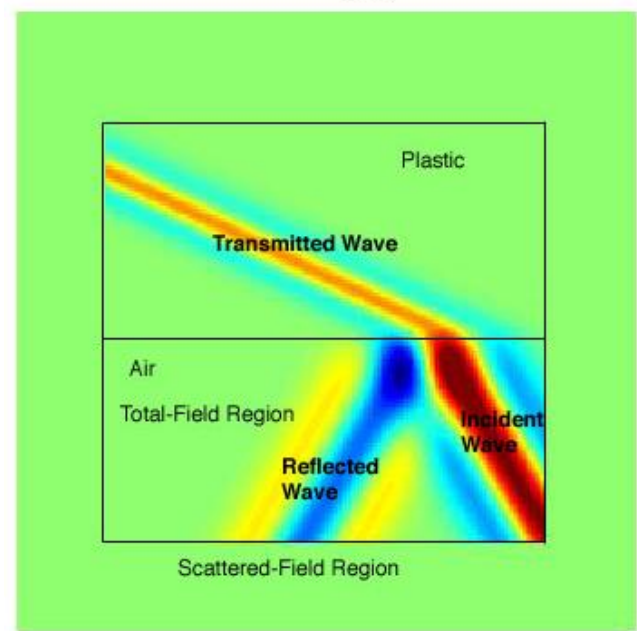

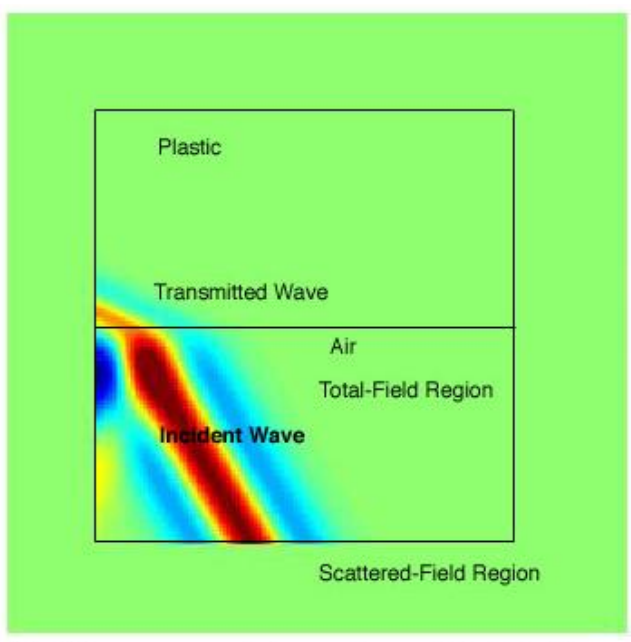

2. Plane-wave interacting with interface.

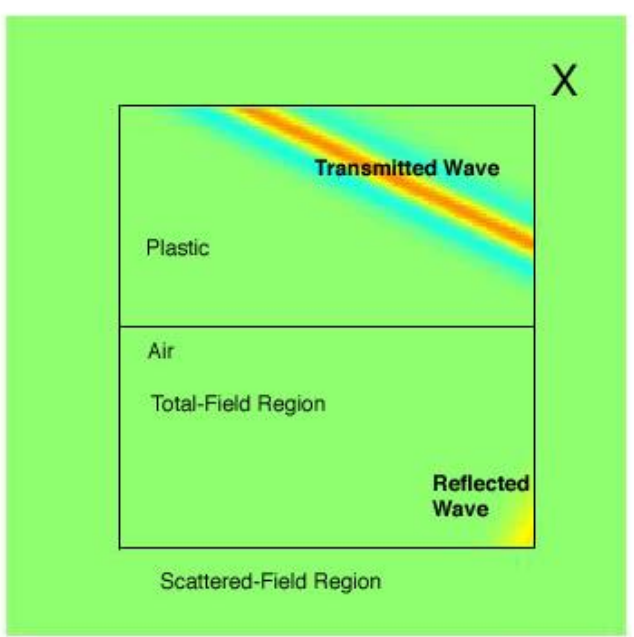

3. Incident, reflected, and transmitted wave visible. 4. Incident wave has left the grid.

Figure 3.13: The plane-wave as it moves through the FDTD grid. The top material is plasitc $\epsilon_{r}=4$ and the bottom material is air $\epsilon_{r}=1$. 


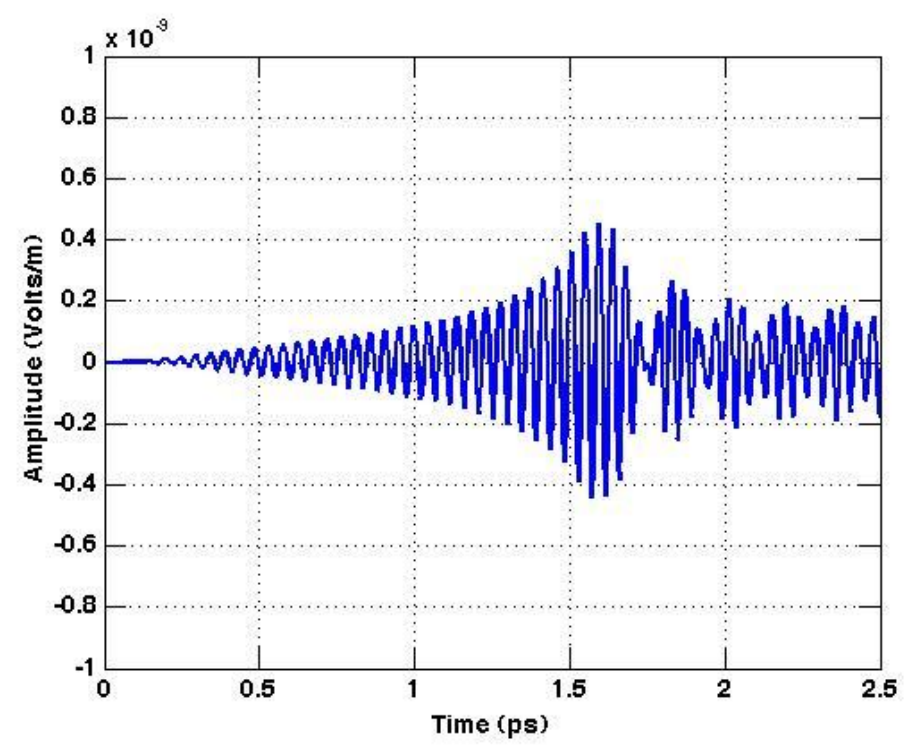

Figure 3.14: Amount of leaked fields at "X" in Figure 3.13

dielectric interface. The fields were measured for enough time that the plane wave would propagate through the FDTD grid and leave the grid at this upper right corner.

\subsubsection{Implementation of UPML}

The PML selected for this work is the UPML. It was selected for the ease of implementation in homogeneous and inhomogeneous materials (including dispersive materials), the reduced computational burden and the improved accuracy when compared to the split field PML. The identical simulation run in the subsection "The Basic FDTD Simulation Routine", which is for a $\mathrm{TM}_{z}$ polarization with a ricker wavelet centered at $3 \mathrm{THz}$ is hard sourced into the FDTD grid discretized at 40 cells per wavelength at $3 \mathrm{THz}$. The UPML is made 10 cells thick to reduce 
spurious reflections.

Figure 3.15 shows the ricker wavelet just entering the FDTD grid, propagating through the FDTD grid, and beginning to interact with the PML layers. Notice that the wave appears to leave the FDTD grid with no reflection. Figure 3.15 shows the ricker wavelet as it almost finishes leaving the FDTD grid. Notice there are no visible reflections. Finally, the ricker wavelet will appear to leave the grid with minimal reflections.

Figure 3.16 shows the magnitude of the electric field at one grid point centered along the UMPL interface. The wave is already beginning to attenuate. While not visible on this scale, there is a small reflection which is $\approx 85 \mathrm{~dB}$ below the incident wave. Figure 3.17 shows the same FDTD cell sampled, but without a UMPL interface. In this cell, the incident wave has not begun to attenuate (as there is no UPML) and there are also multiple reflections shown as the wave reflects from the edge of the FDTD grid.

\subsubsection{Implementation of the NTFF transform}

To test the NTFF transformation, a series of rough metal surfaces were simulated of varying rms heights and correlation lengths as outlined by Tsang [32]. For these simulations, Table 3.2 shows the rough surface statistics used to generate 100 rough surfaces for the Monte-Carlo simulations. Figures $3.18-3.20$ show the results of the FDTD simulation vs. the results published by Tsang [32] for 


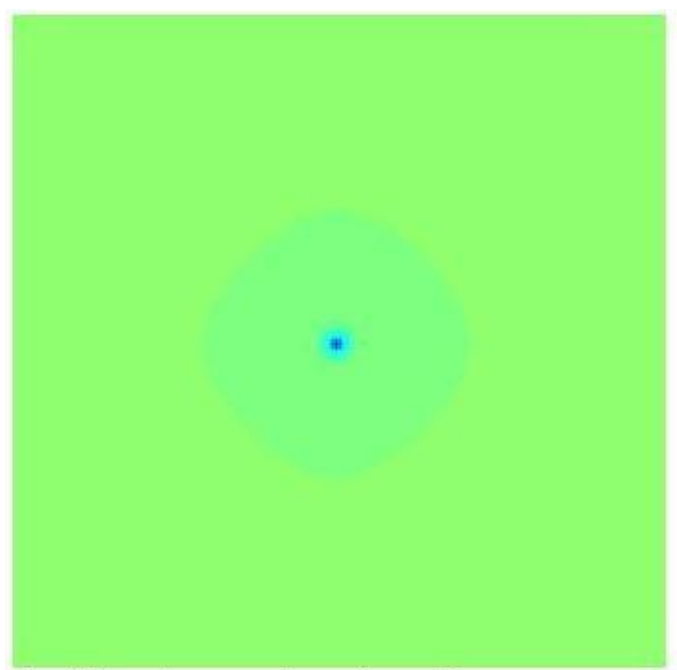

1. Wavelet entering the grid.

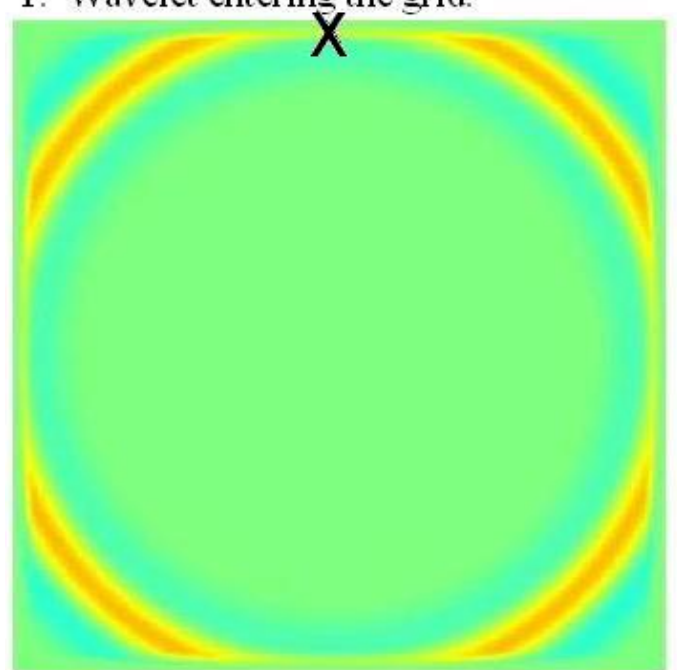

3. Wavelet beginning to leave the grid.

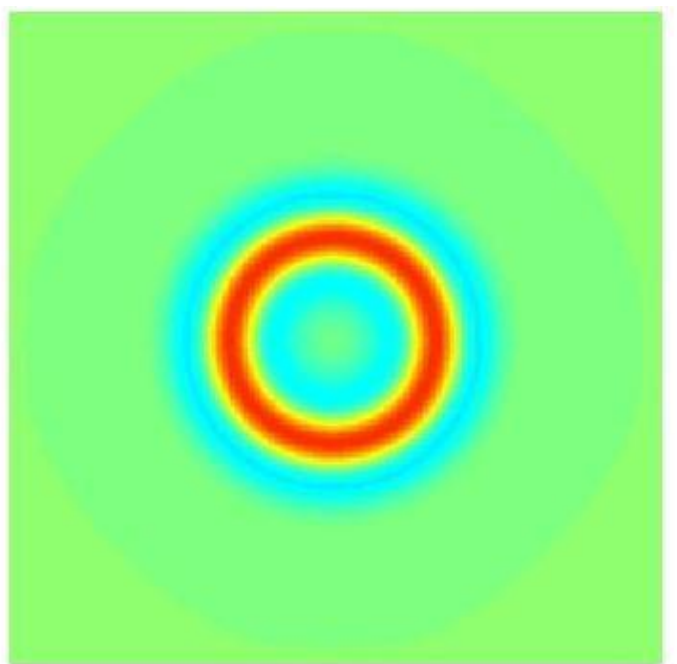

2. Wavelet propagating through the grid

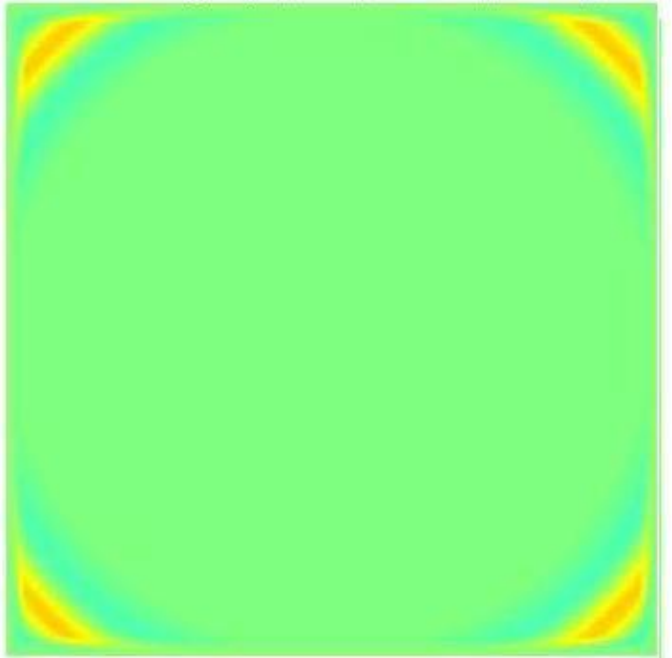

4. Wavelet leaving the grid.

Figure 3.15: Ricker wavelet propagating through the FDTD grid with a UPML. The wave leaves the grid with minimal reflection. 


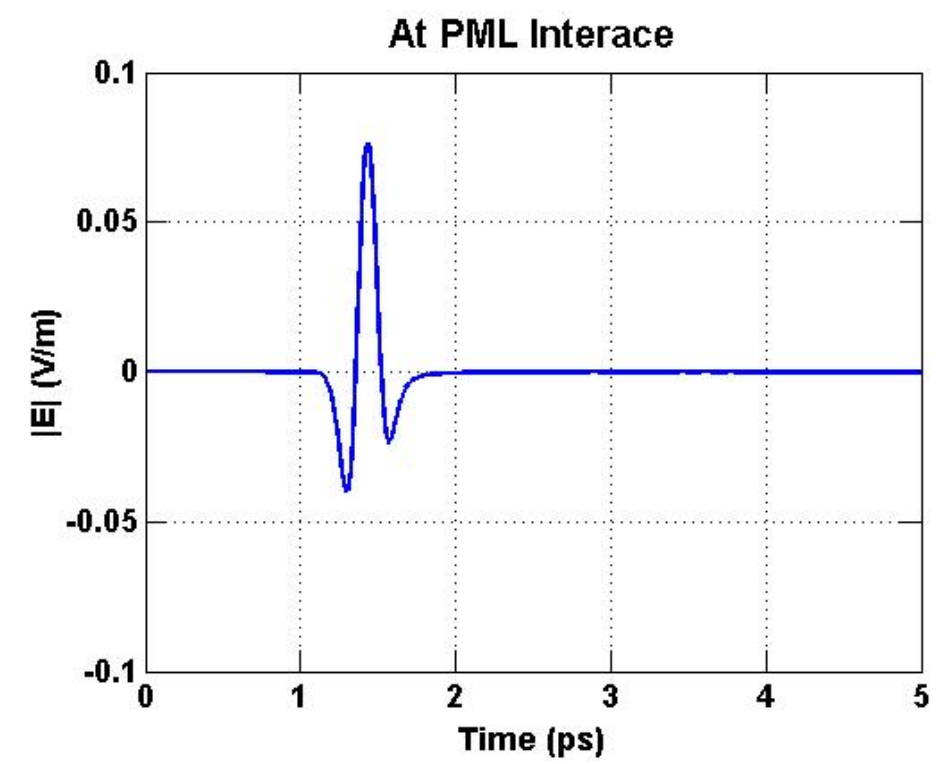

Figure 3.16: E-field over all time of the FDTD simulation sampled at "X" in Figure 3.15 on the PML interface.

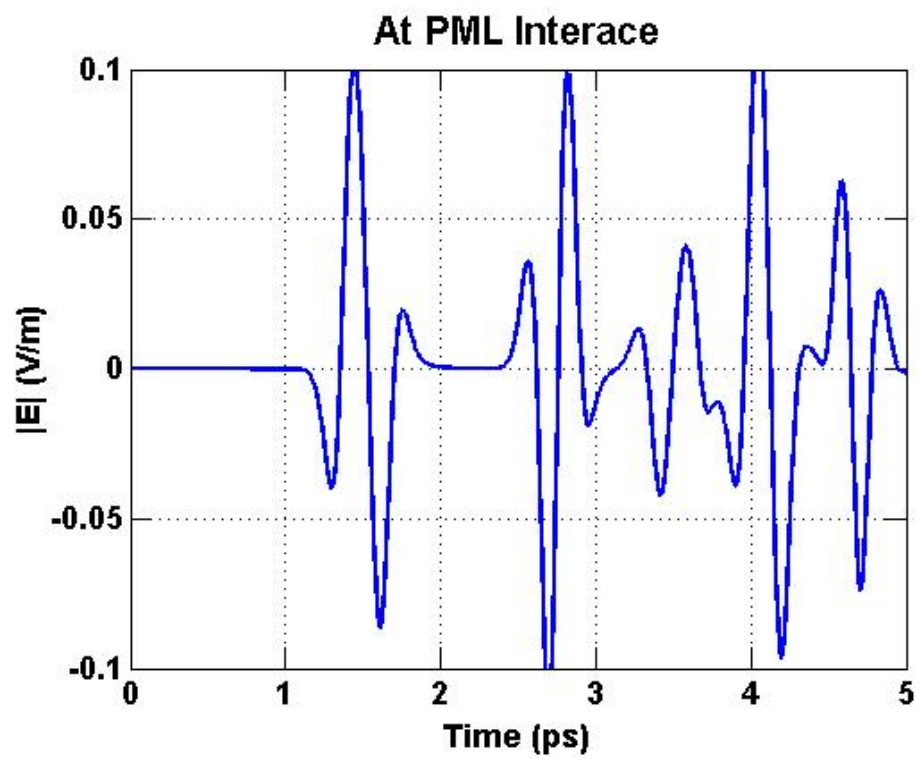

Figure 3.17: E-field over all time of the FDTD simulation sampled at " $\mathrm{X}$ " in Figure 3.15 with no PML interface. 
Table 3.2: Rough Surfaces simulated for NTFF validation. Note, $\lambda$ is for freespace.

\begin{tabular}{|c|c|c|c|}
\hline Simulation & Surface Length & rms height & correlation length \\
\hline 1 & $25.6 \lambda$ & $0.05 \lambda$ & $0.2 \lambda$ \\
\hline 2 & $25.6 \lambda$ & $0.05 \lambda$ & $0.35 \lambda$ \\
\hline 3 & $25.6 \lambda$ & $0.2 \lambda$ & $1.5 \lambda$ \\
\hline
\end{tabular}

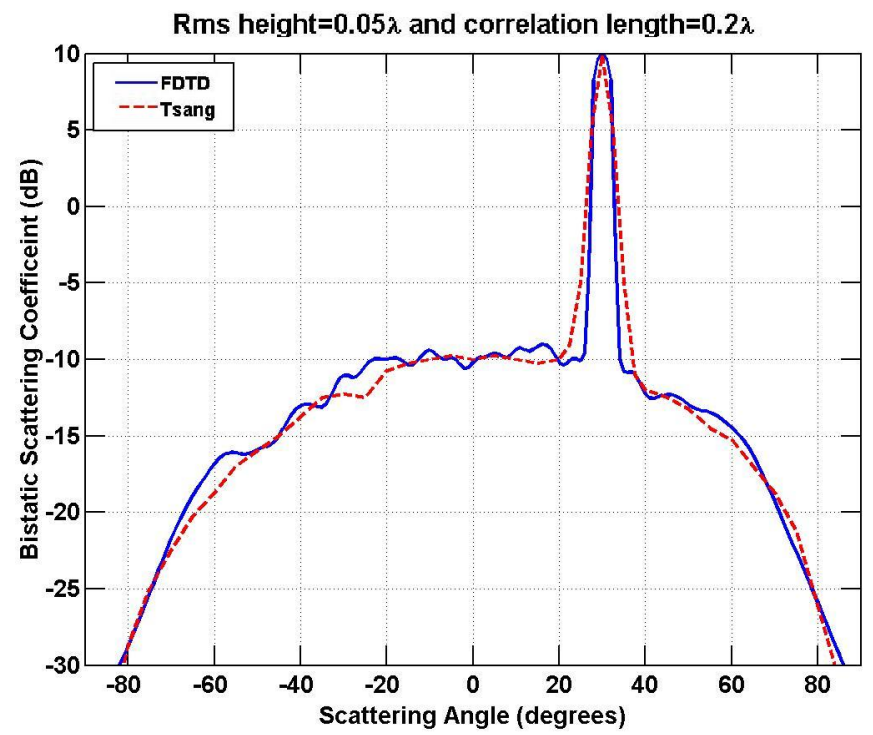

Figure 3.18: Bistatic scattering for a rough metal surface with rms height $=0.05 \lambda$ and a correlation length $=0.2 \lambda . \lambda$ is for free space.

the various simulations. The lengths, rms heights and correlation lengths are given in terms of the free-space wavelength.

\subsubsection{Implementation of the Lorentz model for dispersive media}

To prove the implementation of the dispersive media, the C-4 Lorentz model given in Table 3.1 is entered into the FDTD simulator. The incident plane wave as a ricker wavelet centered at $1.5 \mathrm{THz}$ with the FDTD grid discretized at 40 


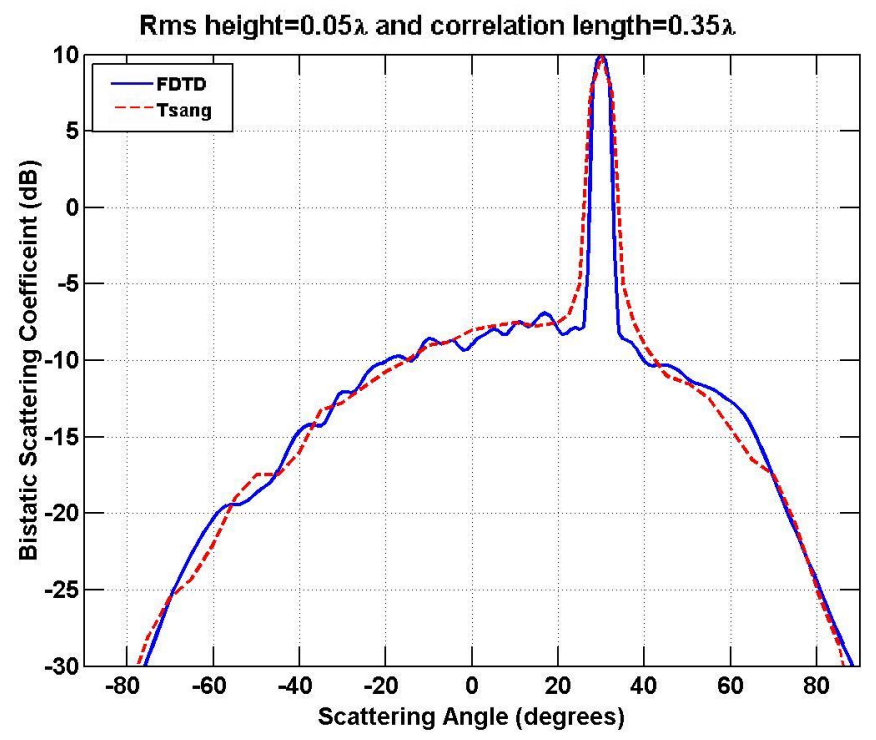

Figure 3.19: Bistatic scattering for a rough metal surface with rms height $=0.05 \lambda$ and a correlation length $=0.35 \lambda . \lambda$ is for free space.

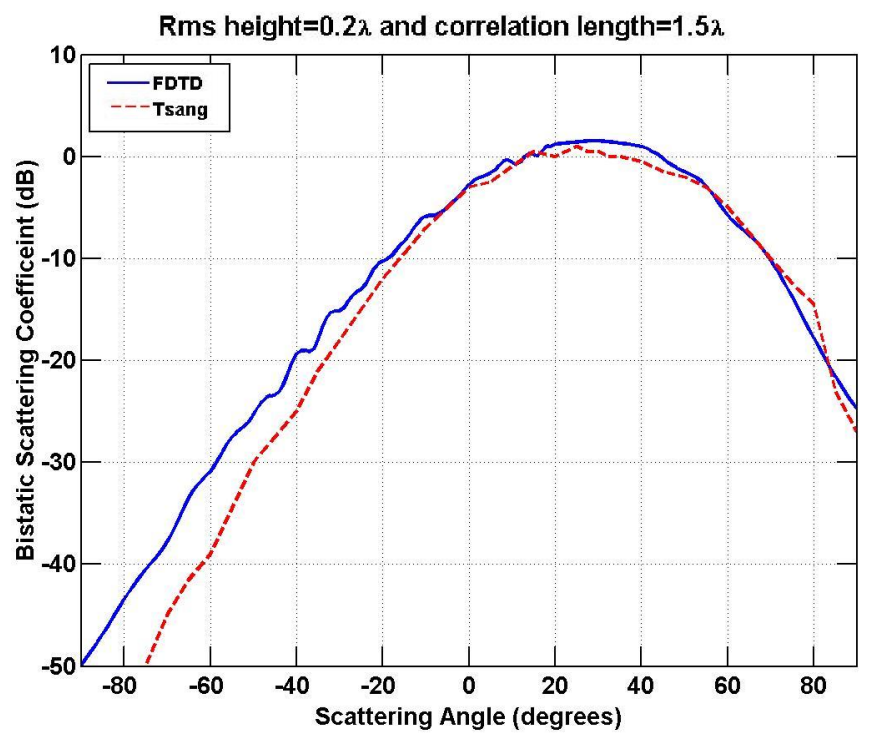

Figure 3.20: Bistatic scattering for a rough metal surface with rms height $=0.2 \lambda$ and a correlation length $=1.5 \lambda . \lambda$ is for free space. 

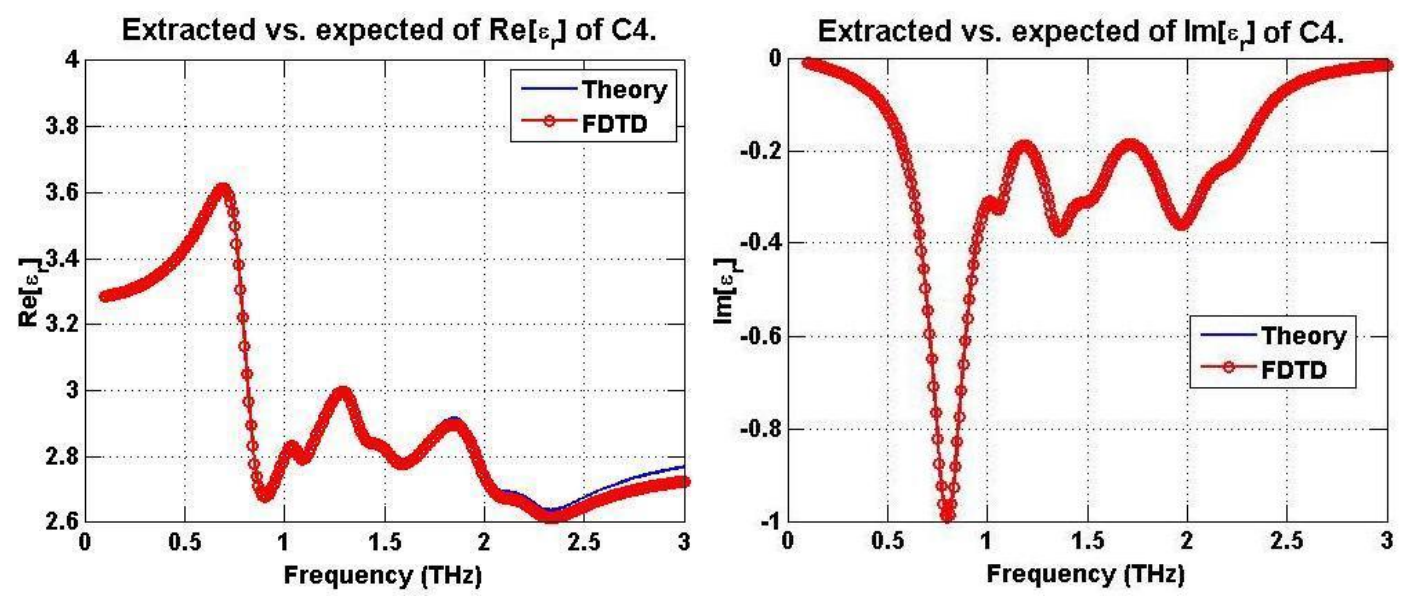

Figure 3.21: The extracted real part of the permittivity vs. the modeled real part of the permittivity.

cells per free-space wavelength at $1.5 \mathrm{THz}$ (e.g. $5 \mu \mathrm{m}$ grid size). The real and imaginary parts of the relative permittivity is then extracted from the FDTD simulation with a plane wave at normal incidence. The results of the modeled C-4 permittivity and the extracted permittivity is given in Figure 3.21.

Figure 3.22 show the percent difference between the modeled permittivity and the extracted permittivity. For increased accuracy, more cells per wavelength could be selected at the cost of increased computational load.

\subsection{Conclusion}

In this chapter the FDTD method was introduced. Several techniques were discussed for the introduction of plane waves, the reduction of spurious reflections, the calculation of far-field responses from the near-field simulations and the implementation of dispersive media. All of these techniques were tested and shown 

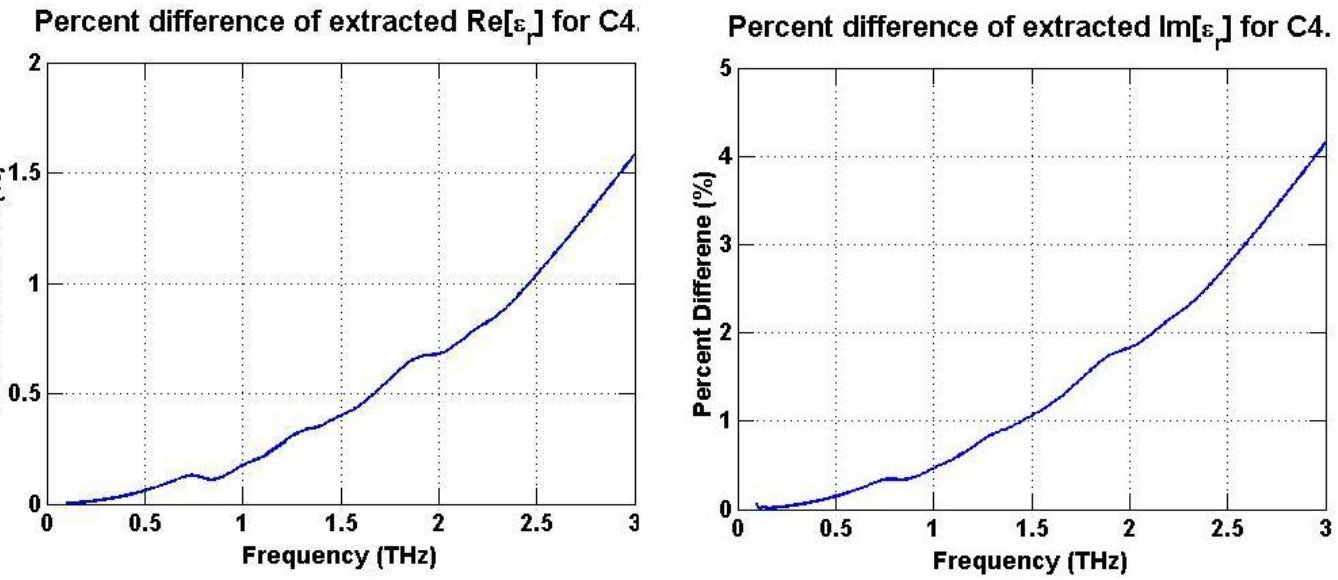

Figure 3.22: Percent difference in the extracted vs. modeled real part of C-4 permittivity.

to be accurate. 


\section{Chapter 4}

\section{Terahertz Measurements}

An FDTD simulator has been created and tested for validity. To gain confidence in its accuracy, a comparison of the proposed modeling methodologies to measured data is needed. This chapter will present data measured with THz measurement systems. It will also compare FDTD model results to the measured data to validate the models used for this work.

\subsection{Terahertz Transmission Measurement and Model Results}

This section explores the granular scattering in a pure sample of compressed polyethylene (PE) powder as measured in the transmission mode in the Laboratory for Nonlinear Optical Studies of Macromolecular Photonic Materials at the University of Maryland, Baltimore Country (UMBC). Two samples were prepared from two lots of photometric grade PE powder. One lot contained PE grains of approximately $20 \mu \mathrm{m}$ in radius (called Small Grain Polyethylene, or SGPE) and the other lot contained grains of approximately $60 \mu \mathrm{m}$ in radius 

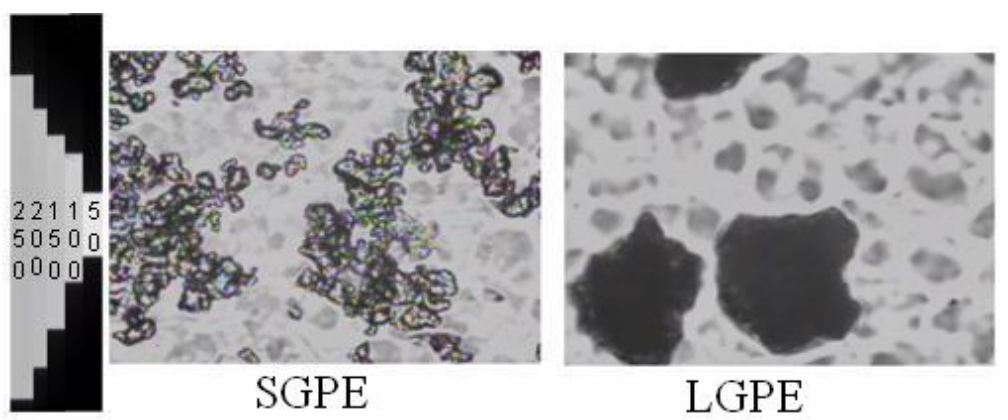

Figure 4.1: Picture of small grain polyethylene (SGPE) on the left and large grain polyethylene (LGPE) on the right from the UMBC THz transmission experiment.

(called Large Grain Polyethylene, or LGPE). Each sample contained 25 to 60 $\mathrm{mg}$ of PE powder compressed into discs of thickness $772 \mu \mathrm{m}$ (for the SGPE) or $1.821 \mathrm{~mm}$ (for the LGPE) using a hand press at approximately 16,000 psi. These results were previously reported using the QCA model in [35]. Pictures of the small grain polyethylene and the large grain polyethylene are shown in Figure 4.1.

The terahertz time-domain spectroscopy of transmission through the PE pellets were conducted at UMBC using the set-up shown in Figure 4.2. This spectrometer performed optical rectification of femtosecond, infrared laser pulses by an electrooptic polymer material to produce short pulses of energy ranging from $0.5 \mathrm{THz}$ to $8 \mathrm{THz}[58,59]$.

To model the two samples with the FDTD method, the simulation space was filled with a slab of PE $\left(\epsilon_{r}=2.1316\right)$ with a thickness of $772 \mu \mathrm{m}$ (for the SGPE) or $1.821 \mathrm{~mm}$ (for the LGPE). To represent the granular nature of the PE pellets, 


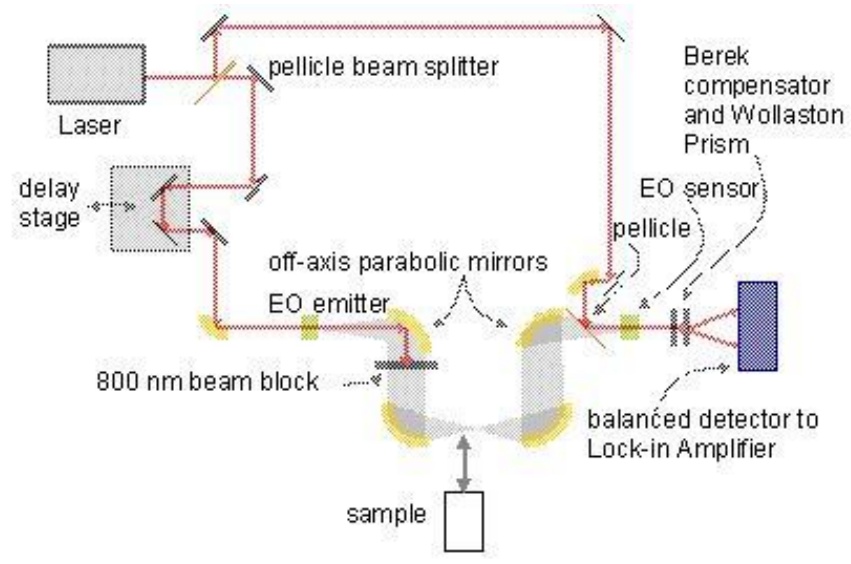

Figure 4.2: Experimental set-up for $\mathrm{THz}$ spectroscopy at UMBC.

the slab was filled with spherical air voids at a fractional volume $\left(f_{v}\right)$ of $20 \%$. A dense packing of the PE grains was assumed, thus equation (4.1) can be used to calculate the size of the spherical air voids [35].

$$
r_{v}=\left[\frac{1}{\sqrt{2} \pi}-\frac{1}{6}\right]^{\frac{1}{3}} r
$$

Utilizing equation (4.1) for the SGPE (with a radius of $20 \mu \mathrm{m}$ ) gives a radius of the air voids as $8 \mu \mathrm{m}$. Utilizing equation (4.1) for the LGPE (with a radius of $60 \mu \mathrm{m})$ gives a radius of the air voids as $24 \mu \mathrm{m}$. Since the air voids will appear in random locations through the slab, a Monte Carlo simulation with 50 samples was run for the SGPE and LGPE samples. For each simulation the scatterers were automatically entered in the FDTD model and care was taken to ensure no scatterers over-lapped, and that sphere centers were not repeated. The number of scatterers was calculated using 


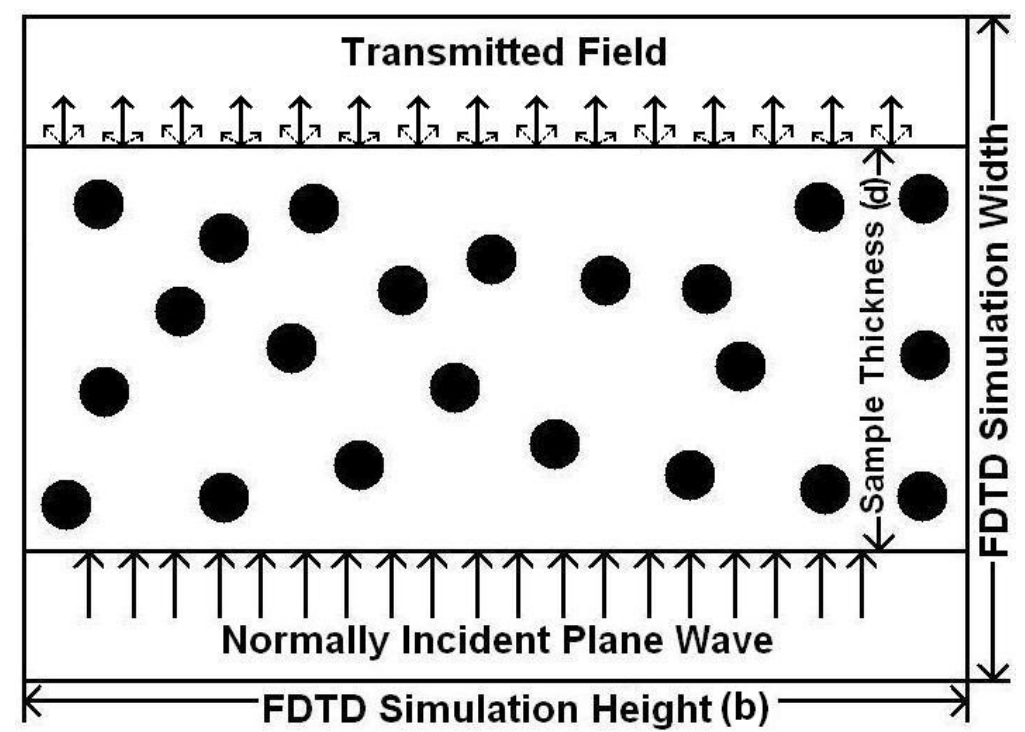

Figure 4.3: FDTD model space for the UMBC scattering simulations. Transmitted field shows coherent (solid line) and incoherent (dashed line) fields.

$$
N_{\text {Scat }}=\frac{d \cdot b \cdot f_{v}}{\pi r_{v}^{2}},
$$

where $N_{\text {Scat }}$ is the number of scatterers, $d$ is the sample thickness, $h$ is the height of the simulation space, $f_{v}$ is the fractional volume of the scatterers, and $r_{v}$ is the radius of the scatterers. The height of the simulation space, $h$, was taken to be 100 times the thickness of the slab, $d$ as shown in Figure 4.3.

The measurement and simulation results were taken at zero degrees (i.e., normal to the surface). Figure 4.4 shows the measured results with the average of the 50 far-field FDTD Monte Carlo simulations. The references curve shown is the spectral response of the UMBC measurement equipment without any sample. The average FDTD simulation results were multiplied by the reference signal to 


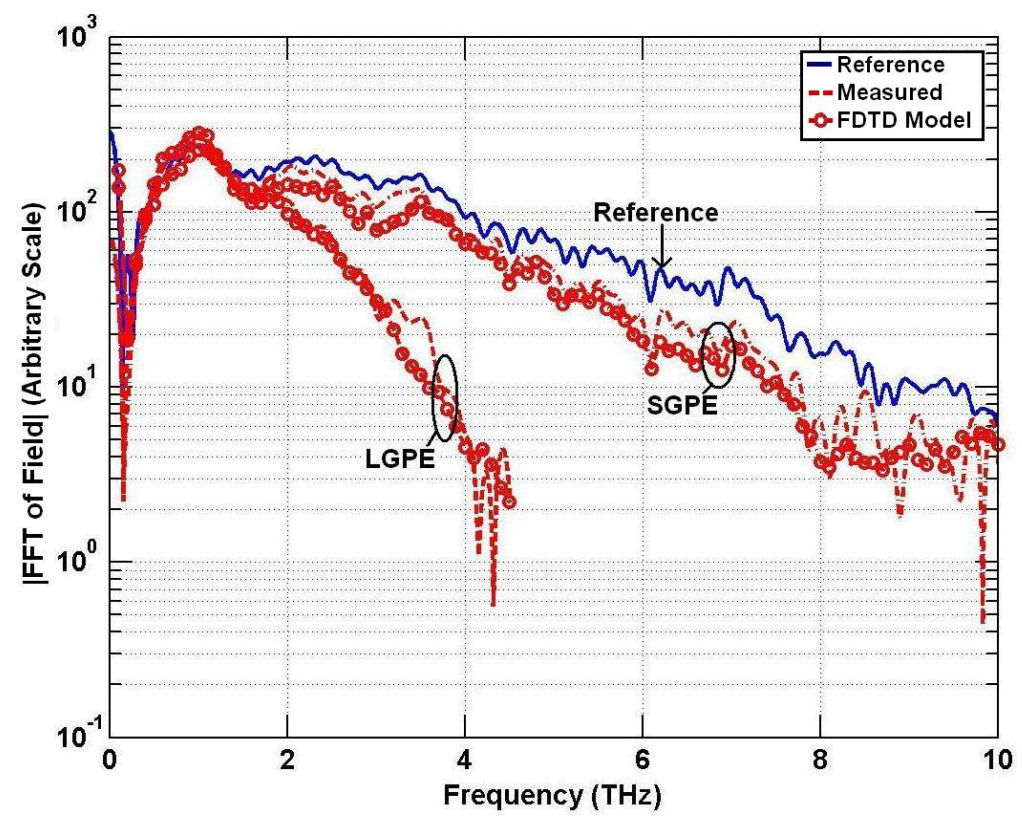

Figure 4.4: Measured (dashed line) vs. modeled (dashed line with circles) response of transmission spectroscopy through PE sample. LGPE data truncated when the measured data reaches the noise floor. The reference curve is shown in blue and the samples are shown in red.

compare the simulations directly to the measured results. While the FDTD curve does not exactly match the measured data, it does predict the different frequency roll-off from the two different mixtures and is within a few $\mathrm{dB}$ of the measured data.

\subsection{Terahertz Reflection Measurements and Model Results}

This section explores rough surface scattering with measurements of terahertz reflection spectra from various grit sandpaper samples which were sputter coated with gold. Sandpaper was selected because it has a controlled surface roughness. 


\section{Gold-coated sandpaper samples}

\section{P80}
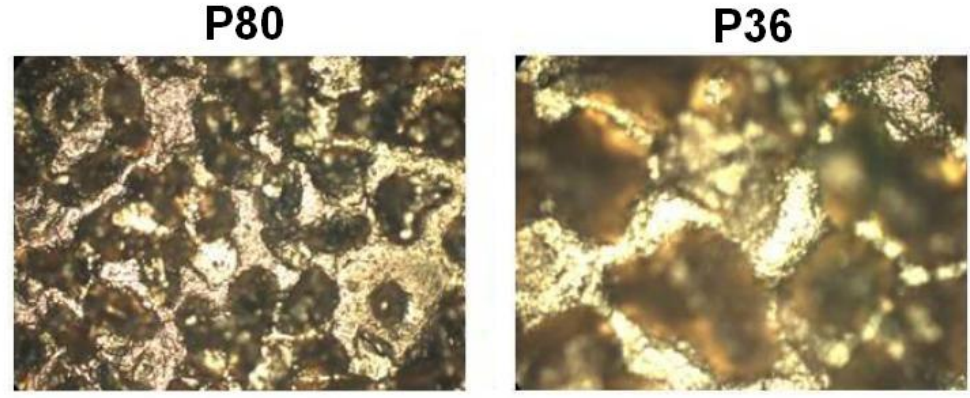

Figure 4.5: Optical picture of gold-coated sandpaper. P80 is the 80 grit sandpaper and P36 is the 36 grit sandpaper.

Table 4.1: Values of RMS height $\left(h_{r m s}\right)$ and correlation length $\left(\mathrm{L}_{C}\right)$ used in the scattering calculations for the sandpaper samples [60].

\begin{tabular}{|c||c|c|}
\hline Grit Value & RMS height, $h_{r m s}(\mu \mathrm{m})$ & Correlation length, $\mathrm{L}_{C}(\mu \mathrm{m})$ \\
\hline 36 & 191 & 315 \\
\hline 80 & 55 & 151 \\
\hline 120 & 21 & 161 \\
\hline
\end{tabular}

The gold coated surface is assumed to be perfectly reflecting, therefore the scattering results are assumed to be due to the rough surface only. Figure 4.5 shows a picture of some of the measured surfaces. The sandpaper surfaces were assumed to be Gaussian with surface statistics for the sandpaper listed in Table 4.1 [60].

The data measured was taken using a Picometrix T-Ray 4000 Time-Domain $\mathrm{THz}$ measurement system at the Northwest Electromagnetics and Acoustics Research Laboratory (NEAR-Lab) at Portland State University (PSU). The T-Ray $4000 \mathrm{THz}$ measurement system was set to have a beam width of $2 \mathrm{~cm}$. The beam 


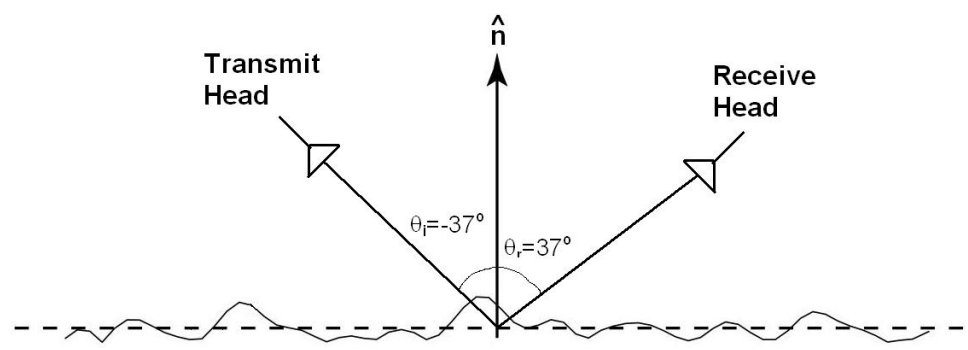

Figure 4.6: Schematic illustration of measurement setup.

was TM polarized, and was weakly cross-polarized ( $20 \mathrm{~dB}$ isolation). No wiregrid polarizers were used to obtain the polarization used for the measurements. The detectors for the $\mathrm{THz}$ system were set to sample the specular scattering from the sandpaper sample, and data was collected using a $100 \mathrm{~Hz}$ scan rate. The angle of the incident $\mathrm{THz}$ beam was approximately $37^{\circ}$ measured from the normal. This is illustrated in Figure 4.6.

The effects of the rough surface on the scattered response is monitored by observing the decrease in the coherent scattered intensity. The 36 and 80 grit sandpaper samples (with roughness profiles listed in Table 4.1) were measured for a single spot with the T-Ray 4000 set to report data after 5 minutes of averaging to increase SNR. This data is then reported in Figure 4.7, which is normalized so the lowest frequency point is at $0 \mathrm{~dB}$. The reported response was normalized by a reference to remove the pulse and instrument spectrum, where the reference was obtained by replacing the sample with a smooth, flat, gold mirror.

To obtain FDTD results, 50 rough surfaces from Table 4.1 were entered into 


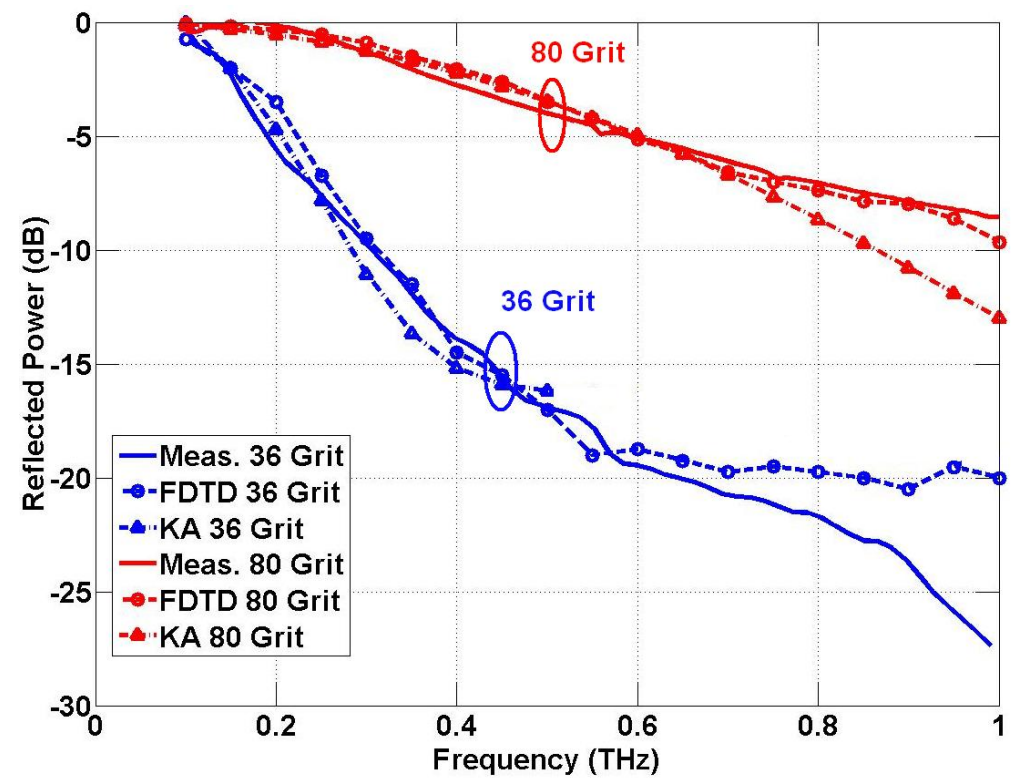

Figure 4.7: Coherent reflected power, relative to a perfectly reflecting surface, for the 36 and 80 grit sandpaper. The measure data is shown without symbols. Monte Carlo FDTD and KA results are lines with symbols.

the FDTD grid for a Monte Carlo analysis. The near-field results were then translated to the far-field. To match with the experimental data, the numerically computed data was normalized by a numerically generated reference as well. The FDTD reference signal was generated by simulating a wave against a perfectly smooth surface (to simulate the reference from the measured data).

Results in Figure 4.7 show the measured data for single spot from the 36 and 80 grit sandpaper with FDTD calculations and predictions from the Kirchhoff Approximation (KA). The FDTD and KA data presented in Figure 4.7 is the mean value (i.e. average) of 50 surfaces. The $\mathrm{KA}$ is only valid when the surface slopes are small compared to a wavelength, and thus are truncated when the 
$\mathrm{KA}$ is no longer valid for the surface. There are differences on the order of 2 $\mathrm{dB}$ between the modeled and measured data up to $0.8 \mathrm{THz}$. Beyond $0.8 \mathrm{THz}$, the variation between the measured 36 grit sample and the FDTD data is explained by the sample to sample variation in the FDTD model. Since the FDTD calculations and KA predictions are for a 1D surface (2D scattering model), the out-of-plane scattering is neglected and this may account for some of the observed difference between the measured and modeled response. It is interesting to note that the measured data is quite close to the mean value, whereas it requires averaging over a number of realizations for the FDTD model. This is due to the additional equivalent averaging inherently present in a 3D measurement (because of the finite width dimension of the sample) relative to a 2D model, and is consistent with results reported previously [61,62].

Figure 4.8 shows the difference in specular return for the three grits of sandpaper listed in Table 4.1 computed using the FDTD model. This figure illustrates the reduced variation from sample to sample as the surfaces become smoother. Therefore, smoother surfaces will require fewer surface realizations to converge to the mean field response.

\subsection{Conclusion}

This chapter presented measured and modeled data of a transmission measurement through a polyethylene sample. It also presented measured and modeled 


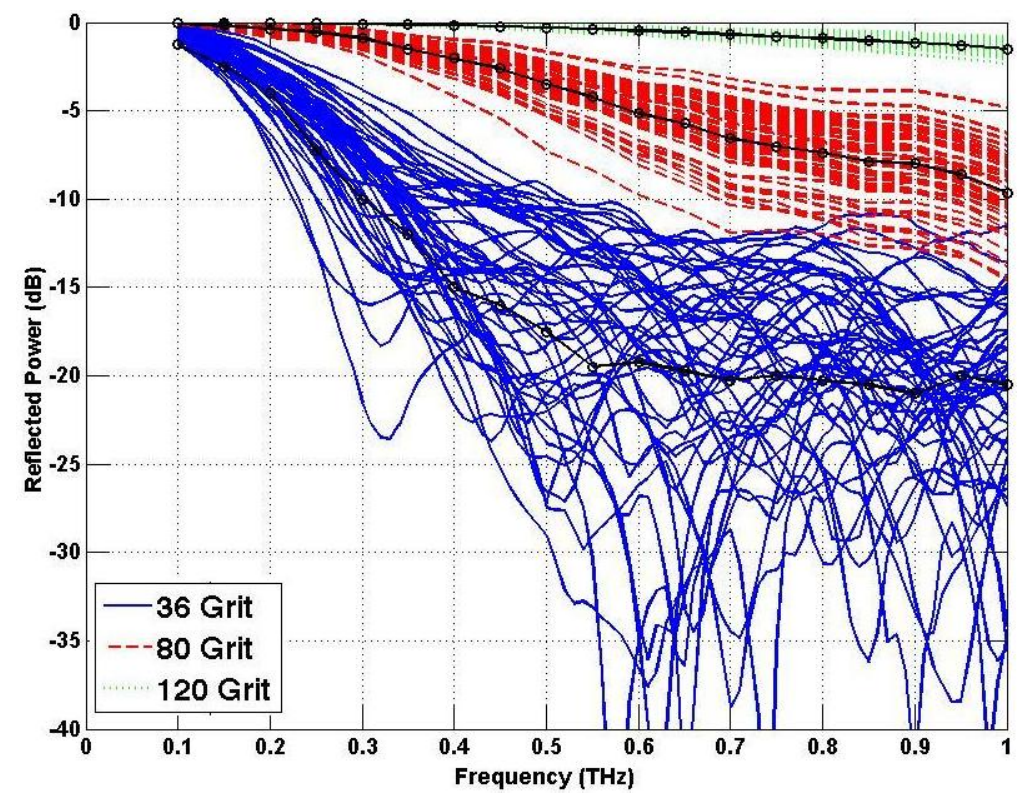

Figure 4.8: Power relative to a perfectly reflecting surface for 36, 80, and 120 grit sandpaper. The mean for each sample is shown in black.

data of rough surface specular reflection. The models were within a few $\mathrm{dB}$ of the measured data, validating the granular scattering and rough surface scattering simulation models. The following chapters will use these validated models to explore the effect of rough surface and granular scattering on the spectral signature of the common explosive Composition-4 (C-4). 


\section{Chapter 5}

\section{Effect of Random Media Interface on Composition-4 Spectral Signature}

The rough surface scattering is expected to play a dominant role in the electromagnetic scattering from the explosives because of the larger dielectric contrast between air the the explosive compared to the dielectric contrast between the filler and the explosive grains. Because of this, the rough surface scattering is studied first. In this chapter, the effect that only the random media interface has on the extracted spectral signature of an explosive material is explored using a FDTD model. The spectral signature of the material will be extracted from the derivative of the reflected power with respect to frequency. However, the reflected power may have some frequency dependence due to the rough surface scattering as well. A filtering methodology will be presented to reduce the contribution of the frequency dependent scattering to allow for the extraction of the material spectral signature. Also, the effect of limited measurement samples on the extracted signature will also be investigated with a lower limit on samples 
needed for reliable detection. The impact of noise and angle of incidence will also be explored.

\subsection{Monitoring Spectral Peaks}

The quantum spectral signature of explosives is modeled as a dispersive material (i.e., a material which the dielectric constant varies with frequency). To determine the location of the poles in the Lorentz model (which are used to model the absorption and dispersion signature of the material), the derivative of the reflected power will be employed [63]. The Lorentz parameters from Table 3.1 are entered into the FDTD simulator, and a plane wave (at normal incidence) strikes the surface of the dispersive explosive Composition-4 (C-4) which is made predominately of RDX. The amount of reflected power is shown in Figure 5.1 and the derivative of the reflected power is presented in Figure 5.2. Also presented in these figures is the theoretical calculation from the Fresnel reflection coefficients. The purpose of this is to show that the derivative of the reflected power will show the correct location of the Lorentz poles. The location of these poles will be used to study the impact a rough surface may have on the spectral signature of the explosive C-4. As stated in Chapter 3, the Lorentz poles found from taking the derivative of the reflected power will be at a slightly higher frequency than the absorption peaks. 


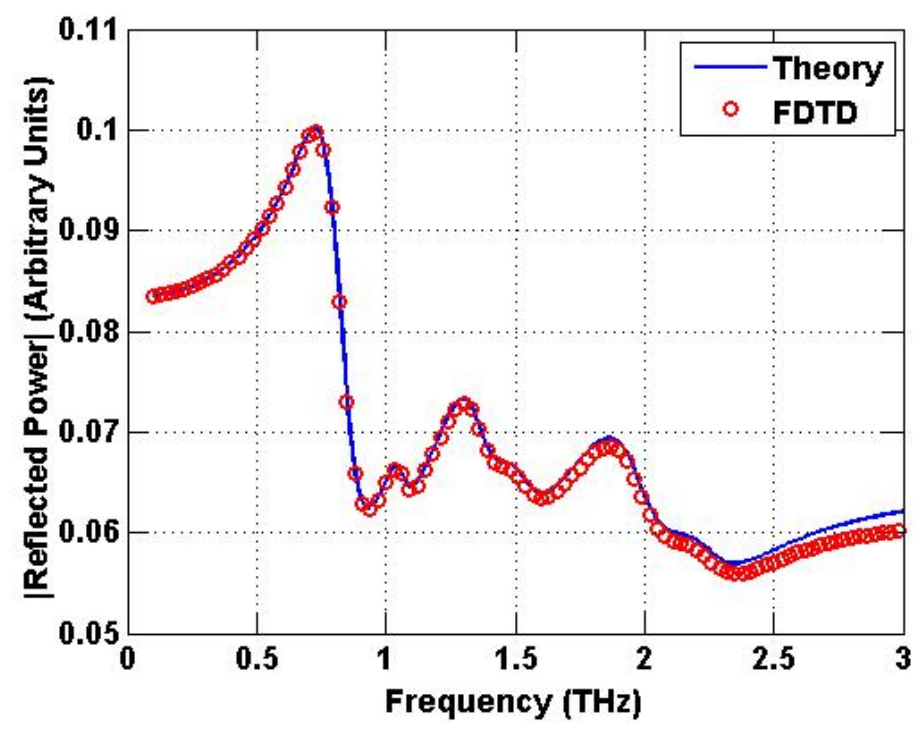

Figure 5.1: Comparison of the reflected power between FDTD results and calculated results for a wave of normal incidence.

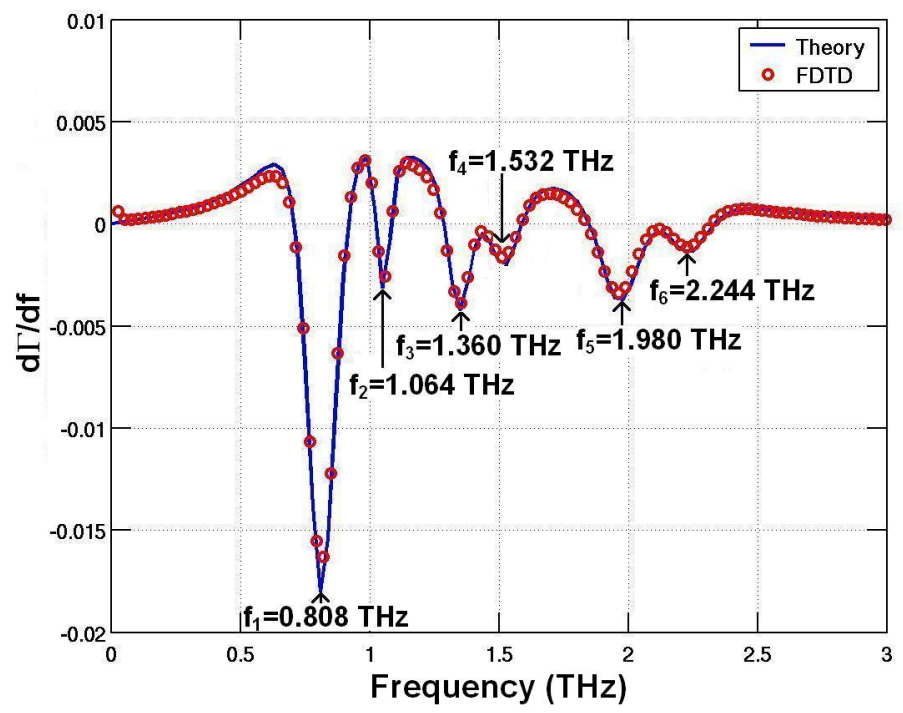

Figure 5.2: Comparison of the derivative of the reflected power between FDTD results and calculated results for a wave of normal incidence. The location of the peaks is the same as that listed in Table 3.1. 


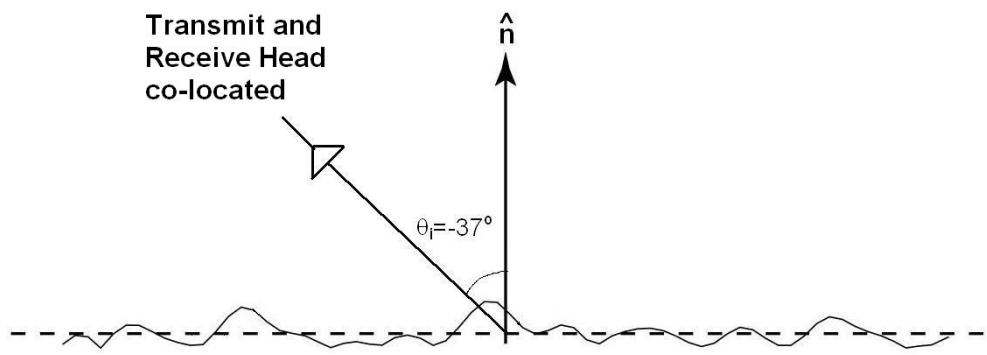

Figure 5.3: Schematic representation of rough surface simulations.

\subsection{Impact of Rough Surface on Composition-4 Spectral Signature}

To study the effect of a rough surface on the spectral signature of the C-4, a FDTD simulation is computed assuming a rough surface for the C-4 material interface with statistics of either 36 grit or 80 grit sandpaper (as reported in Table 5.1) [60]. The angle of incidence (measured from the normal) is $-37^{\circ}$ for both roughness profiles. Fifty different $2 \mathrm{~cm}$ long surfaces (discretized at 40 cells per wavelength at $1.5 \mathrm{THz}$ ) for each simulation case is run for the Monte Carlo analysis. A monostatic (i.e., transmitter and receiver are co-located resulting in a detection angle of $-37^{\circ}$ ) detection regime is assumed for this simulation to more closely match a possible real-world application where it is often not possible to place a receiver in the specular direction to maximize received power. This is illustrated in Figure 5.3.

Figure 5.4 shows an example of the FDTD simulated RCS (also called the Bistatic Scattering Coefficient) of C-4 with a surface roughness of 36 and 80 grit sandpaper at $0.5 \mathrm{THz}$. The specular peak has totally disappeared for the 36 
Table 5.1: Values of RMS height $\left(h_{r m s}\right)$ and correlation length $\left(\mathrm{L}_{C}\right)$ used in the scattering calculations for the sandpaper samples [60].

\begin{tabular}{|c||c|c|}
\hline Grit Value & RMS height, $h_{r m s}(\mu \mathrm{m})$ & Correlation length, $\mathrm{L}_{C}(\mu \mathrm{m})$ \\
\hline 36 & 191 & 315 \\
\hline 80 & 55 & 151 \\
\hline
\end{tabular}

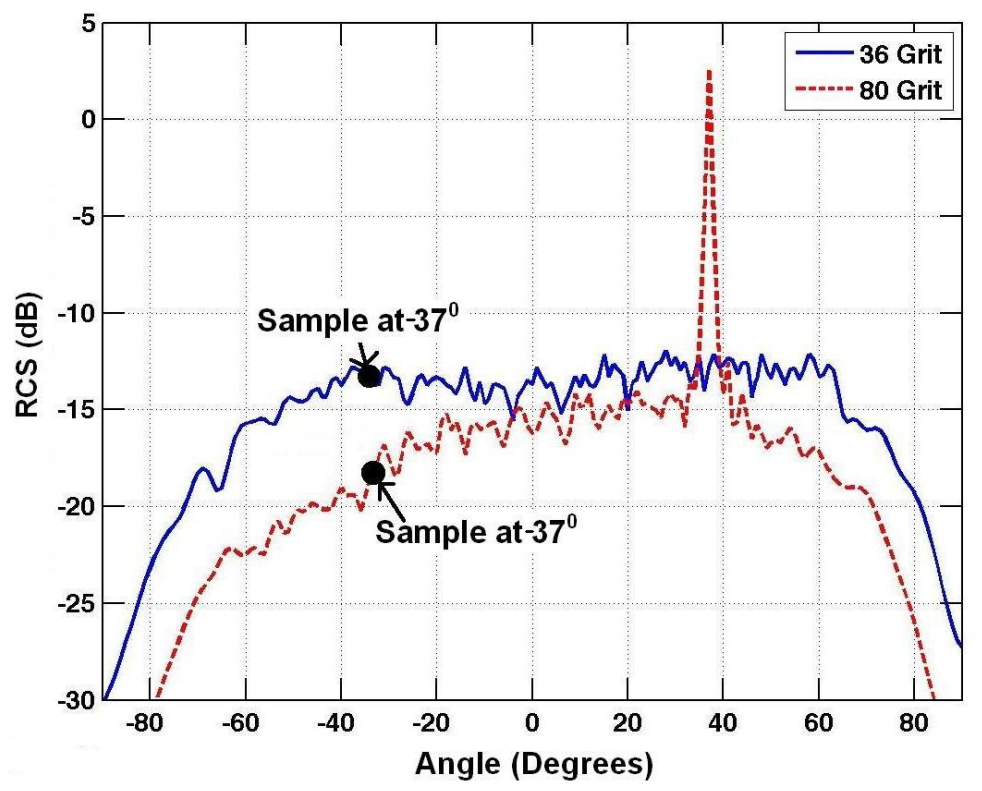

Figure 5.4: Representative RCS at $0.5 \mathrm{THz}$ of homogeneous $\mathrm{C}-4$ with a surface roughness of 36 and 80 grit sandpaper. Angle of incident plane wave is $-37^{\circ}$ measured from the surface normal. Fifty surfaces are averaged.

grit surface because the incoherent scattering has a larger magnitude than the coherent scattering.

The FDTD simulations include far-field data at all receive angles and frequencies. The FDTD RCS data is averaged over a $5^{\circ}$ window centered at a receive angle of $-37^{\circ}$ (relative the normal as illustrated in Figure 5.4) to approximate a receiving antenna with a finite width main lobe. The magnitude of the RCS data 


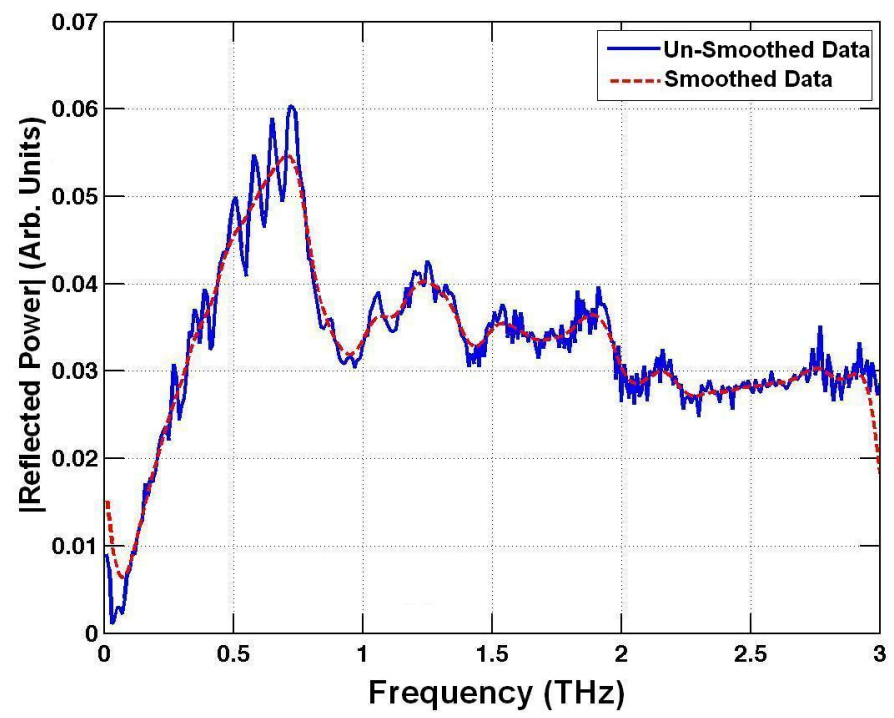

Figure 5.5: FDTD simulated RCS data of homogeneous C-4 sampled over frequency in the backscatter direction for 36 grit sandpaper. Fifty surfaces are averaged.

vs. frequency for the 36 grit surface as averaged in the window centered at $-37^{\circ}$ is shown in Figure 5.5.

Figure 5.4 and 5.5 show the RCS data sampled around $-37^{\circ}$ is not a smooth curve due to the rough surface scattering. The variability of the sampled data will make it difficult to extract the Lorentz poles of the C-4 by taking the derivative of the spectral data as the slope is continuously changing. However, comparing the rough surface reflected power data in Figure 5.5 to the ideal reflected power data of Figure 5.1 shows a very similar shape. So the spectral peaks are in the data, but a new way to extract the spectral peaks is required.

To use the derivative method to extract the Lorentz poles, the data must be smooth. If the un-smoothed data in Figure 5.5 were a time-domain signal, then 
the roughness can be considered as a small-period (fast varying, high frequency) signal superimposed on the actual signal. To remove this small-period data, a low-pass filter can be applied to leave only the slowly varying signal. To filter the data take the Fast Fourier Transform (FFT) of the frequency sampled data as shown in Figure 5.5, multiply the FFT domain data by a gaussian shaped filter (with a standard deviation of $1 / 12^{\text {th }}$ the sample space), and take the inverse FFT of this product. This is similar to acoustic analysis performed in the cepstrum dominan [64]. Mathematically, this is written as

$$
S(f)=\Im^{-1}\left(\Im[R(f)] \cdot G_{f}\right)
$$

where $\Im$ is for the FFT, $\Im^{-1}$ is for the inverse FFT, $S(f)$ is the smoothed signal in the frequency domain, $R(f)$ is the sampled RCS data from the FDTD simulator in the frequency domain, and $G$ is the Gaussian shaped filter in the FFT domain used to smooth the FDTD RCS data. The result is the smoother curve labeled "Smoothed Data" in Figure 5.5. The derivative of the smoothed data for the two roughness profiles are shown in Figure 5.6. The location of the Lorentz poles is again presented in Table 5.2.

The threshold for detection of the peaks will generally be set to be within $5 \%$ of the expected Lorentz pole locations. This is $5 \%$ threshold is selected by looking at the frequencies listed in Table 2.3. For the base explosive materials 
Table 5.2: Lorentz poles with a \pm 5\% range for C-4 [10].

\begin{tabular}{|c|c|c|c|}
\hline Pole & $f_{p}(\mathrm{THz})-5 \%$ & $f_{p}(\mathrm{THz})$ & $f_{p}(\mathrm{THz})+5 \%$ \\
\hline 1 & 0.76 & 0.808 & 0.85 \\
\hline 2 & 1.01 & 1.064 & 1.12 \\
\hline 3 & 1.29 & 1.356 & 1.42 \\
\hline 4 & 1.45 & 1.532 & 1.61 \\
\hline 5 & 1.88 & 1.980 & 2.08 \\
\hline 6 & 2.13 & 2.244 & 2.36 \\
\hline
\end{tabular}

of PETN, RDX, TNT, and HMX, most of the frequencies are farther apart than 5\%. RDX and PETN have a single common frequency, but the presence of other poles will allow for differentiation. HMX also has a frequency near RDX and PETN which may confuse detection, however this is the lowest frequency pole location for HMX, so the other higher frequency poles of HMX will also allow for discrimination of HMX as well.

Based on the $5 \%$ detection threshold for the peaks found from the derivative of the reflected power, the following algorithm will be used to find the peaks.:

1. "Null" must be below zero.

2. Look for the minimum (i.e., the deepest part of the null) within $5 \%$ of each of the frequencies listed in Table 5.2

3. If the frequency is not found within $5 \%$, move the range to $10 \%$ and look again. While $10 \%$ may allow for confusion between explosives, the location of other lower and higher frequency peaks may still allow for positive 


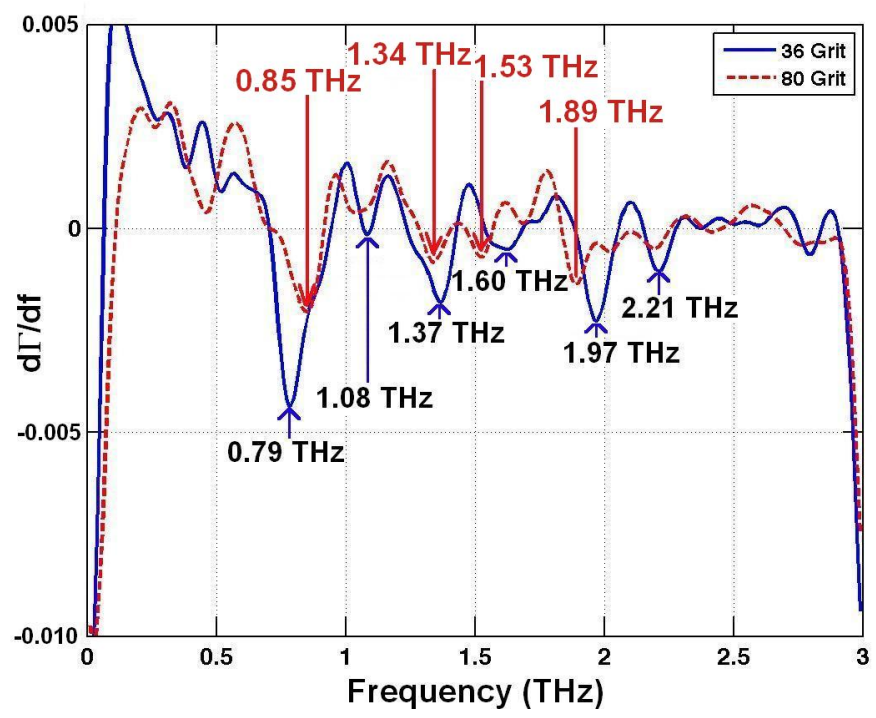

Figure 5.6: Derivative of smoothed data sampled from the FDTD RCS data sampled in the backscatter direction from a homogeneous C-4 sample with the surface roughness listed in Table 5.1. Fifty samples were averaged. The arrows correspond to the location (within 5\%) of the Lorentz poles from Table 5.2.

identification.

4. Report the location of all of the frequency nulls found.

For the 36 grit roughness, the Lorentz poles as listed in Table 5.2 at approximately $0.808,1.064,1.360,1.532,1.980$, and $2.244 \mathrm{THz}$ are located within $5 \%$ of the smooth surface values. For the 80 grit roughness profile, only the peaks near $0.808,1.360,1.532,1.980$ and $2.244 \mathrm{THz}$ are visible and within $5 \%$. The peak near $1.064 \mathrm{THz}$ is seen, but not deep enough for detection. Also, the 80 grit surface has more false peaks. Since the the 80 grit surface has less non-specular energy, there is more frequency dependent scattering in the backscattered direction due to the surface than the 36 grit surface. If the RCS curve for the 80 
grit surface was plotted for all frequencies, the shoulder of the RCS curve will be coming up as the frequency increases until the specular peak disappears. Since the specular peak of the 36 grit surface is lost at a lower frequency than the 80 grit surface, the 80 grit surface will show more frequency dependence in the backscattered response until the specular peak is lost. The increased frequency dependent scattering from the surface roughness may lead to the false peaks.

Figure 5.6 shows the location of most of the Lorentz poles of $\mathrm{C} 4$ listed in Table 5.2 within $\pm 5 \%$. However, there are many false peaks in the data. These false peaks can be filtered out if the width of the Gaussian filter used in Equation (5.1) is swept from $1 / 12^{\text {th }}$ of the sample space to $1 / 24^{\text {th }}$ of the sample space (i.e., from a wider filter to a narrow filter). However, for the 36 grit case, more filtering suppresses the peak near $1.064 \mathrm{THz}$ as shown in Figure 5.7. More filtering also reduces the depth of the real peaks.

Also, as the number of surfaces included in the averaging is increased, the number of false peaks is decreased. Therefore, less filtering will be needed with more surfaces available.

\subsection{Impact of Surface Samples on Composition-4 Spectral Signature}

The prior section assumed there were a sufficient number of realization (50) to adequately estimate the mean field response of a one-dimensional surface. In a 


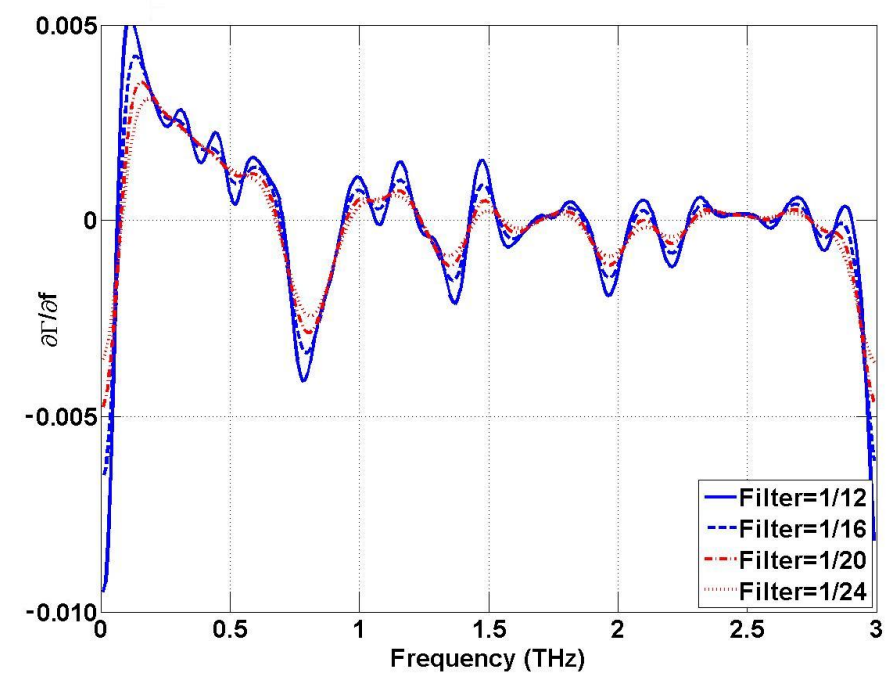

Figure 5.7: Derivative of smoothed data sampled from the FDTD RCS data sampled in the backscatter direction from a homogeneous C-4 sample with a 36 grit surface roughness listed in Table 5.1. Fifty samples were averaged. Width of the Gaussian filter is swept from $1 / 12^{\text {th }}$ to $1 / 24^{\text {th }}$ of the sample space.

practical measurement application with a finite beam width, it may not be possible to measure enough unique locations of a sample to realize the mean response. In addition, a measurement system will be a full three-dimensional system (i.e. a two-dimensional rough surface). With a two-dimensional rough surface, fewer realizations are needed to realize the mean field [65]. So a measurement system will need fewer measurements than a one-dimensional simulation to realize the mean field. Regardless, if insufficient surface realizations are available to realize the mean field, some of the peaks may become obscured. For example, if only 10 surfaces are averaged, then the derivative of the sampled data (after smoothing with a filter width of $1 / 12^{t h}$ the space using equation (5.1)) is shown in 


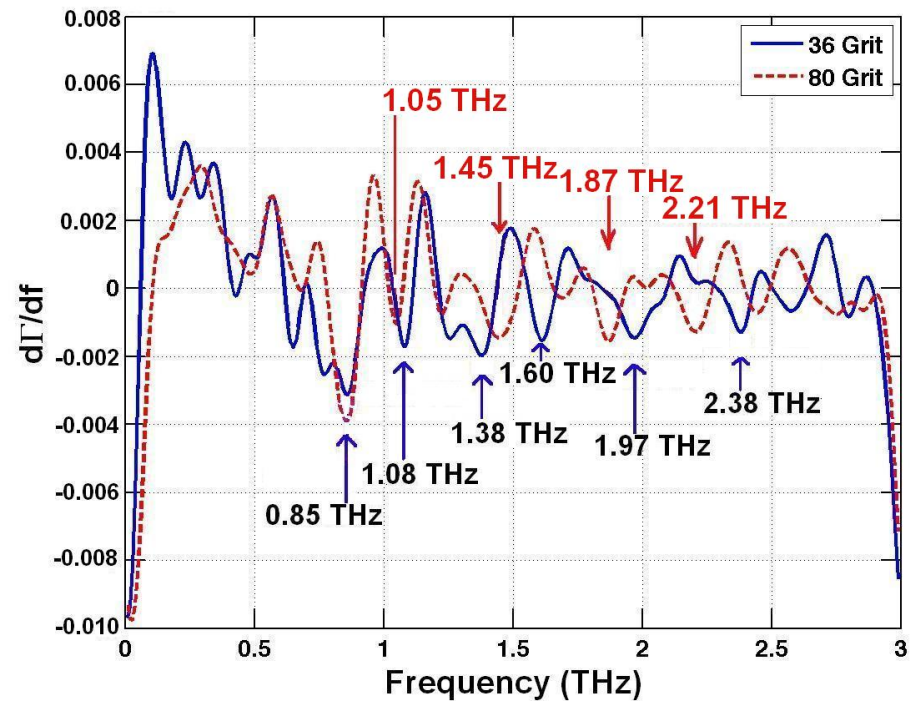

Figure 5.8: Derivative of smoothed data sampled from the FDTD RCS data sampled in the backscatter direction from a homogeneous C-4 sample with the surface roughness listed in Table 5.1. Ten samples were averaged. The arrows correspond to the location (within $5 \%$ ) of the Lorentz poles from Table 5.2.

Figure 5.8.

For the 36 grit surface, the peaks near $0.808,1.064,1.360,1.532$, and 1.980 are with $5 \%$ of the expected location. Only the $2.244 \mathrm{THz}$ peak has moved more than $5 \%$, but is within $10 \%$ of the expected value. For the 80 grit surface, the $0.808,1.064$, and $2.244 \mathrm{THz}$ peaks are within $5 \%$ of the expected value. The other three peaks near $1.360,1.532$, and $1.980 \mathrm{THz}$ are visible, and within $10 \%$ of the expected values.

It is worth noticing that the detected peaks are surrounded by false peaks, which may impair the reliability of identifying the explosive. The additional false peaks are caused by the increased variability in the reflected power since there 
are not enough surface realizations to adequately estimate the mean field. In practice, since the surfaces will be random, the location of the false peaks will likely be random in location, and may hamper detection. However, the false peaks can be reduced, or eliminated, by increased filtering at the cost of losing some of the actual peaks.

\subsection{Impact of Multiple Surface Samples on Composition-4 Spectral Signature}

In the prior section, 50 simulation cases were run for the Monte-Carlo analysis for the investigation. When all 50 surfaces were averaged, the Lorentz poles were visible, and within $5 \%$ of the expected location for both surfaces. When the number of surfaces averages was reduced to 10, it was still possible to see many of the peaks within $5 \%$ of the expected location (and all of them with $10 \%$ of the expected location). However, there were many false peaks for the case of 10 samples and this may impair reliable detection. To determine if this was a real result, an additional 150 simulations were run (for a total of 200 different cases). For the data presented in the following section, the width of the filter will be swept to determine the impact of filter width on detection of the peaks. 


\subsubsection{Rougher Surface}

For the 36 grit surface, 20 unique cases of 10 averaged surfaces were investigated for the location of the Lorentz poles as listed in Table 5.2. Generally, all six of the peaks were visible within $5 \%$ of the expected location, but there were too many false peaks to allow for reliable detection, so this data will not be presented.

Since ten samples did not appear robust enough for reliable detection, the number of samples included was increased to 20. The 200 surfaces simulated allowed for a grouping of 10 different sets of 20 surfaces. To find the Lorentz poles from Tabel 5.2. The 20 surfaces were averaged, and the data was sampled at $-37^{\circ}$ (as illustrated in Figure 5.4). This data was then filtered using equation (5.1) for a variety of filter widths. The filtered data was then searched for the Lorentz poles listed in Table 5.2. The results of this are shown in Figures 5.9, and 5.10.

Figures 5.9 and 5.10 show all the cases of 20 averaged surfaces. Often, these figures illustrate that for a filter width of $1 / 12^{\text {th }}$ the space, all of the peaks are visible within $5 \%$ of the expected location. However, there are also some false peaks. Increasing filtering does reduce the number of false peaks (and often eliminates them) but at the cost of losing the $1.064 \mathrm{THz}$ peak and either the the merging of the 1.360 and $1.532 \mathrm{THz}$ peaks or the loss of the $1.532 \mathrm{THz}$ peak. The other three peaks near $0.808,1.980$, and $2.224 \mathrm{THz}$ are maintained. This may place a lower limit of 20 one-dimensional surface samples for reliable detection. 

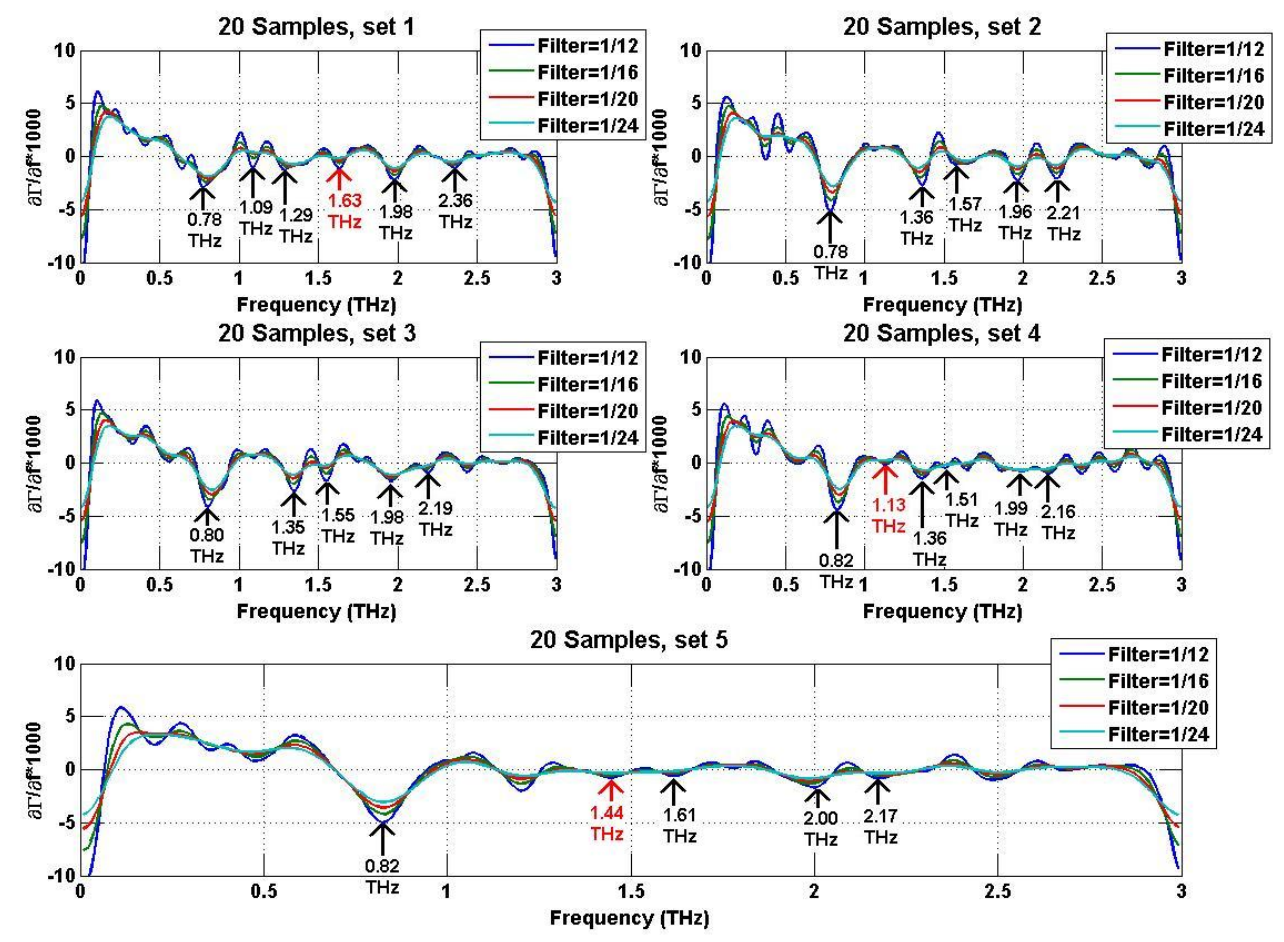

Figure 5.9: Derivative of smoothed data sampled at $-37^{\circ}$ from a homogeneous C-4 sample with 36 grit surface roughness. First five sets of twenty samples are averaged. The black and red arrows identify the peaks in Table 5.2 to within 5\% and $10 \%$ respectively. 

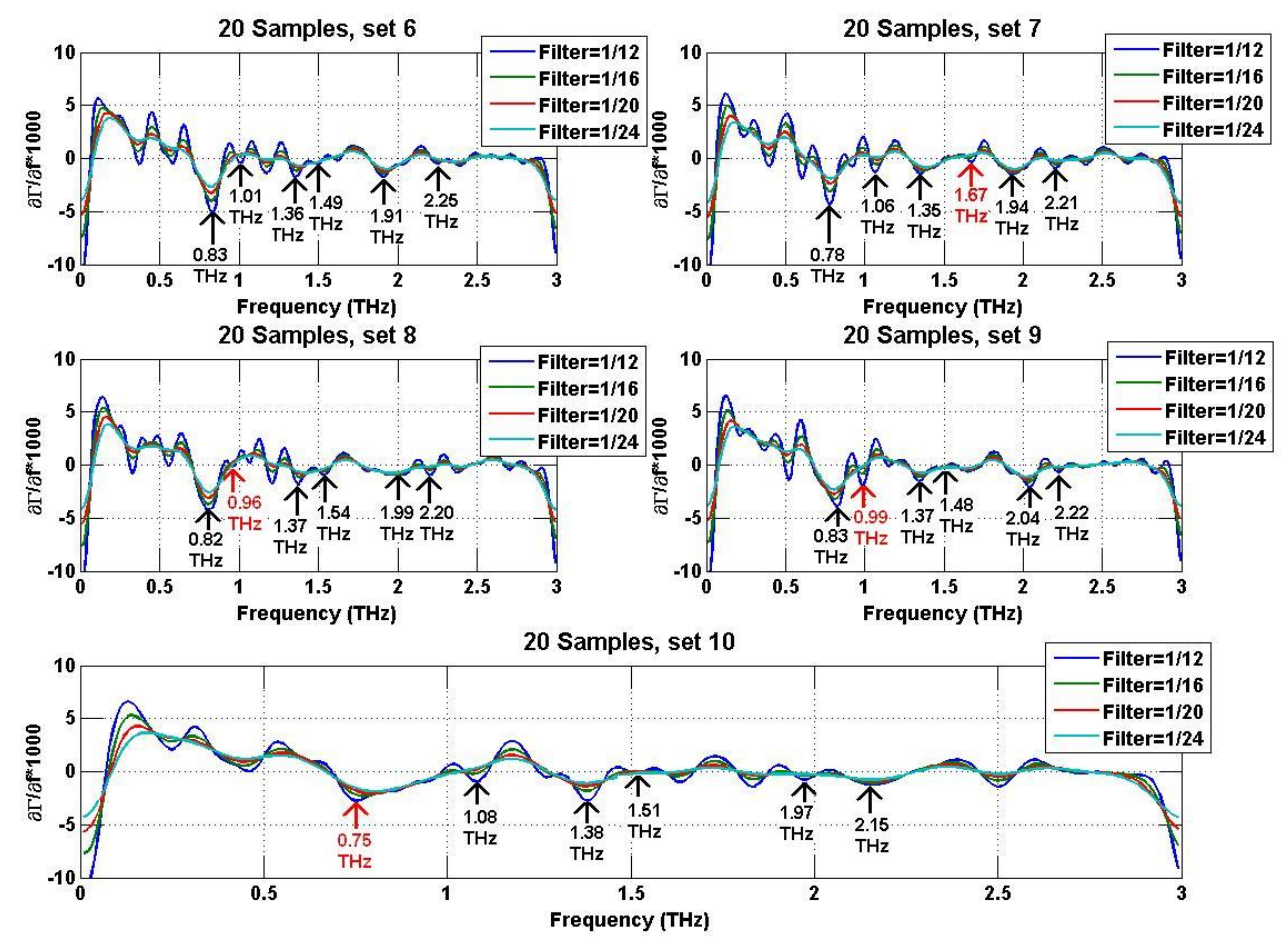

Figure 5.10: Derivative of smoothed data sampled at $-37^{\circ}$ from a homogeneous C-4 sample with 36 grit surface roughness. Second five sets of twenty samples are averaged. The black and red arrows identify the peaks in Table 5.2 to within $5 \%$ and $10 \%$ respectively. 

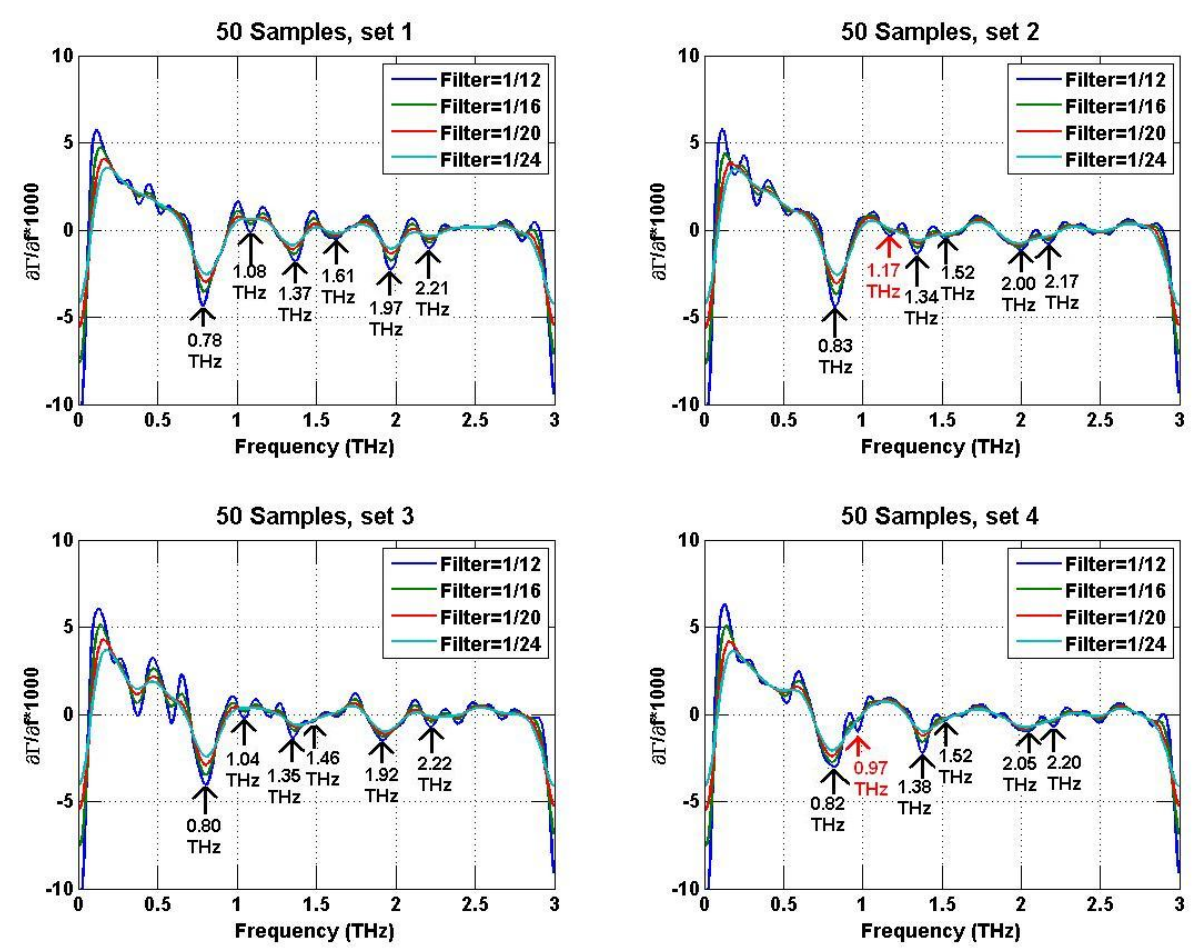

Figure 5.11: Derivative of smoothed data sampled at $-37^{\circ}$ from a homogeneous C-4 sample with 36 grit surface roughness. Fifty samples are averaged. The black and red arrows identify the peaks in Table 5.2 to within $5 \%$ and $10 \%$ respectively.

As the number of samples increases, the number of false peaks decreases, which relaxes the filtering requirements. Next, the same 200 surfaces allowed for 4 unique cases of 50 averaged surfaces to be investigated for the location of the Lorentz poles as listed in Table 5.2. The 50 surfaces were averaged, and the data was sampled at $-37^{\circ}$ (as illustrated in Figure 5.4). This data was then filtered using equation (5.1) for a variety of filter widths. The filtered data was then searched for the Lorentz poles listed in Table 5.2. The results of this are shown in Figure 5.11. 
Figure 5.11 shows the four cases of 50 averaged surfaces. For each of these cases all six of the peaks are seen, with most with $5 \%$ of the expected location. Only two of the peaks are within $10 \%$ of the expected location. As with the case of 20 sampled surface, the $1.064 \mathrm{THz}$ peak is often lost with increased filtering. Also, the $1.532 \mathrm{THz}$ peak is either lost or merged with the $1.360 \mathrm{THz}$ peak leaving only the $0.808,1.980$, and $2.224 \mathrm{THz}$ peaks unaltered.

\subsubsection{Smoother Surface}

For the 80 grit surface profile, the 20 unique cases of 10 averaged surfaces were investigated for the location of the Lorentz poles as listed in Table 5.2. Typically five of the six Lorentz poles were visible and within $5 \%$ of the expected location. However, as with the 36 grit profile, there are many false peaks which may prevent reliable detection. Therefore, it is likely that 10 samples is not enough for reliable detection.

To further explore the lower limit of surfaces to sample, the 10 unique cases of 20 averaged surfaces (sampled in the monostatic direction of $-37^{\circ}$ ) are sampled, and the data filtered using equation (5.1) with the width of the Gaussian shaped filter varied from $1 / 12^{\text {th }}$ to $1 / 24^{\text {th }}$ of the sample space to search for the Lorentz poles listed in Table 5.2. The results of this are shown in Figures 5.12 and 5.13.

Figures 5.12 and 5.13 show that at least five of the peaks typically seen within $5 \%$ of the expected value. The $1.064 \mathrm{THz}$ peak is often lost. When the filter 

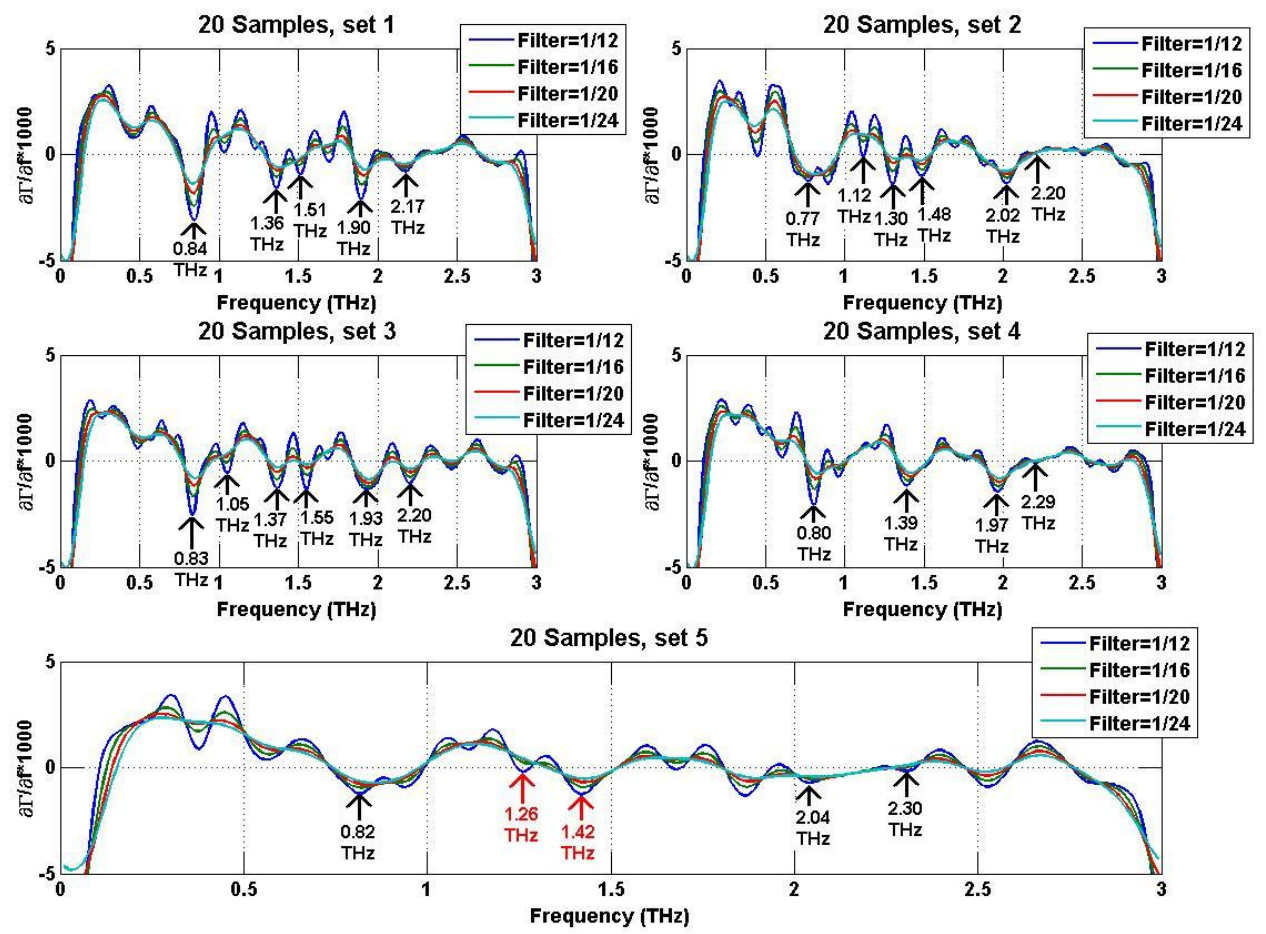

Figure 5.12: Derivative of smoothed data sampled at $-37^{\circ}$ from a homogeneous C-4 sample with 80 grit surface roughness. First five sets of twenty samples are averaged. The black and red arrows identify the peaks in Table 5.2 to within $5 \%$ and $10 \%$ respectively. 

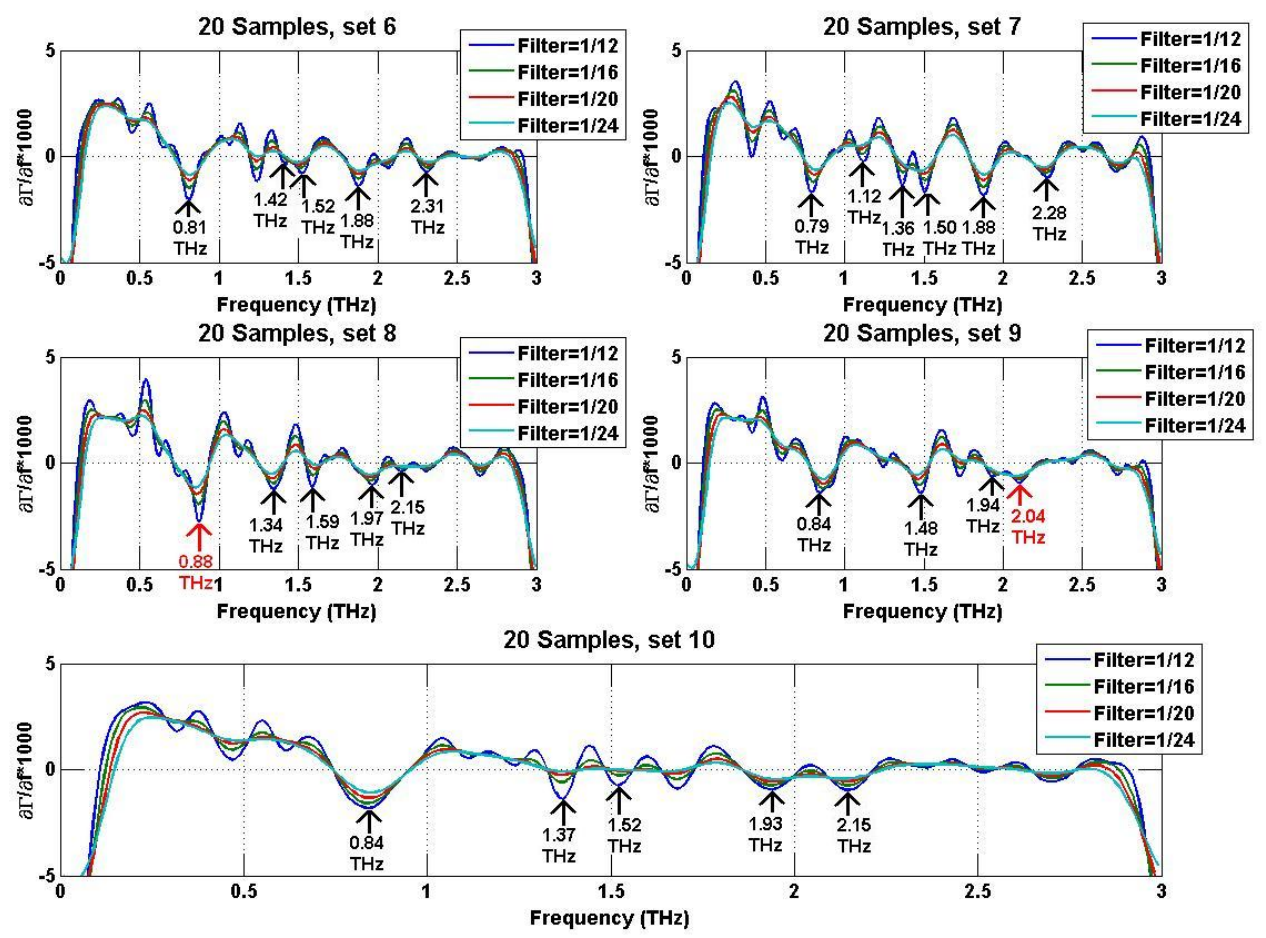

Figure 5.13: Derivative of smoothed data sampled at $-37^{\circ}$ from a homogeneous C-4 sample with 80 grit surface roughness. Second five sets of twenty samples are averaged. The black and red arrows identify the peaks in Table 5.2 to within $5 \%$ and $10 \%$ respectively. 
width is set to $1 / 12^{\text {th }}$ of the sample space, there are false peaks to obscure the actual peaks, but they are small and can be eliminated with increased filtering (i.e., narrower Gaussian filter) but the $1.064 \mathrm{THz}$ peak is frequently lost and the 1.360 and $1.532 \mathrm{THz}$ peaks are merged together (or the $1.532 \mathrm{THz}$ peak is lost) as was the case for the 36 grit profile.

When 50 surfaces are averaged and filtered, the results are little changed. At least five of the six peaks are visible and typically within $5 \%$ of the expected value. While false peaks are still visible, they are smaller and there are fewer of them. When the filtering is increased, the number of false peaks is reduced, but the $1.064 \mathrm{THz}$ peak is frequently lost, and the 1.360 and $1.532 \mathrm{THz}$ peaks are merged. The results of this are shown in Figure 5.14.

The case of 80 grit sandpaper typically lost the peak at $1.064 \mathrm{THz}$ (which is one of the smallest peaks) but the other peaks are typically visible, so detection would still be probable.

\subsection{Impact of System Noise}

Until this point, the focus has been on extracting the spectral peaks as measured from a rough surface in a simulator, which has infinite Signal-to-Noise Ratio (SNR). A real detection system will have noise, and that must be considered in this analysis. For the 200 cases run for the Monte-Carlo analysis used in the prior section, varying amounts of random frequency domain noise will be added 

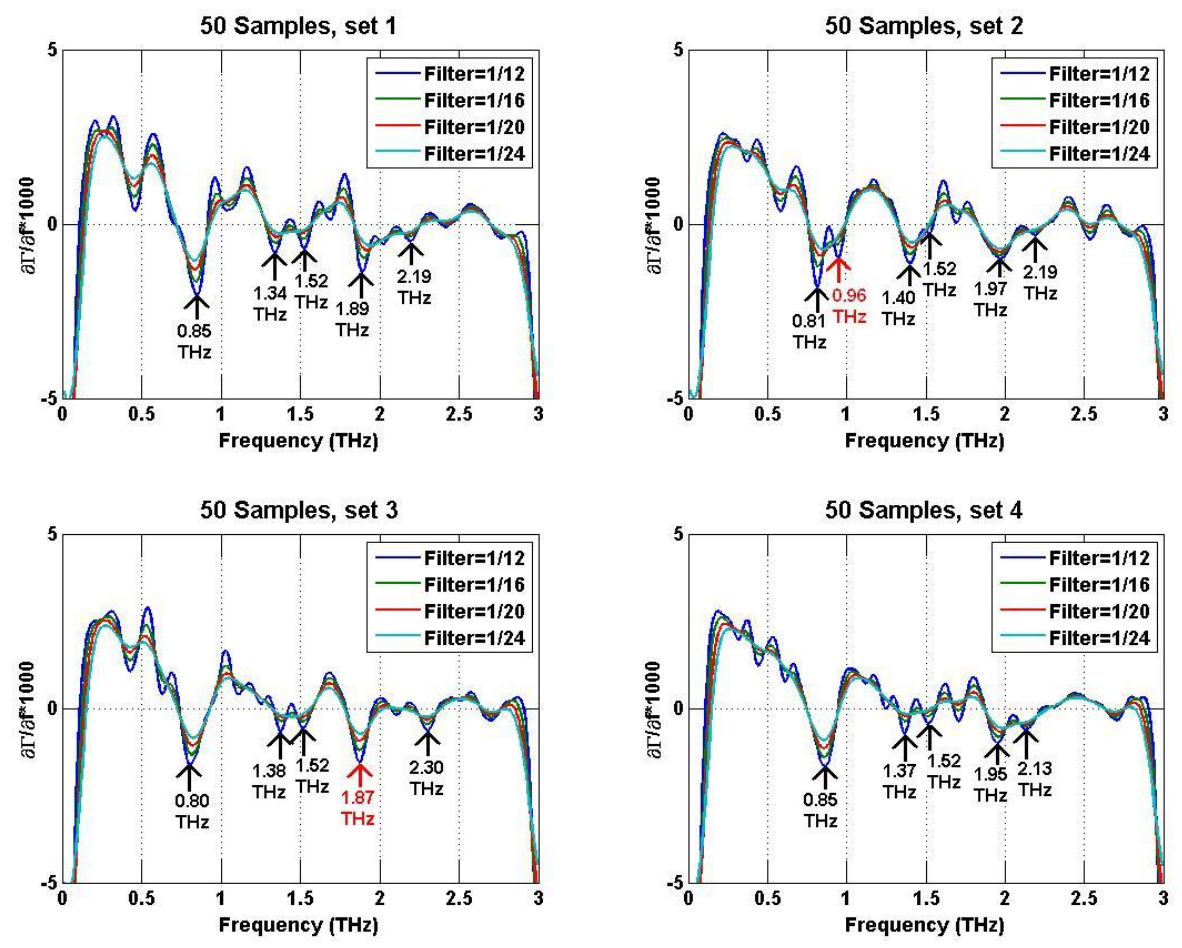

Figure 5.14: Derivative of smoothed data sampled at $-37^{\circ}$ from a homogeneous C-4 sample with 80 grit surface roughness. Fifty samples are averaged. The black and red arrows identify the peaks in Table 5.2 to within $5 \%$ and $10 \%$ respectively. 
to the power data from each simulated surface to give an SNR of $0 \mathrm{~dB}, 10 \mathrm{~dB}$, and $20 \mathrm{~dB}$. These cases with noise will then be averaged over 20 and 50 surfaces to see what impact the noise may have on the detection of the peaks. The width of the Gaussian filter is fixed at a width of $1 / 12^{\text {th }}$ of the sample space as this case is most susceptible to system noise.

\subsubsection{Rough Surface}

Figure 5.15 shows many cases of 50 averaged 36 grit surfaces with noise added. There is almost no change in the spectral shape for the case of 20dB SNR. When the SNR is reduced to $10 \mathrm{~dB}$, the spectral signature begins to be altered, but not so much as to obscure detection. However, when the SNR is degraded to 0dB, the spectral signature is significantly changed, and is lost.

When only 20 surfaces are made available for averaging, the effect noise has on the extracted spectral signature is not changed. That is, for 20 surfaces, there is no impact on the extracted signature if a $20 \mathrm{~dB}$ SNR is maintained. A $10 \mathrm{~dB}$ SNR begins to change the spectral signature, but not enough to hide the peaks. However, for a $0 \mathrm{~dB}$ SNR, the spectral signature is totally lost. As this is the same result as shown for 50 samples, no illustration is given. 


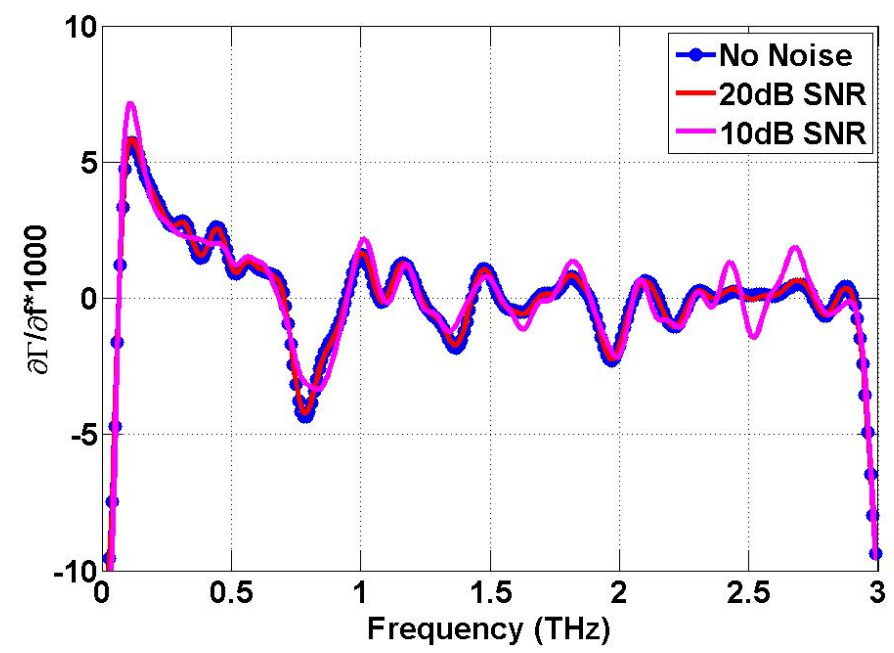

Figure 5.15: Derivative of smoothed data sampled at $-37^{\circ}$ from a homogeneous C-4 sample with 36 grit surface roughness. Fifty samples were averaged. Noise was added to get an SNR of $10 \mathrm{~dB}$ and $20 \mathrm{~dB}$.

\subsubsection{Smoother Surface}

The simulated data for the 80 grit surface had enough noise added to each sample to achieve the desired 0,10, and 20dB SNR. Figure 5.16 shows the results of many cases with 50 averaged surfaces to find the location of the Lorentz poles in Table 5.2. As seen for the case of the 36 grit surface, an SNR of 20dB did not alter the spectral signature, an SNR of 10dB began to alter the spectral signature, and an SNR of 0dB totally masked the spectral signature.

When only 20 surfaces are available for averaging, the results are little changed. When a $20 \mathrm{~dB}$ SNR is maintained, the spectral signature is unchanged. When a $10 \mathrm{~dB}$ SNR is maintained, the spectral signature is beginning to change, but not enough to obscure the peaks. For an SNR of $0 \mathrm{~dB}$, the spectral signature is 


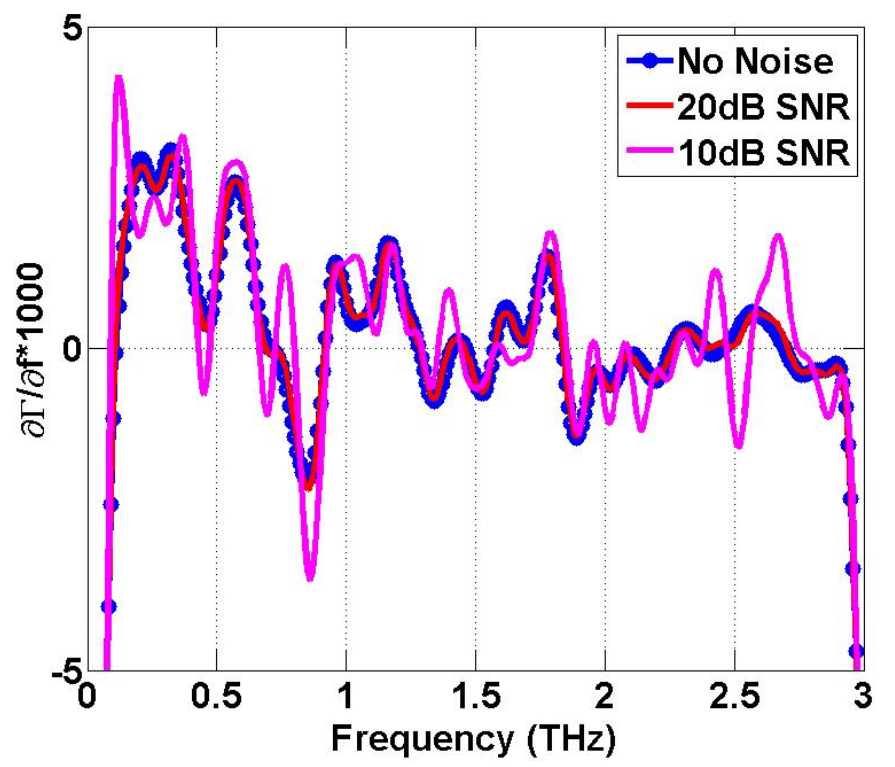

Figure 5.16: Derivative of smoothed data sampled at $-37^{\circ}$ from a homogeneous C-4 sample with 80 grit surface roughness. Fifty samples were averaged. Noise was added to get an SNR of $10 \mathrm{~dB}$ and $20 \mathrm{~dB}$.

completely lost. Since this is the same result as the case with 50 averages, no illustration is presented.

For both of the simulated surfaces (i.e., 36 grit and 80 grit), the addition of noise had little impact on the extracted spectral signature as long as the SNR was $10 \mathrm{~dB}$ or better. Once the SNR was degraded to $0 \mathrm{~dB}$, then the peaks could not be reliably extractable. For this reason, a 10dB SNR should be maintained in a measurement system. 


\subsection{Changing the Angle of Incidence}

The prior sections all assumed the incident wave arrived at $-37^{\circ}$ as measured from the normal. This section will explore the effect of the incident angle on the extraction of the spectral signature. Each of the incident angles $\left(-10^{\circ},-30^{\circ},-50^{\circ}\right.$, and $-70^{\circ}$ measured from the surface normal) will use 50 samples for the MonteCarlo simulations. Cases will be run for the surface roughness profile of 36 grit sandpaper and 80 grit sandpaper. Also, the impact of noise will be investigated for each surface and angle of incidence as well.

As illustrated for an incident angle of $-37^{\circ}, 20$ samples showed the Lorentz poles as well as the case of 50 sampled surfaces. Because of this, only the data for 50 averaged surfaces is presented.

As described earlier in this chapter, the averaged data will be investigated for the location of the Lorentz poles as listed in Table 5.2. First, the data was sampled in the back scattered direction at either $-10^{\circ},-30^{\circ},-50^{\circ}$, or $-70^{\circ}$, depending on the incident angle. Then enough noise is added to each of the 50 samples to get an SNR of $20 \mathrm{~dB}, 10 \mathrm{~dB}$ or $0 \mathrm{~dB}$. The data is then averaged over the appropriate number of averages and this is filtered using Equation (5.1) with the width of the filter being swept to help determine the optimum filter size. 


\subsubsection{Rough Surface Variation With Angle of Incidence}

For the 36 grit surface, when the angle of incidence is varied from $-10^{\circ}$ to $-70^{\circ}$ and the data is sampled in the backscatter direction (for a monostatic detection regime) it is determined that all six of the peaks can be extracted within $5 \%$ of the expected value with a filter width of $1 / 12^{\text {th }}$ the frequency space if the angle of incidence is restricted to $-50^{\circ}$ or less. This is because the power from an incident angle of $-50^{\circ}$ is beginning to drop. For an incident angle of $-70^{\circ}$, the data is being sampled on the edge of the Radar Cross Section (RCS) curve where the power is rapidly falling off. This low power level negatively impacted the ability to detect the $1.064,1.532$, and the $2.224 \mathrm{THz}$ peaks. Therefore, no data is presented for an incident angle of $-70^{\circ}$.

Table 5.3 shows the location of the extracted peaks vs. the amount of filtering. As seen with the $37^{\circ}$ data, less filtering shows more peaks, but with increased false peaks. More filtering eliminates the false peaks at the cost of the $1.064 \mathrm{THz}$ peak. The $1.360 \mathrm{THz}$ peak is either lost or merged with the $1.532 \mathrm{THz}$ peak.

When noise is added to each sample, the extracted signature is unchanged for a $20 \mathrm{~dB}$ SNR (as seen in the $-37^{\circ}$ case). The signature begins to change at $10 \mathrm{~dB}$ SNR, but not enough to obscure detection. At $0 \mathrm{~dB}$ SNR, the signature is totally lost. No plots are shown as the results are identical to the $-37^{\circ}$ case. 
Table 5.3: Location (in $\mathrm{THz}$ ) of extracted Lorentz poles for data sampled in the backscatter direction from a homogenous C-4 sample with the surface roughness of 36 grit sandpaper. Data reported for peaks within $5 \%$ of the expected value.

\begin{tabular}{|c|c|c|c|c|c|c|c|}
\hline $\begin{array}{c}\text { Incident } \\
\text { Angle }\end{array}$ & $\begin{array}{l}\text { Filter } \\
\text { Width }\end{array}$ & $\begin{array}{c}0.808 \\
\mathrm{THz} \\
\text { Peak }\end{array}$ & $\begin{array}{c}1.064 \\
\mathrm{THz} \\
\text { Peak }\end{array}$ & $\begin{array}{c}1.360 \\
\mathrm{THz} \\
\text { Peak }\end{array}$ & $\begin{array}{c}1.532 \\
\mathrm{THz} \\
\text { Peak }\end{array}$ & $\begin{array}{c}1.980 \\
\mathrm{THz} \\
\text { Peak }\end{array}$ & $\begin{array}{c}2.224 \\
\mathrm{THz} \\
\text { Peak }\end{array}$ \\
\hline \multirow{4}{*}{$-10^{\circ}$} & $1 / 12^{\text {th }}$ & 0.83 & 1.06 & 1.35 & 1.52 & 2.02 & 2.19 \\
\hline & $1 / 16^{t h}$ & 0.83 & 1.06 & 1.35 & 1.51 & 2.02 & 2.20 \\
\hline & $1 / 20^{t h}$ & 0.83 & 1.05 & 1.36 & 1.51 & 2.00 & 2.21 \\
\hline & $1 / 24^{\text {th }}$ & 0.83 & - & 1.38 & - & 1.99 & 2.21 \\
\hline \multirow{4}{*}{$-30^{\circ}$} & $1 / 12^{\text {th }}$ & 0.85 & 1.07 & 1.31 & 1.49 & 1.95 & 2.20 \\
\hline & $1 / 16^{t h}$ & 0.84 & 1.08 & 1.30 & 1.51 & 2.00 & 2.19 \\
\hline & $1 / 20^{t h}$ & 0.83 & 1.08 & 1.31 & 1.50 & 2.00 & 2.18 \\
\hline & $1 / 24^{t h}$ & 0.83 & - & 1.31 & 1.50 & 2.00 & 2.18 \\
\hline \multirow{4}{*}{$-50^{\circ}$} & $1 / 12^{\text {th }}$ & 0.79 & - & 1.39 & 1.57 & 2.03 & 2.26 \\
\hline & $1 / 16^{t h}$ & 0.80 & - & 1.39 & 1.56 & 2.03 & 2.26 \\
\hline & $1 / 20^{t h}$ & 0.80 & - & 1.40 & - & 2.02 & 2.25 \\
\hline & $1 / 24^{t h}$ & 0.80 & - & 1.40 & - & 2.01 & 2.25 \\
\hline
\end{tabular}

\subsubsection{Smoother Surface Variation With Angle of Incidence}

For the 80 grit surface, when the angle of incidence is varied from $-10^{\circ}$ to $-70^{\circ}$ and the data is sampled in the backscatter direction (for a monostatic detection regime) it is seen that all at least five of the six of the peaks can be extracted and are within $5 \%$ of the expected value if the angle of incidence is restricted to $-50^{\circ}$ or less for the same reason as for the rougher surface. A summary of the extracted peaks over angle of incidence is given in Table 5.4. As with the rougher surface, if the filter width is swept from $1 / 12^{\text {th }}$ of the sample space to $1 / 24^{\text {th }}$ the 
Table 5.4: Location (in THz) of extracted Lorentz poles for data sampled in the backscatter direction from a homogenous C-4 sample with the surface roughness of 80 grit sandpaper. Data reported for peaks within $5 \%$ of the expected value. Red values are within $10 \%$.

\begin{tabular}{|c|c|c|c|c|c|c|c|}
\hline $\begin{array}{l}\text { Number } \\
\text { of Sur- } \\
\text { faces }\end{array}$ & $\begin{array}{l}\text { Filter } \\
\text { Width }\end{array}$ & $\begin{array}{l}0.808 \\
\mathrm{THz} \\
\text { Peak }\end{array}$ & $\begin{array}{c}1.064 \\
\mathrm{THz} \\
\text { Peak }\end{array}$ & $\begin{array}{l}1.360 \\
\mathrm{THz} \\
\text { Peak }\end{array}$ & $\begin{array}{l}1.532 \\
\mathrm{THz} \\
\text { Peak }\end{array}$ & $\begin{array}{l}1.980 \\
\mathrm{THz} \\
\text { Peak }\end{array}$ & $\begin{array}{c}2.224 \\
\mathrm{THz} \\
\text { Peak }\end{array}$ \\
\hline \multirow{4}{*}{$-10^{\circ}$} & $1 / 12^{t h}$ & 0.82 & - & 1.32 & 1.54 & 1.92 & 2.21 \\
\hline & $1 / 16^{t h}$ & 0.82 & - & - & 1.53 & 1.91 & 2.22 \\
\hline & $1 / 20^{t h}$ & 0.82 & - & - & 1.50 & 1.92 & - \\
\hline & $1 / 24^{t h}$ & 0.82 & - & - & 1.50 & 1.93 & - \\
\hline \multirow{4}{*}{$-30^{\circ}$} & $\overline{1} 1 / 12^{\text {th }}$ & 0.84 & 1.05 & 1.46 & 1.60 & 1.92 & 2.18 \\
\hline & $1 / 16^{t h}$ & 0.84 & - & - & 1.60 & 1.92 & 2.18 \\
\hline & $1 / 20^{t h}$ & 0.84 & - & - & 1.59 & 1.92 & 2.18 \\
\hline & $1 / 24^{t h}$ & 0.84 & - & - & 1.59 & 1.92 & 2.17 \\
\hline \multirow{4}{*}{$-50^{\circ}$} & $1 / 12^{t h}$ & 0.85 & - & 1.38 & 1.50 & 1.97 & 2.20 \\
\hline & $1 / 16^{\text {th }}$ & 0.85 & - & 1.38 & 1.50 & 1.98 & 2.21 \\
\hline & $1 / 20^{t h}$ & 0.85 & - & 1.39 & - & 1.99 & 2.21 \\
\hline & $1 / 24^{t h}$ & 0.85 & - & 1.40 & - & 2.00 & 2.21 \\
\hline
\end{tabular}

sample space the number of false peaks is reduced, however the $1.064 \mathrm{THz}$ peaks is lost, the 1.360 and $1.532 \mathrm{THz}$ peaks are merged (or the $1.532 \mathrm{THz}$ peaks is lost). As with the rougher surface, no data is presented for an incident angle of $-70^{\circ}$ since there is insufficient power for reliable detection.

When the filter width is fixed at $1 / 12^{\text {th }}$ of the space, and enough noise is added to achieve an SNR of 0, 10, and $20 \mathrm{~dB}$, the spectral signature is still seen as long as an SNR of $10 \mathrm{~dB}$ is maintained. This is the same results as the $-37^{\circ}$ data. 


\subsection{Conclusion}

In this chapter the impact of rough surface scattering on the spectral signature of the explosive C-4 was investigated. While the rough surface does allow for detection of the reflected wave at the same location as the source of the wave (i.e., a monostatic detection regime), the rough surface scattering effects will mask the spectral signature without sufficient filtering. This chapter introduced a new way to effectively filter the data and make it possible to extract the spectral signature, even in the presence of noise (providing a 10dB SNR is maintained) with as few as 20 samples, with a limited number of false peaks. The false peaks could typically be eliminated with more filtering, but some of the actual Lorentz poles may be lost. The $0.808,1.980$, and $2.224 \mathrm{THz}$ peaks are typically maintained and the 1.360 and $1.532 \mathrm{THz}$ peaks were often merged together (or the $1.532 \mathrm{THz}$ peak was lost). This is regardless of the angle of incidence of the wave. However, since the amount of power detected at large angles of incidence (at $-70^{\circ}$ for the 36 grit and 80 grit surfaces), it is recommended that the angle of incidence be restricted to approximately $-50^{\circ}$ or less with 20 surface samples or more for a finite width beam. 


\section{Chapter 6}

\section{Effect of Granular Scattering}

It was determined that the spectral signature can be extracted only if rough surface scattering is considered. Next, only the granular nature of the explosive is investigated. In this chapter, the effect that only the granular nature of the media has on the extracted spectral signature is explored. The methods introduced in the prior chapter to extract the Lorentz poles will be tested.

\subsection{Modeling the Granular Nature of Composition-4}

To understand the effect granular scattering may have on the extracted spectral signature, several simulation cases are run with a smooth surface interface and spheres to approximate the grains. The composition of C-4 is typically $90 \%$ RDX and $10 \%$ polyisobutylene $\left(\epsilon_{r}=3\right)$ as shown in Table 2.2 .

Work done by Borne indicates that RDX grains can be in the range of 100 to $800 \mu \mathrm{m}$ with a large portion of the grain sizes being under $300 \mu \mathrm{m}$ [66]. Also,

intragranular voids are engineered to be small in size and number (fractional 
Table 6.1: Simulation cases run to study granular scattering.

\begin{tabular}{|c||c|c|c|}
\hline Case & Grain Radius $(\mu \mathrm{m})$ & $\begin{array}{c}\text { Calculated Filler } \\
\text { Radius }(\mu \mathrm{m})\end{array}$ & $\begin{array}{c}\text { Air Void } \\
\text { Radius }(\mu \mathrm{m})\end{array}$ \\
\hline 1 & 100 & 39 & 8 \\
\hline 2 & 300 & 116 & 20 \\
\hline
\end{tabular}

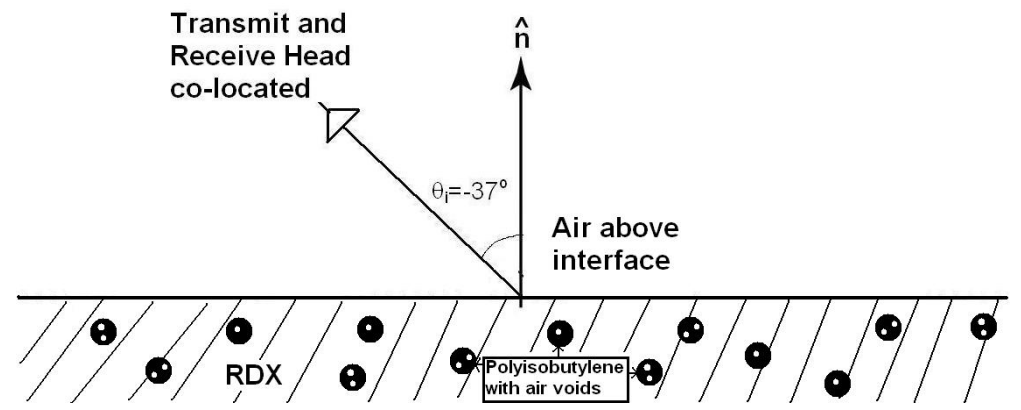

Figure 6.1: Schematic representation of granular simulations.

volumes of $0.44 \%$ or less) to maintain stability of the explosive while transporting it. The polyisobutylene filler that binds the RDX grains together has air voids to support the expanding explosive wave front with a fractional volume of $2-6 \%$.

As introduced in Chapter 4, the C-4 mixture will be modeled as a background of the dispersive C-4 media with a $10 \%$ fractional volume of polyisobutylene spherical voids. The radius of the voids is determined using Equation (4.1). Then the polyisobutylene is filled with air voids having a radius of $8-20 \mu \mathrm{m}$ and a fractional volume of $3.5 \%$. No intragranular voids are added to the $\mathrm{C}-4$ as the fractional volume of these voids is so small. Table 6.1 summarizes the simulation cases run. Figure 6.1 illustrates the simulation setup.

To perform the simulations for the cases listed in Table 6.1, the number of 
polyisobutylene scatterers is calculated using Equation (4.2). As the simulation is two-dimensional, the simulation is a slice of the C-4. Therefore, the scatterers are actually cylinders. The centers of these scatterers is then determined using a random number generator taking care to make sure no centers are repeated and that no scatterers overlap. Once the C-4 simulation space is filled with polyisobutylene, the air scatterers are added to only the polyisobutylene using the same procedure taking care that the air voids do not overlap and that they occur only in the polyisobutylene. The FDTD simulation is run on this case, and the far-field data is recorded. The centers of both the polyisobutylene and air scatterers are then redetermined using a random number generator for a total of 50 different cases (with a surface length of $10 \mathrm{~cm}$, discretized at 40 cells per free space wavelength at $1.5 \mathrm{THz}$ ) as needed for the Monte-Carlo analysis. A monostatic (i.e., transmitter and receiver are co-located) detection regime is assumed for this simulation to more closely match a possible real-world application. The average of these simulation cases is then reported. 


\subsection{Impact of the Granular Nature of Composition-4 Spectral Signa- ture: No System Noise}

\subsubsection{Small Grain Size}

The FDTD simulations were run with an angle of incidence of $-37^{\circ}$ and a grain size of $100 \mu \mathrm{m}$. The FDTD results include far-field data at all receive angles and frequencies. The FDTD RCS data is averaged over a 5 degree window centered at a receive angle of $-37^{\circ}$ (relative the normal) to approximate a receiving antenna with a finite width main lobe. The amount of reflected power due to the granular scattering in the backscattered direction is small and increases as the frequency increases. This makes sense as the size of the scatterers is increasing with respect to the wavelength as the frequency increases. Therefore, as was the case for a $-70^{\circ}$ incident angle with a homogenous material, it is difficult to extract the spectral signature.

The data sampled around $-37^{\circ}$ is smoothed using Equation (5.1). The derivative of this smoothed data is then plotted in Figure 6.2 for the average of 50 samples with the standard deviation (i.e., the filter width) of the gaussian shaped filter swept from $1 / 12^{\text {th }}$ to $1 / 24^{\text {th }}$ the sample space.

Figure 6.2 shows that four of the six peaks can be extracted from the cases run with 50 averaged surfaces. The peaks that are detected are within $5 \%$ of the expected value. In addition, the number of false peaks is high, even with increased 


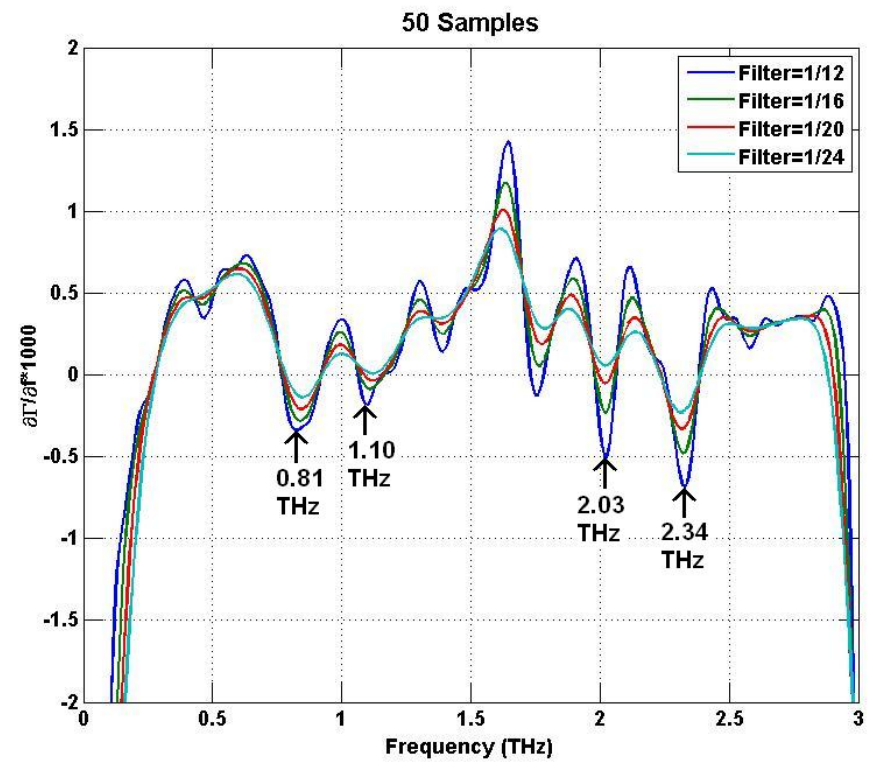

Figure 6.2: Derivative of smoothed data sampled at $-37^{\circ}$ from a background of C-4 with a smooth interface and a grain size of $100 \mu \mathrm{m}$. Fifty samples were averaged. The black arrows identify peaks in Table 5.2. to within $5 \%$.

filtering. However, the lack of depth of all the peaks may make detection very difficult if relying only on the granular scattering to provide energy in the nonspecular direction. While not presented, the same was found to be true if the number of samples in the average was restricted to 20 .

\subsubsection{Larger Grain Size}

The FDTD simulations were run with an angle of incidence of $-37^{\circ}$ and a grain size of $300 \mu \mathrm{m}$. The FDTD results include far-field data at all receive angles and frequencies. The FDTD RCS data is averaged over a 5 degree window centered at a receive angle of $-37^{\circ}$ (relative the normal) to approximate a receiving antenna 


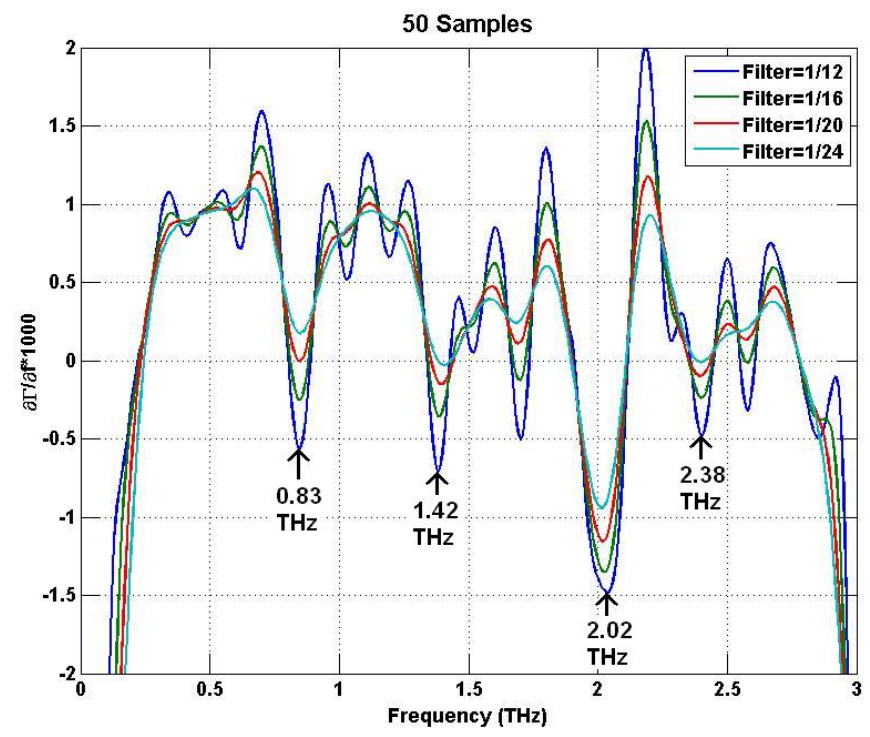

Figure 6.3: Derivative of smoothed data sampled at $-37^{\circ}$ from a background of C-4 with a smooth interface and a grain size of $300 \mu \mathrm{m}$. Fifty samples were averaged. The black arrows identify peaks in Table 5.2. to within $5 \%$.

with a finite width main lobe. The amount of reflected power due to the granular scattering in the backscattered direction is small and increases as the frequency increases. This makes sense as the size of the scatterers is increasing with respect to the wavelength as the frequency increases. Therefore, as was the case for a smaller grain sizes, it is difficult to extract the spectral signature.

The data sampled around $-37^{\circ}$ is smoothed using Equation (5.1). The derivative of this smoothed data is then plotted in Figure 6.3 for the average of 50 samples with the standard deviation (i.e., the filter width) of the gaussian shaped filter swept from $1 / 12^{\text {th }}$ to $1 / 24^{\text {th }}$ the sample space.

Figure 6.3 shows that four of the six peaks can be extracted from the cases 
run with 50 averaged surfaces. The peaks that are detected are within $5 \%$ of the expected value. In addition, the number of false peaks is not significantly reduced with increased filtering. However, the lack of depth of all the peaks may make detection very difficult if relying only on the granular scattering to provide energy in the non-specular direction. While not presented, the same was found to be true if the number of samples in the average was restricted to 20 .

\subsection{Impact of the Granular Nature of Composition-4 Spectral Signa- ture: With System Noise}

Until this point, the focus has been on extracting the spectral peaks as measured from a sample with a granular nature in a simulator, which has infinite Signalto-Noise Ratio (SNR). A real detection system will have noise, and that must be considered in this analysis. For the 50 cases run for the Monte-Carlo analysis used in the prior section, varying amounts of random noise will be added to each simulation case to give an SNR of $0 \mathrm{~dB}, 10 \mathrm{~dB}$, and $20 \mathrm{~dB}$. These cases with noise will then be averaged over 50 surfaces to see what impact the noise may have on the detection of the peaks.

\subsubsection{Small Grain Size}

The results for a $100 \mu \mathrm{m}$ grain size material with $-37^{\circ}$ angle of incidence with noise is shown in Figure 6.4. 


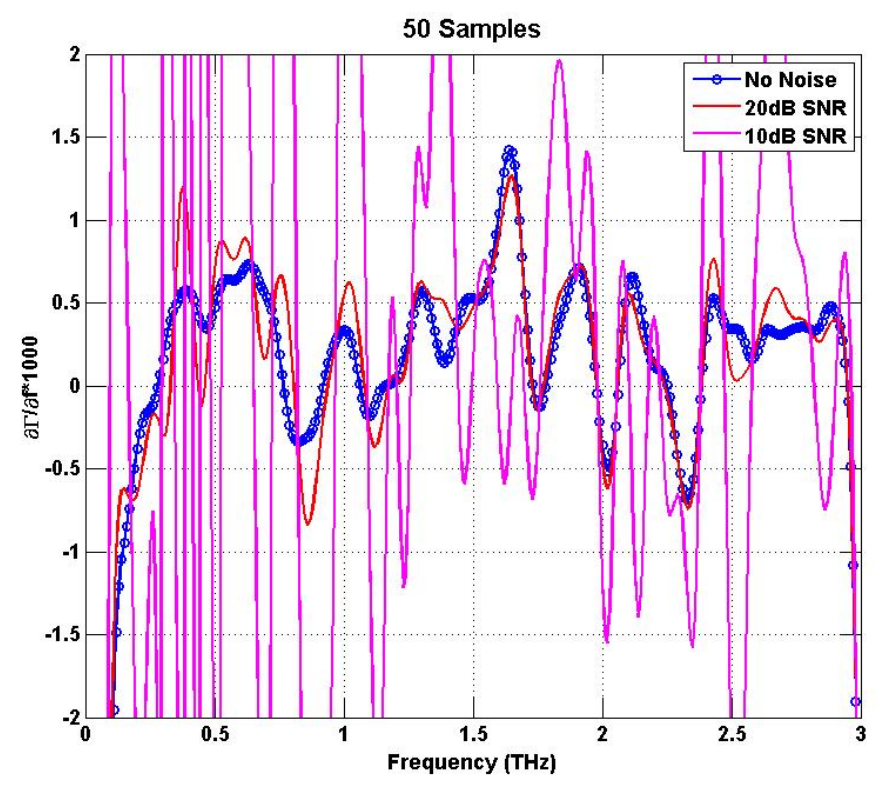

Figure 6.4: Derivative of smoothed data sampled at $-37^{\circ}$ from a background of C-4 with a smooth interface and a grain size of $100 \mu \mathrm{m}$. Fifty samples were averaged. Noise was added to get an SNR of $10 \mathrm{~dB}$ and $20 \mathrm{~dB}$.

As shown in Figure 6.4, when enough noise is added to the simulated FDTD data to attain an SNR of $20 \mathrm{~dB}$, there is virtually no change in the extracted Lorentz poles. When enough noise is added to attain an SNR of $10 \mathrm{~dB}$, the spectral signature is beginning to be distorted, but the extracted peaks are still visible. When enough noise is added to attain an SNR of $0 \mathrm{~dB}$, the spectral signature is totally changed with the peaks shifting around in frequency and many false peaks appearing. Therefore, an SNR of greater than $10 \mathrm{~dB}$ should be maintained for reliable detection. The same is true if only 20 samples is used in the average, and therefore the data is not presented. 


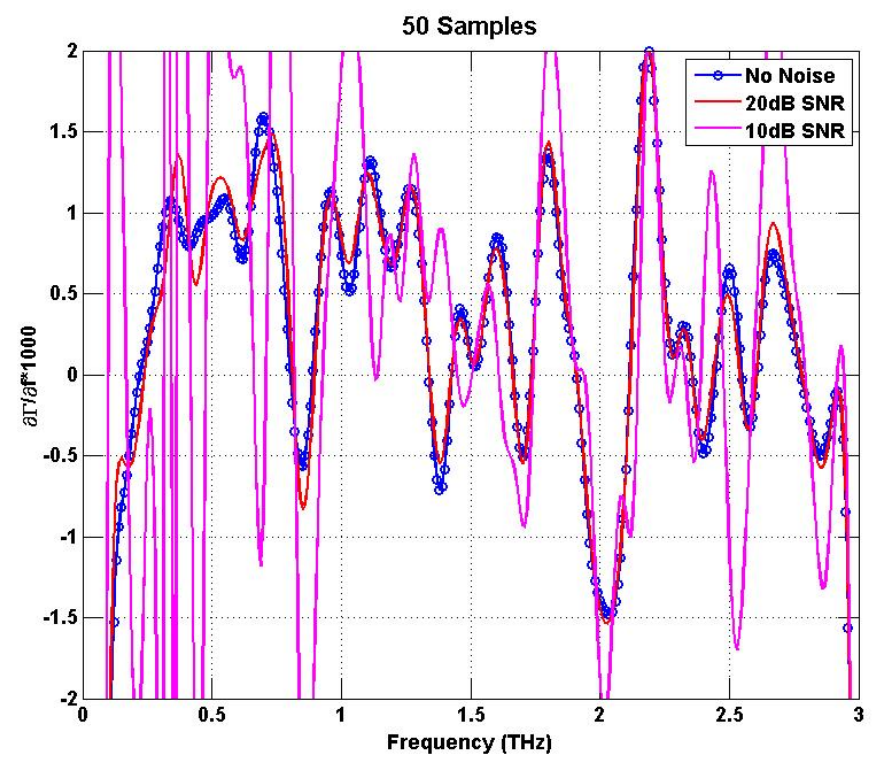

Figure 6.5: Derivative of smoothed data sampled at $-37^{\circ}$ from a background of C-4 with a smooth interface and a grain size of $300 \mu \mathrm{m}$. Fifty samples were averaged. Noise was added to get an SNR of $10 \mathrm{~dB}$ and $20 \mathrm{~dB}$.

\subsubsection{Larger Grain Size}

The results for a $300 \mu \mathrm{m}$ grain size material with $-37^{\circ}$ angle of incidence with noise is shown in Figure 6.5.

As shown in Figure 6.5, when enough noise is added to the simulated FDTD data to attain an SNR of $20 \mathrm{~dB}$, there is virtually no change in the extracted Lorentz poles. When enough noise is added to attain an SNR of $10 \mathrm{~dB}$, the spectral signature is beginning to be distorted, but the extracted peaks are still visible. When enough noise is added to attain an SNR of $0 \mathrm{~dB}$, the spectral signature is totally changed with the peaks shifting around in frequency and many false peaks appearing. Therefore, an SNR of greater than $10 \mathrm{~dB}$ should be 
maintained for reliable detection. The same is true if only 20 samples is used in the average, and therefore the data is not presented.

\subsection{Other Angles of Incidence}

Other angles of incidence were simulated and the data processed. As seen in Chapter 5, there was little dependence on the incident angle. The only insight here is that for angles of incidence over $-50^{\circ}$, the reflected power drops rapidly. Therefore, as in the case of just rough surface scattering, the angle of incidence should be kept between $0^{\circ}$ and $-50^{\circ}$. It should again be stated that this survey was restricted to sampling power in the backscattered direction (i.e., a monostatic detection regime).

\subsection{Conclusion}

This chapter looked at the effect of granular scattering on the extracted spectral signature of C-4. It is possible to extract some of the Lorentz poles (the bigger peaks around 0.808 and $1.980 \mathrm{THz}$ and the higher frequency peak at $2.244 \mathrm{THz}$ ). However, the amount of power reflected is small. Also, the smaller peaks (1.064 and $1.532 \mathrm{THz}$ ) are generally lost to the size related scattering. So, it is not possible to reliably extract all of the peaks when relying only on the back scattered energy generated by only the granular scattering.

When noise was added to the simulated data, the spectral signature did not 
change if greater than a 10dB SNR was maintained. Therefore, greater than a 10dB SNR should be maintained.

Practically speaking, it is probably not possible to extract the spectral peak relying only on the granular scattering as the amount of reflected power is lower than that from just the rough surface scattering. It is encouraging, however, that the back scattered energy from the granular scattering is low. This implies that the assumption made in Chapter 5 about the surface roughness dominating the scattering, is correct. If this is true, then it should be possible to extract the spectral signature if the model includes a rough surface and the granular scatterers. This idea will be explored in the next chapter. 


\section{Chapter 7}

\section{Combined Effect of Random Media Interface and Granular Scattering}

It has been determined that the rough surface scattering has a dominant effect on the spectral signature extraction. Also, the granular scattering does not provide enough non-specular energy to allow for reliable detection. In this chapter, the combined effect that the rough surface and the granular nature of the media has on the extracted spectral signature is explored. The methods introduced in the prior chapters to extract the Lorentz poles will be tested. The amount of filtering required to eliminate the false peaks from the spectral signature will be explored.

\subsection{Modeling the Rough Surface and Granular Nature of Composition- 4}

To study the effect of a rough surface and the granular nature of the explosive on the spectral signature of the C-4, an FDTD simulation is computed assuming a rough surface for the C-4 material interface with statistics of either 36 grit or 80 grit sandpaper (as reported in Table 5.1) [60]. 
Table 7.1: Simulation cases run to study granular scattering.

\begin{tabular}{|c||c|c|c|c|}
\hline Case & Surface & Grain Radius $(\mu \mathrm{m})$ & $\begin{array}{c}\text { Calculated Filler } \\
\text { Radius }(\mu \mathrm{m})\end{array}$ & $\begin{array}{c}\text { Air Void Radius } \\
(\mu \mathrm{m})\end{array}$ \\
\hline 1 & 36 Grit & 100 & 39 & 8 \\
\hline 2 & 36 Grit & 300 & 116 & 20 \\
\hline 3 & 80 Grit & 100 & 39 & 8 \\
\hline 4 & 80 Grit & 300 & 116 & 20 \\
\hline
\end{tabular}

In addition to the rough surface, the granular scatterers discussed in the prior chapter are added to the simulation space as well. Recall the composition of C-4 is typically $90 \%$ RDX and $10 \%$ polyisobutylene $\left(\epsilon_{r}=3\right)$ as shown in Table 2.2 . There are air voids within the polyisobutylene with a fractional volume of $2-6 \%$, with no air voids being modeled in the C-4 [66]. Because of the large fractional volume of the $\mathrm{C}-4$, this is used as the background with the polyisobutylene used as the scatterers with the size of the scatterers determined using Equation (4.1). The polyisobutylene is filled with air voids having a radius of 8 or $20 \mu \mathrm{m}$ and a total fractional volume of $3.5 \%$. Table 7.1 summarizes the simulation cases run.

To perform the simulations for the cases listed in Table 7.1, the number of polyisobutylene scatterers is calculated using Equation (4.2). The centers of these scatterers is then determined using a random number generator taking care to make sure no centers are repeated and that no scatterers overlap. Once the C-4 simulation space is filled with polyisobutylene, the air scatterers are added to only the polyisobutylene using the same procedure taking care that the air voids do not 
overlap and that they occur only in the polyisobutylene. The FDTD simulation is run on this case, and the far-field data is recorded. The rough surface and the centers of both the polyisobutylene and air scatterers are then redetermined using a random number generator for a total of 50 different cases (discretized at 40 cells per wavelength at $1.5 \mathrm{THz}$ ) as needed for the Monte-Carlo analysis.

The surface length was $10 \mathrm{~cm}$. A monostatic (i.e., transmitter and receiver are co-located) detection regime is assumed for this simulation to more closely match a possible real-world application. The average of these simulation cases is then reported.

\subsection{Impact of the Rough Surface and the Granular Nature of Composition-4 Spectral Signature: No System Noise}

The FDTD simulations were run with an angle of incidence of $-37^{\circ}$. The FDTD results include far-field data at all receive angles and frequencies. The FDTD RCS data is averaged over a 5 degree window centered at a receive angle of $-37^{\circ}$ (relative to the normal) to approximate a receiving antenna with a finite width main lobe. When 20 or 50 surface samples were used for the average field, similar performance for extracting the peaks was found as demonstrated in Chapter 5. Therefore, only data for 50 samples is presented.

The results for cases of a rough and smooth surface with both small and large grain sizes are similar. Therefore, only results for a rough surface and smooth 


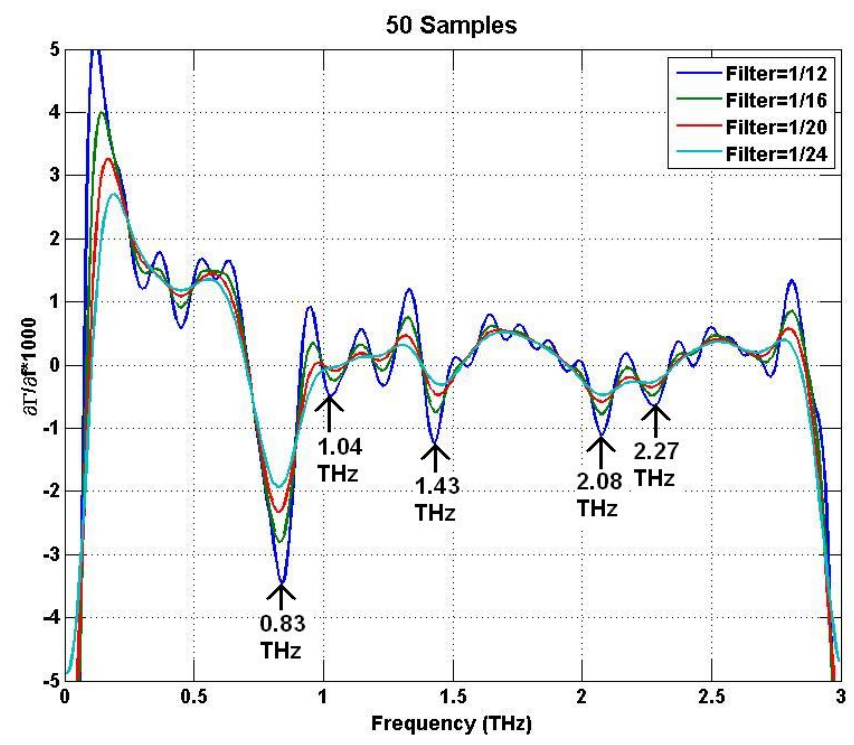

Figure 7.1: Derivative of smoothed data sampled at $-37^{\circ}$ from a background of C4 with a 36 grit surface and a grain size of $100 \mu \mathrm{m}$. Fifty samples were averaged. The black arrows identify the peaks in Table 5.2 to within $5 \%$.

surface with a small grain size are presented.

For all the surface and grain size combinations, the sampled backscattered data is smoothed using a gaussian shaped filter with a standard deviation swept from $1 / 12^{\text {th }}$ to $1 / 24^{\text {th }}$ the sample space. The derivative of the smoothed data for a rough surface and small grain size is presented in Figure 7.1. The derivative of the smoothed data for a rough surface and small grain size is presented in Figure 7.2.

Figure 7.1 shows that five of the six peaks can be extracted from the case run with 50 averaged surfaces. The peaks that are detected are within $5 \%$ of the expected value. The number of false peaks is reduced with more filtering, at the 


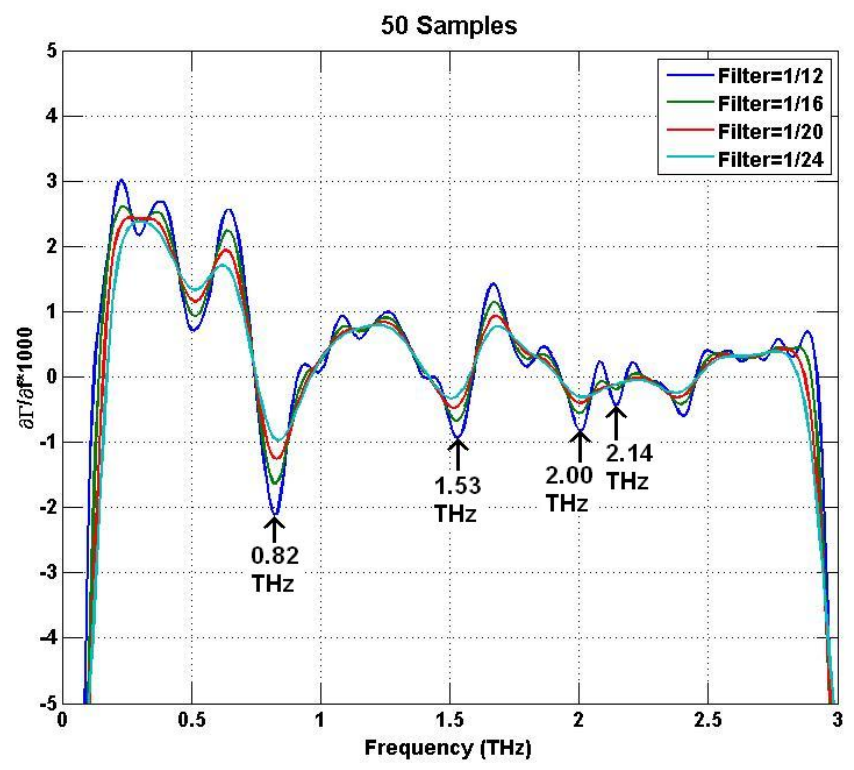

Figure 7.2: Derivative of smoothed data sampled at $-37^{\circ}$ from a background of C4 with a 80 grit surface and a grain size of $100 \mu \mathrm{m}$. Fifty samples were averaged. The black arrows identify the peaks in Table 5.2 to within $5 \%$.

cost of lost Lorentz poles. The same was true for the 36 grit surface with a 300 $\mu \mathrm{m}$ grain.

Figure 7.2 shows that four of the six peaks can be extracted from the case run with 50 averaged surfaces. The peaks that are detected are within $5 \%$ of the expected value. The number of false peaks is reduced with more filtering, at the cost of lost Lorentz poles. The same was true for the 80 grit surface with a 300 $\mu \mathrm{m}$ grain.

Typically, for the case of 36 grit surface interface and 80 grit surface interface using $100 \mu \mathrm{m}$ or $300 \mu \mathrm{m}$ sized C-4 grains, it is possible to extract most of the Lorentz poles. Although not shown, the peaks were also visible with as few as 
20 samples as was demonstrated in Chapter 5. The next section will explore the addition of noise to the system.

\subsection{Impact of the Rough Surface and the Granular Nature of Composition-4 Spectral Signature: With System Noise}

Until this point, the focus has been on extracting the spectral peaks as measured from a sample with a granular nature in a simulator, which has infinite Signalto-Noise Ratio (SNR). A real detection system will have noise, and that must be considered in this analysis. For the 50 cases run for the Monte-Carlo analysis used in the prior section, varying amounts of random frequency domain noise will be added to the data from each simulation case to give an SNR of $0 \mathrm{~dB}, 10 \mathrm{~dB}$, and $20 \mathrm{~dB}$. These cases with noise will then be averaged over 50 surfaces to see what impact the noise may have on the detection of the peaks. The filter standard deviation is set to $1 / 16^{\text {th }}$ the space to maximize the number of actual peaks seen, while minimizing the false peaks. Data for 20 samples is not presented, but the noise performance was the same regardless of whether 20 or 50 surfaces were used to obtain the average field. Therefore, only data using 50 surfaces is presented.

The results for a 36 grit surface with $100 \mu \mathrm{m}$ grain size material with $-37^{\circ}$ angle of incidence with noise is shown in Figure 7.3.

As shown in Figure 7.3, when enough noise is added to the simulated FDTD data to attain an SNR of $20 \mathrm{~dB}$, there is virtually no change in the extracted 


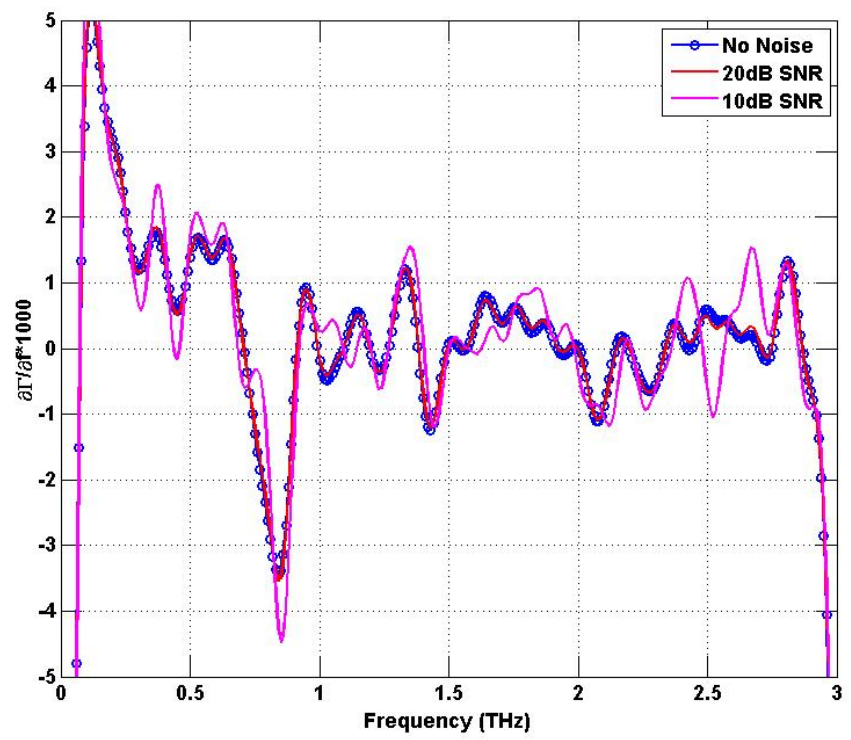

Figure 7.3: Derivative of smoothed data, with various SNR, sampled at $-37^{\circ}$ from a background of C-4 with a 36 grit interface and a grain size of $100 \mu \mathrm{m}$. Fifty samples were averaged.

Lorentz poles. When enough noise is added to attain an SNR of $10 \mathrm{~dB}$, the spectral signature is beginning to be distorted, but the extracted peaks are still visible. When enough noise is added to attain an SNR of $0 \mathrm{~dB}$, the spectral signature is totally changed with the peaks shifting around in frequency and many false peaks appearing. Therefore, an SNR of $10 \mathrm{~dB}$ should be maintained for reliable detection The same $10 \mathrm{~dB}$ SNR limit was observed for the larger grain and for the smoother surface, and data are not presented for these cases. 


\subsection{Other Angles of Incidence}

Other angles of incidence were simulated and the data processed. In general, the same lack of dependence on angle observed in Chapter 5 is seen here. Up to $-50^{\circ}$ there is sufficient signal strength to extract the spectral signature. By the time $-70^{\circ}$ is reached, the signal power drops off significantly making reliable detection difficult, especially in the presence of system noise.

Since the results for 20 and 50 averages surfaces with a rough surface interface and granular scatterers is similar to the results in Chapter 5, only the results for 50 averaged surfaces will be presented here.

\subsubsection{Rough Surface with Small Grain Size}

The results for a 36 grit surface with $100 \mu \mathrm{m}$ grain is given in Table 7.2 for 50 averaged surfaces. The data show that over angle of incidence, it is still possible to see the actual Lorentz poles. At least five of the six peaks are visible. As for the $-37^{\circ}$ results, less filtering has some false peaks. Increased filtering will reduce the false peaks, but it causes the $1.064 \mathrm{THz}$ peak to disappear and the 1.360 and 1.532 $\mathrm{THz}$ peaks either merge, or the $1.532 \mathrm{THz}$ peaks is lost.

When random noise was added the received signal, it was determined that a $10 \mathrm{~dB}$ SNR was required to ensure reliable detection. This is the same result observed for the $-37^{\circ}$ case and in Chapter 5 . 
Table 7.2: Location (in $\mathrm{THz}$ ) of extracted Lorentz poles for data sampled at $-10^{\circ}$, $-30^{\circ}$, and $-50^{\circ}$ from a background of C-4 with the surface roughness of 36 grit sandpaper and a $100 \mu \mathrm{m}$ grain size. Data reported for peaks within $5 \%$ of the expected value. Red values are within $10 \%$.

\begin{tabular}{|c|c||c|c|c|c|c|c|}
\hline $\begin{array}{c}\text { Angle } \\
\text { of Inci- } \\
\text { dence }\end{array}$ & $\begin{array}{c}\text { Filter } \\
\text { Width }\end{array}$ & $\begin{array}{c}0.808 \\
\text { THz } \\
\text { Peak }\end{array}$ & $\begin{array}{c}1.064 \\
\text { THz } \\
\text { Peak }\end{array}$ & $\begin{array}{c}1.360 \\
\text { THz } \\
\text { Peak }\end{array}$ & $\begin{array}{c}1.532 \\
\text { THz } \\
\text { Peak }\end{array}$ & $\begin{array}{c}1.980 \\
\text { THz } \\
\text { Peak }\end{array}$ & $\begin{array}{c}2.224 \\
\text { THz } \\
\text { Peak }\end{array}$ \\
\hline \hline \multirow{4}{*}{$-10^{\circ}$} & $1 / 12^{\text {th }}$ & 0.76 & 1.16 & 1.37 & 1.48 & 1.94 & 2.23 \\
\cline { 2 - 8 } & $1 / 16^{\text {th }}$ & 0.77 & 1.15 & 1.39 & 1.48 & 1.94 & 2.23 \\
\cline { 2 - 8 } & $1 / 20^{\text {th }}$ & 0.77 & - & - & 1.46 & 1.94 & 2.22 \\
\cline { 2 - 8 } & $1 / 24^{\text {th }}$ & 0.78 & - & - & 1.46 & - & 2.22 \\
\hline \hline \multirow{4}{*}{$-30^{\circ}$} & $1 / 12^{\text {th }}$ & 0.81 & 1.08 & 1.37 & 1.66 & 2.01 & 2.22 \\
\cline { 2 - 8 } & $1 / 16^{t h}$ & 0.81 & - & 1.38 & 1.65 & 2.01 & 2.21 \\
\cline { 2 - 8 } & $1 / 20^{\text {th }}$ & 0.81 & - & 1.38 & 1.65 & 2.01 & 2.20 \\
\cline { 2 - 8 } & $1 / 24^{t h}$ & 0.81 & - & 1.38 & - & 2.01 & - \\
\hline \hline \multirow{3}{*}{$-50^{\circ}$} & $1 / 12^{\text {th }}$ & 0.84 & - & 1.35 & 1.59 & 1.97 & 2.21 \\
\cline { 2 - 8 } & $1 / 16^{\text {th }}$ & 0.84 & - & 1.35 & 1.59 & 1.97 & 2.22 \\
\cline { 2 - 8 } & $1 / 20^{\text {th }}$ & 0.84 & - & 1.35 & - & 1.97 & 2.24 \\
\cline { 2 - 8 } & $1 / 24^{\text {th }}$ & 0.83 & - & - & - & 1.97 & - \\
\hline
\end{tabular}

\subsubsection{Rough Surface with Large Grain Size}

The results for a 36 grit surface with $300 \mu \mathrm{m}$ grain is given in Table 7.3 for 50 averaged surfaces. The data shows that over angle of incidence it is still possible to see the actual Lorentz poles. At least five of the six peaks are visible for limited filtering. As for the $-37^{\circ}$ results, less filtering has some false peaks. Increased filtering will reduce the false peaks, but it causes the $1.064 \mathrm{THz}$ peak to disappear and the 1.360 and $1.532 \mathrm{THz}$ peaks either merge, or one of them is lost.

When random noise was added the received signal, it was determined that 
Table 7.3: Location (in $\mathrm{THz}$ ) of extracted Lorentz poles for data sampled at $-10^{\circ}$, $-30^{\circ}$, and $-50^{\circ}$ from a background of $\mathrm{C}-4$ with the surface roughness of 36 grit sandpaper and a $300 \mu \mathrm{m}$ grain size. Data reported for peaks within $5 \%$ of the expected value.

\begin{tabular}{|c|c||c|c|c|c|c|c|}
\hline $\begin{array}{c}\text { Number } \\
\text { of Sur- } \\
\text { faces }\end{array}$ & $\begin{array}{c}\text { Filter } \\
\text { Width }\end{array}$ & $\begin{array}{c}0.808 \\
\text { THz } \\
\text { Peak }\end{array}$ & $\begin{array}{c}1.064 \\
\text { THz } \\
\text { Peak }\end{array}$ & $\begin{array}{c}1.360 \\
\text { THz } \\
\text { Peak }\end{array}$ & $\begin{array}{c}1.532 \\
\text { THz } \\
\text { Peak }\end{array}$ & $\begin{array}{c}1.980 \\
\text { THz } \\
\text { Peak }\end{array}$ & $\begin{array}{c}2.224 \\
\text { THz } \\
\text { Peak }\end{array}$ \\
\hline \hline \multirow{4}{*}{$-10^{\circ}$} & $1 / 12^{\text {th }}$ & 0.79 & - & 1.39 & 1.58 & 1.88 & 2.19 \\
\cline { 2 - 8 } & $1 / 16^{\text {th }}$ & 0.80 & - & - & 1.57 & 1.90 & 2.20 \\
\cline { 2 - 8 } & $1 / 20^{\text {th }}$ & 0.80 & - & - & 1.56 & 1.91 & 2.20 \\
\cline { 2 - 8 } & $1 / 24^{\text {th }}$ & 0.81 & - & - & 1.55 & 1.92 & 2.20 \\
\hline \hline \multirow{4}{*}{$-30^{\circ}$} & $1 / 12^{\text {th }}$ & 0.80 & - & 1.39 & 1.58 & 2.01 & 2.33 \\
\cline { 2 - 8 } & $1 / 16^{t h}$ & 0.80 & - & 1.39 & 1.59 & 2.01 & 2.33 \\
\cline { 2 - 8 } & $1 / 20^{\text {th }}$ & 0.80 & - & 1.39 & - & 2.01 & - \\
\cline { 2 - 8 } & $1 / 24^{\text {th }}$ & 0.80 & - & 1.39 & - & 2.01 & - \\
\hline \hline \multirow{4}{*}{$-50^{\circ}$} & $1 / 12^{\text {th }}$ & 0.84 & 1.08 & 1.38 & 1.49 & 1.90 & 2.33 \\
\cline { 2 - 8 } & $1 / 16^{\text {th }}$ & 0.84 & - & 1.38 & 1.49 & 1.91 & 2.33 \\
\cline { 2 - 8 } & $1 / 20^{\text {th }}$ & 0.84 & - & - & 1.48 & 1.91 & 2.32 \\
\cline { 2 - 7 } & $1 / 24^{\text {th }}$ & 0.84 & - & - & 1.48 & 1.91 & - \\
\hline
\end{tabular}

a $10 \mathrm{~dB}$ SNR was required to ensure reliable detection. This is the same result observed for the $-37^{\circ}$ case and in Chapter 5 .

\subsubsection{Smoother Surface with Small Grain Size}

The results for a 80 grit surface with $100 \mu \mathrm{m}$ grain is given in Table 7.4 for 50 averaged surfaces. The data show that over angle of incidence, it is still possible to see the actual Lorentz poles. At least four of the six peaks are visible. As for the $-37^{\circ}$ results, less filtering has some false peaks. Increased filtering will reduce 
Table 7.4: Location (in $\mathrm{THz}$ ) of extracted Lorentz poles for data sampled at $-10^{\circ}$, $-30^{\circ}$, and $-50^{\circ}$ from a background of $\mathrm{C}-4$ with the surface roughness of 80 grit sandpaper and a $100 \mu \mathrm{m}$ grain size. Data reported for peaks within $5 \%$ of the expected value. Red values are within $10 \%$.

\begin{tabular}{|c|c||c|c|c|c|c|c|}
\hline $\begin{array}{c}\text { Angle } \\
\text { of Inci- } \\
\text { dence }\end{array}$ & $\begin{array}{c}\text { Filter } \\
\text { Width }\end{array}$ & $\begin{array}{c}0.808 \\
\text { THz } \\
\text { Peak }\end{array}$ & $\begin{array}{c}1.064 \\
\text { THz } \\
\text { Peak }\end{array}$ & $\begin{array}{c}1.360 \\
\text { THz } \\
\text { Peak }\end{array}$ & $\begin{array}{c}1.532 \\
\text { THz } \\
\text { Peak }\end{array}$ & $\begin{array}{c}1.980 \\
\text { THz } \\
\text { Peak }\end{array}$ & $\begin{array}{c}2.224 \\
\text { THz } \\
\text { Peak }\end{array}$ \\
\hline \hline \multirow{4}{*}{$-10^{\circ}$} & $1 / 12^{\text {th }}$ & 0.85 & 1.17 & 1.34 & 1.47 & 2.08 & 2.21 \\
\cline { 2 - 8 } & $1 / 16^{\text {th }}$ & 0.85 & 1.16 & 1.34 & 1.47 & 2.08 & 2.21 \\
\cline { 2 - 8 } & $1 / 20^{\text {th }}$ & 0.85 & - & - & 1.45 & - & 2.21 \\
\cline { 2 - 8 } & $1 / 24^{\text {th }}$ & 0.85 & - & - & 1.45 & - & 2.21 \\
\hline \hline \multirow{4}{*}{$-30^{\circ}$} & $1 / 12^{\text {th }}$ & 0.82 & - & 1.38 & 1.60 & 1.98 & 2.29 \\
\cline { 2 - 8 } & $1 / 16^{t h}$ & 0.82 & - & 1.38 & 1.60 & 1.99 & 2.29 \\
\cline { 2 - 8 } & $1 / 20^{\text {th }}$ & 0.82 & - & 1.38 & 1.59 & 2.00 & 2.29 \\
\cline { 2 - 8 } & $1 / 24^{t h}$ & 0.82 & - & 1.38 & - & 2.00 & 2.29 \\
\hline \hline \multirow{3}{*}{$-50^{\circ}$} & $1 / 12^{\text {th }}$ & 0.82 & - & 1.37 & - & 2.06 & 2.32 \\
\cline { 2 - 8 } & $1 / 16^{\text {th }}$ & 0.82 & - & 1.37 & - & 2.06 & 2.32 \\
\cline { 2 - 8 } & $1 / 20^{\text {th }}$ & 0.82 & - & - & - & 2.06 & 2.31 \\
\cline { 2 - 8 } & $1 / 24^{\text {th }}$ & 0.82 & - & - & - & 2.06 & - \\
\hline
\end{tabular}

the false peaks, but it causes the $1.064 \mathrm{THz}$ peak to disappear and the 1.360 and 1.532 THz peaks either merge, or the $1.532 \mathrm{THz}$ peaks is lost.

When random noise was added to the received signal, it was determined that a $10 \mathrm{~dB}$ SNR was required to ensure reliable detection. This is the same result observed for the $-37^{\circ}$ case and in Chapter 5 . 
Table 7.5: Location (in $\mathrm{THz}$ ) of extracted Lorentz poles for data sampled at $-10^{\circ}$, $-30^{\circ}$, and $-50^{\circ}$ from a background of C- 4 with the surface roughness of 80 grit sandpaper and a $100 \mu \mathrm{m}$ grain size. Data reported for peaks within $5 \%$ of the expected value. Red values are within $10 \%$.

\begin{tabular}{|c|c||c|c|c|c|c|c|}
\hline $\begin{array}{c}\text { Angle } \\
\text { of Inci- } \\
\text { dence }\end{array}$ & $\begin{array}{c}\text { Filter } \\
\text { Width }\end{array}$ & $\begin{array}{c}0.808 \\
\text { THz } \\
\text { Peak }\end{array}$ & $\begin{array}{c}1.064 \\
\text { THz } \\
\text { Peak }\end{array}$ & $\begin{array}{c}1.360 \\
\text { THz } \\
\text { Peak }\end{array}$ & $\begin{array}{c}1.532 \\
\text { THz } \\
\text { Peak }\end{array}$ & $\begin{array}{c}1.980 \\
\text { THz } \\
\text { Peak }\end{array}$ & $\begin{array}{c}2.224 \\
\text { THz } \\
\text { Peak }\end{array}$ \\
\hline \hline \multirow{4}{*}{$-10^{\circ}$} & $1 / 12^{\text {th }}$ & 0.83 & - & 1.37 & 1.50 & 1.97 & 2.28 \\
\cline { 2 - 8 } & $1 / 16^{\text {th }}$ & 0.83 & - & 1.37 & 1.50 & 1.97 & 2.28 \\
\cline { 2 - 8 } & $1 / 20^{\text {th }}$ & 0.83 & - & - & 1.49 & 1.98 & 2.28 \\
\cline { 2 - 8 } & $1 / 24^{\text {th }}$ & 0.83 & - & - & 1.48 & 1.99 & 2.28 \\
\hline \hline \multirow{4}{*}{$-30^{\circ}$} & $1 / 12^{\text {th }}$ & 0.82 & - & 1.38 & 1.57 & 2.00 & 2.31 \\
\cline { 2 - 8 } & $1 / 16^{t h}$ & 0.82 & - & 1.38 & 1.57 & 2.00 & 2.31 \\
\cline { 2 - 8 } & $1 / 20^{\text {th }}$ & 0.82 & - & 1.38 & - & 2.00 & - \\
\cline { 2 - 8 } & $1 / 24^{\text {th }}$ & 0.82 & - & 1.38 & - & 2.01 & - \\
\hline \hline \multirow{3}{*}{$-50^{\circ}$} & $1 / 12^{\text {th }}$ & 0.81 & 1.10 & 1.49 & 1.57 & 1.96 & 2.29 \\
\cline { 2 - 8 } & $1 / 16^{\text {th }}$ & 0.81 & 1.10 & - & 1.56 & 1.97 & 2.28 \\
\cline { 2 - 8 } & $1 / 20^{\text {th }}$ & 0.81 & 1.10 & - & 1.52 & 1.98 & 2.27 \\
\cline { 2 - 8 } & $1 / 24^{\text {th }}$ & 0.81 & - & - & 1.52 & 1.98 & 2.26 \\
\hline
\end{tabular}

\subsubsection{Smoother Surface with Larger Grain Size}

The results for a 80 grit surface with $300 \mu \mathrm{m}$ grain is given in Table 7.5 for 50 averaged surfaces. The data show that over angle of incidence, it is still possible to see the actual Lorentz poles. At least five of the six peaks are visible. As for the $-37^{\circ}$ results, less filtering has some false peaks. Increased filtering will reduce the false peaks, but it causes the $1.064 \mathrm{THz}$ peak to disappear and the 1.360 and $1.532 \mathrm{THz}$ peaks either merge, or the $1.532 \mathrm{THz}$ peaks is lost.

When random noise was added the received signal, it was determined that 
a $10 \mathrm{~dB}$ SNR was required to ensure reliable detection. This is the same result observed for the $-37^{\circ}$ case and in Chapter 5 .

\subsection{Conclusion}

This chapter looked at the combined effect of rough surface granular scattering on the extracted spectral signature of C-4. It is possible to extract most of the Lorentz poles with a minimum of false peaks if 20 samples were averaged. The $1.064 \mathrm{THz}$ peak was most often lost in this analysis because of the increased filtering required to eliminate the false peaks.

When noise was added to the simulated data, the spectral signature was little changed if a $10 \mathrm{~dB}$ SNR was maintained. Therefore, as with the case of a rough surface only, a 10dB SNR should be maintained.

While the $1.064 \mathrm{THz}$ peak was frequently lost, it is encouraging that five of the peaks were generally visible if the back scattered detection angle was kept within $-50^{\circ}$ of the normal. This will likely provide enough information for reliable detection of C-4. 


\section{Chapter 8}

\section{Conclusion and Future Work}

\subsection{Conclusion}

Terahertz spectroscopy is a promising field for the detection of explosives and other illicit materials. The detection of illicit materials is based on the unique spectral signature of each material. However, the granular nature of the explosives and illicit materials may mask the unique spectral signature. In addition to the granular nature, the interface of the material is likely to be rough at terahertz frequencies which may also mask the unique spectral signature. To become a viable detection method at any security checkpoint, the effect of the rough surface and granular scattering must be understood.

The impact the rough surface and granular scattering has on the spectral signature was explored in this work. It began with a review of basic electromagnetic wave reflections and scattering. The numerical modeling technique, the Finite-Difference Time-Domain (FDTD) method was introduced and tested 
for accuracy against published results and results measured at the University of Maryland Baltimore County and in the Northwest Electromagnetics and Acoustics Research Laboratory.

With the FDTD model validated, simulations were performed to understand how the rough surface may mask the spectral signature of the common explosive Composition-4 (C-4). When reflected energy was sampled in the backscatter direction from multiple rough surfaces, the spectral signature was not visible by using the derivative technique. However, a filtering method was proposed to remove the "noise" associated with a finite number of rough surface samples to show the spectral signature of C-4 could be reliably extracted with as few as 20 samples over a wide range of receive angles (up to $-50^{\circ}$ measured from the normal) with an SNR of 10dB.

The FDTD model was then used to determine how the granular nature of the C-4 may mask the signature of C-4 if the interface was a smooth surface. While the granular nature of the C-4 allowed for energy to be spread into the backscatter direction, the frequency dependence of the granular scatterers did not allow all of the Lorentz poles to be reliably extracted. However, the small amount of power that was reflected indicated that the scattering would be dominated by the rough surface interface.

Finally, the rough surface and granular nature of the C-4 was combined and run in the FDTD model. This model showed that with similar filtering it was 
possible to extract most of the six Lorentz poles of C-4 with as few as 20 samples over a wide range of receive angle (up to $-50^{\circ}$ measured from the normal) with an SNR of 10dB.

It was shown that it is possible to extract the location of the Lorentz poles to identify the spectral signature of $\mathrm{C}-4$ from the back scattered energy. This work focused only back scattered energy, but the algorithm to extract the Lorentz poles would work equally well if there was any specular scattering (i.e. glinting). While the surfaces studied in this work were extremely rough, it is likely that sufficient energy would be back scattered to allow for the detection of the spectral signature in a mono-static detection regime. Radar systems are a good example of a mono-static detection regime that detects smooth surfaces with little back scattered energy. To deal with the limited amount of back scattered energy, more detectors could be used to increase the amount of energy received.

\subsection{Future Work}

Several areas could be researched to continue this work.

1. Move to a three dimensional simulation for more accurate modeling of the actual material. This will allow simulations of surfaces and structures actually measured (i.e. full modeling of surface topography and internal structure with minimal approximations). 
2. Conformal meshing and adaptive meshing to simulate larger problems with less memory. Conformal meshing would be required for a full three-dimensional simulation.

3. Employ Periodic Boundary Conditions to see if the size of the simulated space can be reduced. It may be possible to create a rough surface (and/or a distribution of granular scatterers) that has un-correlated ends but is periodic. The advantage to this may be the simulation of a smaller surface, but this results could be easily scaled to any length of surface.

4. Simulate grain rotation to understand if the anisotropic nature of the explosive grains would mask the signature. In practice, this is not a short coming since the orientation of the crystals in the explosive would lead to the explosive looking isotropic macroscopically.

5. Adaptive filtering to adjust to power levels and number of samples.

6. Model a source wave with a shape beyond a plane wave. For example, enter in the antenna of a measurement system to model a wave encountered in a real measurement system.

7. Model finite thickness or layered media. 


\section{References}

[1] Matthew C. Beard, Gordon M. Turner, and Charles A. Schmuttenmaer. Terahertz spectroscopy. Journal of Physical Chemistry B, 106(29):7146 $7159,2002$.

[2] Matthew B. Campbell and Edwin J. Heilweil. Non-invasive detection of weapons of mass destruction using thz radiation. Proceedings of SPIE - The International Society for Optical Engineering, 5070:38 - 43, 2003.

[3] Frances T. Docherty, Paul B. Monaghan, Callum J. Mchugh, Duncan Graham, W. Ewen Smith, and Jonathan M. Cooper. Simultaneous multianalyte identification of molecular species involved in terrorism using raman spectroscopy. IEEE Sensors Journal, 5(4):632 - 639, 2005.

[4] John F. Federici, Brian Schulkin, Feng Huang, Dale Gary, Robert Barat, Filipe Oliveira, and David Zimdars. Thz imaging and sensing for security applications - explosives, weapons and drugs. Semiconductor Science and Technology, 20(7):226 - 280, 2005. 
[5] Jeffrey Barber, Daniel E. Hooks, David J. Funk, Richard D. Averitt, Antoinette J. Taylor, and Dmitri Babikov. Temperature-dependent far-infrared spectra of single crystals of high explosives using terahertz time-domain spectroscopy. Journal of Physical Chemistry A, 109(15):3501 - 3505, 2005.

[6] Yunqing Chen, Haibo Liu, Yanqing Deng, Dmitry Veksler, Michael Shur, X.-C. Zhang, Dunja Schauki, Michael J. Fitch, Robert Osiander, Caroline Dodson, and James B. Spicer. Spectroscopic characterization of explosives in the far infrared region. Proceedings of SPIE - The International Society for Optical Engineering, 5411:1 - 8, 2004.

[7] Yunqing Chen, Haibo Liu, Michel J. Fitch, Robert Osiander, James B. Spicer, Michael Shur, and X.-C. Zhang. Thz diffuse reflectance spectra of selected explosives and related compounds. Proceedings of SPIE - The International Society for Optical Engineering, 5790:19 - 24, 2005.

[8] David W. Ball. Field Guide to Spectroscopy. SPIE Press, 2006.

[9] Andrew Burnett, Wenhui Fan, Prashanth Upadhya, John Cunningham, Edmund Linfield, Giles Davies, Howell Edwards, Tasnim Munshi, and Andrew O'Neil. Analysis of drugs of abuse and explosives using terahertz timedomain and raman spectroscopy. Proceedings of SPIE - The International Society for Optical Engineering, 6120:1 - 7, 2006. 
[10] K. Yamamoto, M. Yamaguchi, F. Miyamaru, M. Tani, M. Hangyo, T. Ikeda, A. Matsushita, K. Koide, M. Tatsuno, and Y. Minami. Noninvasive inspection of c-4 explosive in mails by terahertz time-domain spectroscopy. Japanese Journal of Applied Physics, Part 2: Letters, 43(3):414-417, 2004.

[11] William R. Tribe, David A. Newnham, Philip F. Taday, and Michael C. Kemp. Hidden object detection: Security applications of terahertz technology. Proceedings of SPIE - The International Society for Optical Engineering, 5354:168 - 176, 2004.

[12] Y.C. Shen, T. Lo, P.F. Taday, B.E. Cole, W.R. Tribe, and M.C. Kemp. Detection and identification of explosives using terahertz pulsed spectroscopic imaging. Applied Physics Letters, 86(24):1 - 3, 2005.

[13] L.M. Zurk, B. Orlowski, G. Sundberg, D.P. Winebrenner, E.I. Thorsos, and A. Chen. Electromagnetic scattering calculations for terahertz sensing. January 2007.

[14] L.M. Zurk, B. Orlowski, Z. Sundberg, G. Zhou, and A. Chen. Terahertz scattering from a rough granular surface. June 2007.

[15] S.C. Henry, G. Kniffin, S. Schecklman, and L.M. Zurk. Measurement and modeling of rough surface effects on terahertz spectroscopy and imaging. Proceedings of SPIE, 7601. 
[16] R. Appleby and H.B. Wallace. Standoff detection of weapons and contraband in the $100 \mathrm{ghz}$ to $1 \mathrm{thz}$ region. IEEE Transactions on Antennas and Propagation, 55(11):2944 - 2956, 2007.

[17] H. Zhong, A. Redo, Y. Chen, and X.C. Zhang. Thz wave standoff detection of explosive materials. Proceedings of SPIE, 6212, 2006.

[18] M. Chen, J.H. Kim, C. Yang, S. Yin, Z. Liu, and R. Hui. Thz generation in multiple air plasmas. Proceedings of SPIE - The International Society for Optical Engineering, 7056, 2008.

[19] J.E. Reaugh. Checking out the hot spots. Science and Technology Review, pages $19-21,2003$.

[20] Constantine A. Balanis. Advanced Engineering Electromagnetics. John Wiley and Sons, 1989.

[21] K.S. Yee. Numerical solutions of initial boundary value problems involving maxwell's equations in isotropic media. IEEE Transactions on Antennas and Propagation, 14(3):302 - 307, 1966.

[22] Rudolf Meyer. Explosives Third, revised and extended edition. Weinheim, New York:VCH, 1987. 
[23] K. Raha and J.S. Chhabra. Static charge development and impact sensitivity of high explosives. Journal of Hazardous Materials, 34:385-391, 1993. El Sevier Science Publishers BV Amsterdam.

[24] C.B. Skidmore, D.S. Phillips, and N.B. Crane. Microscopical examination of plastic-bonded explosives. Technical report, Los Alamos National Laboratory, Los Alamos, New Mexico 87545, 1991.

[25] F. Huang, B. Shulkin, H. Altan, J. Federeci, D. Gary, R. Barat, D. Zimdarr, M. Chen, and D.B. Tanner. Terahertz study of 1, 3, 5 - trinitro-striazine (rdx) by time domain spectroscopy and ftir. Applied Physics Letters, 83(2477):5535 - 5537, 2003.

[26] R. Oslander, J.A. Miragliotta, Z. Jiang, J. Xu, and X.C. Zhang. Mine field detection and identification using thz spectroscopic imaging. Proceedings of SPIE, 5070, 2003.

[27] M.J. Fitch, D. Schauki, C.A. Kelly, and R. Oslander. Terahertz imaging and spectroscopy for landmine detection. Proceedings of SPIE, 5354, 2004.

[28] D.J. Cook, B. Decker, and M. Allen. Quantative thz spectroscopy of explosive materials. Optical Terahertz Science Technology, 2005. 
[29] A. Bandyopadhyay, A. Seragupta, F. Huang, J.F. Fderici, R.B. Barsat, D.E. Gary, M. Chen, and D.B. Tanner. Impact of grain size dependent scattering on terahertz absorption spectra. Applied Optics, page 2005.

[30] David K. Cheng. Field and Wave Electromagnetics. Addison Wesley, 1989.

[31] David J. Griffiths. Introduction to Electrodynamics. Prentice hall, 1999.

[32] L. Tsang, J.A. Kong, and K.H. Ding. Scattering of Electromagnetic Waves, Volume 1-3. Wiley Publishers., 2000-2001.

[33] David M. Pozar. Microwave Engineering, 3rd Edition. Wiley, New York, 2001.

[34] Allen Taflove and Susan Hagness. Computational Electrodynamics. The Finite-Difference Time-Domain Method. Third Edition. Artech House, 2005.

[35] L.M. Zurk, B. Orlowski, D. Winebrenner, E. Thorsos, M. Leahy-Hoppa, and M. Hayden. Terahertz scattering from granular material. Journal of the Optical Society of America B: Optical Physics, 24(9):2238-2243, 2007.

[36] D. E. Merewether, R. Fisher, and F. W. Smith. On implementing a numeric huygen's source scheme in a finite difference program to illuminate scattering bodies. IEEE Transactions on Nuclear Science, 27(6):1829 - 1830, 1980. 
[37] Gerrit Mur. Absorbing boundary conditions for the finite-difference approximation of the time-domain electromagnetic-field equations. IEEE Transactions on Electromagnetic Compatibility, 23(4):377 - 382, 1981.

[38] Korada Umashankar and Allen Taflove. A novel method to analyze electromagnetic scattering of complex objects. IEEE Transactions on Electromagnetic Compatibility, 24(4):397 - 405, 1982.

[39] J.B. Schneider and K. Abdijalilov. Analytic field propagation tfsf boundary for fdtd problems involving planar interfaces: Pecs, te, and tm. IEEE Transactions on Antennas and Propagation, 54(9):2531 - 2542, 2006.

[40] J.B. Schneider. Plane waves in fdtd simulations and a nearly perfect totalfield/scattered-field boundary. IEEE Transactions on Antennas and Propagation, 52(12):3280 - 3287, 2004.

[41] John B. Schneider and Christopher L. Wagner. Fdtd dispersion revisited: Faster-than-light propagation. IEEE Microwave and Guided Wave Letters, 9(2):54-56, 1999.

[42] K. Abdijalilov and J.B. Schneider. Analytic field propagation tfsf boundary for fdtd problems involving planar interfaces: Lossy material, evanescent fields. IEEE Antennas and Wireless Propagation Letter, 5:454-458, 2006. 
[43] Jean Pierre Berenger. A perfectly matched layer for the absorption of electromagnetic waves. Journal of Computational Physics, 114:185 - 200, 1994.

[44] Z.S. Sacks, D.M. Kingsland, R. Lee, and J.F. Lee. A perfectly matched anisotropic absorber for use as an absorbing boundary condition. IEEE Transactions on Antennas and Propagation, 43:1460-1463, 1995.

[45] S.D. Gedney. An anisotropic perfectly matched layer absorbing media for the truncation of fdtd lattices. IEEE Transactions on Antennas and Propagation, 44:1630-1639, 1996.

[46] J. A. Roden and S. D. Gedney. Convolutional pml (cpml): An efficient fdtd implementation of the cfs-pml for arbitrary media. Microwave Optical Technical Letters, 27:334 - 339, 2000.

[47] Stephen D. Gedney. An anisotropic pml absorbing media for fdtd simulation of fields in lossy dispersive media. Electromagnetics, 16:399-415, 1996.

[48] J. D. Jackson. Classical Electrodynamics. John Wiley and Sons, 1975.

[49] D.J. Robinson and J.B. Schneider. On the use of the geometric mean in fdtd near-to-far-field transformations. IEEE Transactions on Antennas and Propagation, 55(11):3204 - 3211, 2007. 
[50] Frank D. Hastings, John B. Schneider, and Shira L. Broschat. Monte-carlo fdtd technique for rough surface scattering. IEEE Transactions on Antennas and Propagation, 43(11):1183 - 1191, 1995.

[51] D. F. Kelley and R. J. Luebbers. Piecewise linear recursive convolutions for dispersive media using fdtd. IEEE Transactions on Antennas and Propagation, 44:792 - 797, 1996.

[52] Raymond Luebbers and Forrest Hunsberger. Fdtd for nth-order diseprsive media. IEEE Transactions on Antennas and Propagation, 40:1297 - 1301, 1992.

[53] R.J Luebbers, D. Steich, and K. Kunz. Fdtd calculations of scattering from frequency-dependent materials. IEEE Transactions on Antennas and Propagation, 41:1249 - 1257, 1993.

[54] T. Kashiwa and I. Kukai. A treatment by fdtd method of dispersive characteristics associated with electronic polarization. Microwave Optics Technique Letters, 3:203 - 205, 1990.

[55] R.M. Joseph, S. C. Hagness, and A. Taflove. Direct time integration of maxwell's equations in linear dispersive media with absorption for scattering and propagation of femtosecond electromagnetic pulses. Optics Letters, 16:1412 - 1414, 1991. 
[56] M. Okoniewski, M. Mrozowski, and M.A. Stuchly. Simple treatment of multi-term disperson in fdtd. IEEE Microwave Guided Wave Letters, 7:121 $-123,1997$.

[57] T. O. Korner and W. Fichtner. Dispersive media in fdtd - comparison of recursive convolution and auxiliary differential equation methods. Technical Proceedings of the 1998 International Conference on Modeling and Simulation of Microsystems, pages 339 - 343, 1998.

[58] L.M. Hayden, A.M. Sinyukov, M.R. Leahy, J. French, P. Lindahl, W.N. Herman, Tweig R.J., and M. He. New materials for optical rectification and electrooptic sampling of ultrashort pulses in the terahertz regime. Journal of Polymer Science, 41:2492 - 2005, 2003.

[59] A.M. Sinyukov and L.M. Hayden. Efficient electrooptic polymers for thz applications. Journal of Physical Chemistry, 108:8515 - 8522, 2004.

[60] P. Pedersen and A. Grebe. Measurement of rough surface scattering using time delay spectrometry. Proceedings of the IEEE Ultrasonic Symposium, 1:827-831, 1997.

[61] W. Makinde, N. Favretto-Cristini, and E. de Bazelaire. Numerical modelling of interface scattering of seismic wavefield from a random rough interface 
in an acoustic medium: Comparison between $2 \mathrm{~d}$ and $3 \mathrm{~d}$ cases. Geophysical Prospecting, 53(3):373 - 397, 2005.

[62] C. Walter and C. Mitterer. 3d versus 2d finite element simulation of the effect of surface roughness on nanoindentation of hard coatings. Surface and Coatings Technology, 203(20-21):3286 - 3290, 2009.

[63] H. Zhong, A. Redo-Sanchez, and X.C. Zhang. Identification and classification of chemicals using terahertz reflective spectroscopic focal plane imaging system. Optics Express, 14(20):9130-9141, 2006.

[64] B.P. Bogert, M.J.R. Healy, and J.W. Tukey. The quefrency alanysis of time series for echoes: Cepstrum, psuedo autocovariance, cross-cepstrum and saphe cracking. Proc. of the Symposium on Time Series Analysis, 15:209243, 1963.

[65] G. Sundberg, L.M. Zurk, S. Schecklman, and S. Henry. Modeling rough surface and granular scattering at terahertz frequencies using the finitedifference time-domain method. IEEE Transactions on Geoscience and Remote Sensing, accepted for publication July, 2010.

[66] L. Borne and A. Beaucamp. Effects of crystal internal defects on projectile impact initiation. 12th International Detonation Symposium, San Diego, CA 11-16 August, 2002. 
[67] Explosives. Technical report, United States Naval Acadamy, 2005. 


\section{Appendix A}

\section{Glossary}

- ABC: Absorbing Boundary Condition. A grid termination for FDTD simulations that reduce spurious reflection when the EM wave interacts with the simulation space boundary.

- AFP: Analytic Field Propagation. An accurate method of implementing a TFSF simulation to introduce a plane wave into an FDTD simulation space.

- B: Magnetic Flux Density in Weber per square meter.

- CPLM: Convolutional PML. A class of PML.

- D: Electric Flux Density in Coulombs per square meter.

- Dispersive Media: A material which has a permittivity or permeability which changes with frequency.

- Effective Dielectric Constant $\left(\epsilon_{r}\right)$ : The relative permittivity of a material.

- Epsilon $(\epsilon)$ : The permittivity of a material defined as $\epsilon_{r} \times \epsilon_{0}$. 
- Epsilon Not $\left(\epsilon_{0}\right)$ : The permittivity of a free space. Approximately $8.854 \times 10^{-12}$ $\mathrm{F} / \mathrm{m}$.

- E: Electric Field Intensity in Volts per meter.

- EM: Electromagnetic

- Eta $(\eta)$ : The intrinsic impedance of a media defined as the square root of $\mu$ divided by $\epsilon$.

- Explosive: a substance or a device that produces a volume of rapidly expanding gas that exerts sudden pressure on its surroundings[67].

- Example: Chemical Explosive Nitroglycerin 4C-4H5(ONO2)3 12CO2 $+10 \mathrm{H} 2 \mathrm{O}+6 \mathrm{~N} 2+\mathrm{O} 2$ Ignition will produce products that are all gases. The 4 moles of compound will yield 29 moles of gas, this is why there is a large pressure wave produced[67].

- Fractional Volume: Volume of interest/volume of space, for example: a cube with sides 1 meter in length has a volume of $1 m^{3}$ filled with 3 spheres of 0.1 radius, volume of spheres is $3 \times \frac{4 \times \pi \times \text { radius }^{3}}{3}=0.0126$. The fractional volume is $\frac{0.0126}{1}=0.0126$. Fractional volume is used to describe the amount of particles filling in our space.

- H: Magnetic Field Intensity in Ampere per meter. 
- J: Electric Current Density in Ampere per square meter.

- M: Magnetic Current Density.

- Monostatic: Remote sensing regime where the source and detector are colocated.

- $\mathrm{Mu} \operatorname{Not}\left(\mu_{0}\right)$ : The permeability of a free space. $4 \pi \times 10^{-7} \mathrm{H} / \mathrm{m}$.

- NTFF: Near-to-Far-Field transformation. A method of transforming the near-field data of an FDTD simulation to the far-field for calculation of RCS and antenna patterns.

- Parallel Polarization $\left(\mathrm{TE}_{z}\right)$ : The plane of an incident wave when the electric field is parallel to the plane in incidence.

- Perpendicular Polarization $\left(\mathrm{TM}_{z}\right)$ : The plane of an incident wave when the electric field is perpendicular to the plane in incidence.

- Permittivity $(\mu)$ : The permittivity of a material defined as $\mu_{r} \times \mu_{0} . \mu_{r}$ is called the relative permeability of the material

- PML: Perfectly Matched Layer. A class of Absorbing Boundary Condition. The most popular and effective grid termination technique.

- QCA: Quasi-Crystalline Approximation. An analytic technique to calculate a frequency dependent effective dielectric constant to account for granular 
scattering in a medium.

- RCS: Radar Cross Section. The ratio of the scattered power to the incident power. Also called Bistatic Scattering Coefficient.

- Rho $(\rho)$. Electric charge in Coulombs.

- Scattering Regimes

- Rayleigh: particle size $<$ wavelength

- Mie: particle size $\approx$ wavelength

- Optics: particle size $>$ wavelength

- Sigma $(\sigma)$ : The conductivity of a material in Siemens per meter.

- Sigma Star $\left(\sigma^{*}\right)$ : The equivalent magnetic conductivity of a material.

- SNR: Signal-to-Noise Ratio. The level of the signal compared to the noise of a measurement system.

- THz: Terahertz. $1 \times 10^{12}$ hertz.

- THz-TDS: Terahertz-Time Domain Spectroscopy. Spectroscopy performed using a small duration time pulse. Frequency data obtained by taking the Fourier Transform of the time-domain data.

- UPML: Uniaxial PML. A class of PML. 